\title{
Effective finiteness theorems for decomposable forms of given discriminant
}

by

\author{
J. H. Evertse* (Leiden) and K. GYŐRY** (Debrecen)
}

Introduction. Let $K$ be an algebraic number field, $S$ a finite set of prime ideals in $K$ and $O_{S}$ the ring of $S$-integers in $K$. Two binary forms $F(X, Y), G(X, Y) \in O_{S}[X, Y]$ are called equivalent if there is a matrix $\left(\begin{array}{ll}\alpha & \beta \\ \gamma & \delta\end{array}\right) \in \mathrm{GL}\left(2, O_{S}\right)$ such that $G(X, Y)=F(\alpha X+\beta Y, \gamma X+\delta Y)$. In 1972, Birch and Merriman [1] proved that for given integer $r \geq 3$, there are only finitely many equivalence classes of binary forms $F \in O_{S}[X, Y]$ of degree $r$ whose discriminant $D(F)$ belongs to the group of $S$-units $O_{S}^{*}$. The proof in [1] is ineffective in the sense that it does not provide an algorithm to find a full set of representatives for these equivalence classes. In a series of papers, Győry [8], [9], [12] obtained effective finiteness results for monic polynomials with coefficients in $O_{S}$ and with given non-zero discriminant; for binary forms $F$ with $F(1,0)=1$ these results imply an effective version of Birch and Merriman's theorem. In our recent paper [6] we made the result of Birch and Merriman effective in full generality, without any restriction on $F$.

The purpose of the present paper is to extend our results from [6] on binary forms to decomposable forms in $n \geq 2$ variables. The general result over algebraic number fields is stated in Section 2; here we restrict ourselves to the case of the field of rationals $\mathbb{Q}$. Let $\left\{p_{1}, \ldots, p_{s}\right\}(s \geq 0)$ be a finite set of primes and consider the ring $R=\mathbb{Z}\left[\left(p_{1} \ldots p_{s}\right)^{-1}\right]$. A polynomial $F(\mathbf{X}) \in$ $R\left[X_{1}, \ldots, X_{n}\right]$ is called a decomposable form if it can be factored as $F(\mathbf{X})=$ $\lambda l_{1}(\mathbf{X})^{k_{1}} \ldots l_{t}(\mathbf{X})^{k_{t}}$ where $\lambda \in \mathbb{Q}^{*}, l_{1}, \ldots, l_{t}$ are pairwise non-proportional homogeneous linear polynomials with coefficients in some algebraic number field $L$ and $k_{1}, \ldots, k_{t}$ are positive integers with $k_{1}+\ldots+k_{t}=\operatorname{deg}(F)$.

* The research of the first author has been made possible by a fellowship of the Royal Netherlands Academy of Arts and Sciences.

** The research of the second author has been supported in part by Grant 273 from the Hungarian National Foundation for Scientific Research. 
Let $\mathcal{I}(F)$ be the collection of $L$-linearly independent subsets $\left\{l_{i_{1}}, \ldots, l_{i_{n}}\right\}$ ( $n=$ number of variables of $F$ ) of $\left\{l_{1}, \ldots, l_{t}\right\}$. We denote the coefficient determinant of $\left\{l_{i_{1}}, \ldots, l_{i_{n}}\right\} \in \mathcal{I}(F)$ by $\operatorname{det}\left(l_{i_{1}}, \ldots, l_{i_{n}}\right)$. Further, by $\widehat{R}$ we denote the integral closure of $R$ in $L$. Denote by $(a)$ the $\widehat{R}$-ideal generated by $a$, and by $\left(l_{i}\right)$ the $\widehat{R}$-ideal generated by the coefficients of $l_{i}$ for $i=1, \ldots, t$. Assume that $\mathcal{I}(F) \neq \emptyset$. Then there is a positive rational integer $D=D_{R}(F)$, composed of prime numbers outside $\left\{p_{1}, \ldots, p_{s}\right\}$, such that

$$
(D)=\prod_{\mathcal{I}(F)}\left\{\frac{\operatorname{det}\left(l_{i_{1}}, \ldots, l_{i_{n}}\right)}{\left(l_{i_{1}}\right) \ldots\left(l_{i_{n}}\right)}\right\}^{2},
$$

where the product is taken over all sets $\left\{l_{i_{1}}, \ldots, l_{i_{n}}\right\}$ in $\mathcal{I}(F)$; further, the integer $D$ does not depend on the choice of $l_{1}, \ldots, l_{t}$ (cf. Section 3 ) and $D_{R}(\mu F)=D_{R}(F)$ for all $\mu \in \mathbb{Q}^{*}$. The integer $D_{R}(F)$ is called the $R$ discriminant of $F$. If $\mathcal{I}(F)=\emptyset$ then we put $D_{R}(F)=0$. For instance, if $F$ is a binary form with relatively prime coefficients in $\mathbb{Z}$, then $D_{\mathbb{Z}}(F)$ is just the absolute value of the discriminant $D(F)$ of $F$. Two decomposable forms $F(\mathbf{X}), G(\mathbf{X}) \in R\left[X_{1}, \ldots, X_{n}\right]$ are called $R$-equivalent if there is a matrix $U \in$ $\mathrm{GL}(n, R)$ with $G(\mathbf{X})=F(U \mathbf{X})$. Two $R$-equivalent decomposable forms have the same $R$-discriminant (cf. Section 1$)$. The height of a rational number $a / b$ with $a, b \in \mathbb{Z}, \operatorname{gcd}(a, b)=1$ is defined by $h(a / b)=\max (|a|,|b|)$; the height $h(F)$ of a polynomial $F$ with coefficients in $\mathbb{Q}$ is defined as the maximum of the heights of the coefficients of $F$. We have

Theorem 1. Let $F(\mathbf{X}) \in R\left[X_{1}, \ldots, X_{n}\right]$ be a decomposable form of degree $r$ with relatively prime coefficients and with $D_{R}(F)=D \neq 0$. Then $F$ is $R$-equivalent to a decomposable form $G$ with $h(G) \leq C$, where $C$ is an effectively computable number depending only on $n, r, D, s$ and $p_{1}, \ldots, p_{s}$.

We remark that Theorem 1 implies, in an effective way, that there are only finitely many $R$-equivalence classes of decomposable forms in $R\left[X_{1}, \ldots, X_{n}\right]$ with relatively prime coefficients, with given degree and given non-zero $R$-discriminant.

For $n=2$ and $R=\mathbb{Z}$ (when $s=0$ ), Theorem 1 gives (in a less explicit form) Theorem 1 of [6] on binary forms with given discriminant.

We shall get Theorem 1 as a special case of a more general result on decomposable forms on $O_{S}$-modules, where $O_{S}$ is the ring of $S$-integers of an algebraic number field (cf. Section 2, Corollary 4). The proof of this general result uses an effective result of Györy ([10], Lemma 6) on the $S$-unit equation in two variables; so the proof of our result ultimately goes back to Baker's theory on linear forms in logarithms and its $p$-adic analogue.

As an application of our general results on decomposable forms, we deduce (cf. Section 2, Corollary 6) an effective finiteness result for finitely 
generated $O_{S}$-modules with given discriminant. Our results on decomposable forms can also be applied to the study of decomposable form equations of the form

$$
F(\mathbf{x})=a \quad \text { in } \mathbf{x}=\left(x_{1}, \ldots, x_{n}\right) \in R^{n},
$$

where $F(\mathbf{X})$ is as in Theorem 1 and $a \in R \backslash\{0\}$. For instance, if one can prove that the set of solutions of $(*)$ has a special structure provided that $D_{R}(F)$ is sufficiently large, then it follows that there are only finitely many $R$-equivalence classes of decomposable forms $F$ for which the set of solutions of $(*)$ does not have that special structure. A result of this type will be published in a forthcoming paper of the first author [3], which extends to the case $n \geq 2$ Theorem 2(i) of [5] obtained for $n=2$. Another possible application concerns effective results on equation $(*)$. For a certain class of decomposable forms which is invariant under linear transformations of $F$ and which includes binary forms, discriminant forms, index forms and certain special norm forms (cf. [11], [4]) it is possible to give an effectively computable number $C_{F}^{\prime}$ depending only on $n, r, s, p_{1}, \ldots, p_{s}, h(F)$ and $a$, such that $\max _{i} h\left(x_{i}\right) \leq C_{F}^{\prime}$ for every solution $\mathbf{x}=\left(x_{1}, \ldots, x_{n}\right)$ of $(*)$. It might be possible to improve this bound in certain cases, by first looking for a matrix $U \in \mathrm{GL}(n, R)$ such that $G(\mathbf{X})=F(U \mathbf{X})$ has height $\leq C$, then computing the upper bound $C_{G}^{\prime}$ for the heights of the solutions of $(*)$ with $F$ replaced by $G$ and finally deriving an upper bound for the heights of the solutions $\mathbf{x}$ of $(*)$ by estimating from above the heights of the entries of $U^{-1}$. Probably we shall publish a paper about these effective results.

In Section 1, we introduce some general notions about decomposable forms which will be needed in the later sections. In Section 2, we state our effective results about decomposable forms on $O_{S}$-modules. The remaining sections will be devoted to the proofs of these results.

1. General facts on decomposable forms. Let $K$ be a field and $V$ a finite-dimensional $K$-vector space. A decomposable form on $V$ is a function $F: V \rightarrow K$ with the following property: there are an extension $L / K$, a positive integer $r$ and $K$-linear functions $l_{i}: V \rightarrow L(i=1, \ldots, r)$ such that

$$
F(\mathbf{x})=l_{1}(\mathbf{x}) \ldots l_{r}(\mathbf{x}) \quad \text { for all } \mathbf{x} \in V .
$$

We call $\left(l_{1}, \ldots, l_{r}\right)$ a factorization of $F$ in $L$. If $K$ is infinite then $r$ is uniquely determined by $F$; in this case $r$ is called the degree of $F$. The smallest extension $L$ of $K$ in which $F$ has a factorization is called the splitting field of $F$ over $K$; it is a finite, normal extension of $K$. The rank of $F$ is defined as the dimension of the $L$-vector space of $K$-linear functions generated by $\left\{l_{1}, \ldots, l_{r}\right\}$. It is easy to see that $\operatorname{rank} F$ is independent of




if rank $F=\operatorname{dim}_{K} V$.

Let $K^{n}$ be the space consisting of all $n$-dimensional column vectors with entries in $K$. The vectors $\mathbf{e}_{1}=(1,0, \ldots, 0)^{T}, \ldots, \mathbf{e}_{n}=(0, \ldots, 0,1)^{T}$ form the standard basis of $K^{n}$. We shall identify a decomposable form $F$ on $K^{n}$ with the homogeneous polynomial $F(\mathbf{X})=F\left(X_{1} \mathbf{e}_{1}+\ldots+X_{n} \mathbf{e}_{n}\right) \in$ $K\left[X_{1}, \ldots, X_{n}\right]$. This homogeneous polynomial is also called a decomposable form.

Let $R$ be an integral domain (always with 1 ) with quotient field $K$. An $R$-lattice is a finitely generated $R$-submodule of a $K$-vector space. An $R$ lattice $\mathfrak{M}$ contained in the $K$-vector space $V$ is called an $R$-lattice in $V$. We define rank $\mathfrak{M}$ as the dimension $\operatorname{dim}_{K} K \mathfrak{M}$ over $K$ of the $K$-vector space $K \mathfrak{M}=\{\lambda \mathbf{x}: \lambda \in K, \mathbf{x} \in \mathfrak{M}\}$. An R-lattice decomposable form pair is a pair $(\mathfrak{M}, F)$ consisting of an $R$-lattice $\mathfrak{M}$ and a decomposable form $F$ on $K \mathfrak{M}$ of maximal rank. Two $R$-lattice decomposable form pairs $\left(\mathfrak{M}_{1}, F_{1}\right)$ and $\left(\mathfrak{M}_{2}, F_{2}\right)$ are called equivalent if there is an $R$-module isomorphism $\varphi: \mathfrak{M}_{1} \rightarrow \mathfrak{M}_{2}$ such that

$$
F_{2}(\varphi(\mathbf{x}))=F_{1}(\mathbf{x}) \quad \text { for all } \mathbf{x} \in \mathfrak{M}_{1}
$$

and weakly equivalent if there are an $R$-module isomorphism $\varphi: \mathfrak{M}_{1} \rightarrow \mathfrak{M}_{2}$ and $\left({ }^{1}\right) \lambda \in K^{*}$ such that

$$
\lambda F_{2}(\varphi(\mathbf{x}))=F_{1}(\mathbf{x}) \quad \text { for all } \mathbf{x} \in \mathfrak{M}_{1} .
$$

EXAMPLE 1 . Let $n \geq 1$ and $R^{n}$ the lattice of $n$-dimensional column vectors with entries in $R$. The group of $R$-module automorphisms of $R^{n}$ is given by $\{\mathbf{x} \mapsto U \mathbf{x}: U \in \mathrm{GL}(n, R)\}$, where $\mathrm{GL}(n, R)$ is the multiplicative group of $n \times n$ matrices with entries in $R$ and with determinant contained in the unit group $R^{*}$ of $R$. Hence two $R$-lattice decomposable form pairs $\left(R^{n}, F_{1}\right)$ and $\left(R^{n}, F_{2}\right)$ are equivalent if and only if there is a $U \in \operatorname{GL}(n, R)$ with $F_{2}(U \mathbf{x})=F_{1}(\mathbf{x})$ for $\mathbf{x} \in R^{n}$, and weakly equivalent if and only if there are $\lambda \in K^{*}$ and $U \in \mathrm{GL}(n, R)$ with $\lambda F_{2}(U \mathbf{x})=F_{1}(\mathbf{x})$ for $\mathbf{x} \in R^{n}$.

EXAmple 2. Let $M / K$ be a finite, separable extension with norm $N_{M / K}: M \rightarrow K$. Then $N_{M / K}$ is the product of the distinct $K$-isomorphisms $\alpha \mapsto \alpha^{(i)}(i=1, \ldots,[M: K])$ of $M$ which are $K$-linear functions. Hence $N_{M / K}$ is a decomposable form of maximal rank on $M$ which is called a norm form. Let $\mathfrak{M}$ be an $R$-lattice in $M$ and denote the restriction of $N_{M / K}$ to $K \mathfrak{M}$ also by $N_{M / K}$. Then $\left(\mathfrak{M}, N_{M / K}\right)$ is an $R$-lattice decomposable form pair. It is not difficult to prove that if $\mathfrak{M}_{1}, \mathfrak{M}_{2}$ are two $R$-lattices in $M$, then $\left(\mathfrak{M}_{1}, N_{M / K}\right)$ and $\left(\mathfrak{M}_{2}, N_{M / K}\right)$ are weakly equivalent if and only

$\left({ }^{1}\right)$ For any integral domain $R, R^{*}$ will denote the unit group of $R$; thus if $R$ is a field then $R^{*}=R \backslash\{0\}$. 
if there are $\mu \in M^{*}$ and a $K$-isomorphism $\sigma$ of $M$ such that

$$
\mathfrak{M}_{2}=\mu \sigma\left(\mathfrak{M}_{1}\right) \text {. }
$$

Let now $R$ be a Dedekind domain with quotient field $K$ of characteristic 0 (for instance the ring of $S$-integers of an algebraic number field). By an $R$-ideal we mean a non-zero $R$-lattice in $K ; R$-ideals contained in $R$ are said to be integral. The $R$-ideal or, more generally, $R$-lattice generated by $\alpha_{1}, \ldots, \alpha_{m}$ is denoted by $\left(\alpha_{1}, \ldots, \alpha_{m}\right)$.

By a result of Kaplansky [14] (see also [18], Ch. I, §2), every $R$-lattice of rank $n$ is isomorphic to $R^{n-1} \oplus \mathfrak{a}$ for some $R$-ideal $\mathfrak{a}$. Moreover, $R^{n-1} \oplus \mathfrak{a}$ and $R^{n-1} \oplus \mathfrak{b}$ are isomorphic if and only if $\mathfrak{a}$ and $\mathfrak{b}$ belong to the same ideal class. Let $\left\{\mathbf{e}_{1}, \ldots, \mathbf{e}_{n}\right\}$ denote, as usual, the standard basis of $K^{n}$. Since every $R$-ideal can be generated by at most two elements, every $R$-lattice $\mathfrak{M}$ of rank $n$ is isomorphic to either

$$
\begin{gathered}
\left(\mathbf{e}_{1}, \ldots, \mathbf{e}_{n}\right)=R^{n} \quad(\text { if } \mathfrak{M} \text { is free }) \quad \text { or } \\
\left(\mathbf{e}_{1}, \ldots, \mathbf{e}_{n-1}, \alpha \mathbf{e}_{n}, \beta \mathbf{e}_{n}\right) \quad(\text { if } \mathfrak{M} \text { is not free })
\end{gathered}
$$

where $\alpha, \beta \in R$ and the ideal $\mathfrak{a}=(\alpha, \beta)$ is not principal. Let $\mathfrak{M}$ be an $R$-sublattice of $R^{n}$ of rank $n$. Then for every $R$-module automorphism $\varphi$ of $\mathfrak{M}$ there is a unique $n \times n$ matrix with entries in $K$ such that $\varphi(\mathbf{x})=U \mathbf{x}$ for all $\mathbf{x} \in \mathfrak{M}$. Let $G(\mathfrak{M})$ be the group of matrices corresponding to the automorphisms of $\mathfrak{M}$. Then, trivially, two $R$-lattice decomposable form pairs $\left(\mathfrak{M}, F_{1}\right)$ and $\left(\mathfrak{M}, F_{2}\right)$ are equivalent (or weakly equivalent) if and only if there is (are) $U \in G(\mathfrak{M})$ (and $\lambda \in K^{*}$ ) such that

$$
F_{2}(\mathbf{x})=F_{1}(U \mathbf{x}) \quad\left(\lambda F_{2}(\mathbf{x})=F_{1}(U \mathbf{x}), \text { resp. }\right) \quad \text { for all } \mathbf{x} \in \mathfrak{M} .
$$

It is obvious that $G\left(R^{n}\right)=\operatorname{GL}(n, R)$. Further, the following can be easily verified: if $\mathfrak{M}=\left(\mathbf{e}_{1}, \ldots, \mathbf{e}_{n-1}, \alpha \mathbf{e}_{n}, \beta \mathbf{e}_{n}\right)$ where $\alpha, \beta \in R$ and $\mathfrak{a}=(\alpha, \beta)$ is non-principal, then

$$
\begin{aligned}
G(\mathfrak{M})= & \left\{U=\left(\begin{array}{ccc}
u_{11} & \ldots & u_{1 n} \\
\ldots \ldots & \ldots & \ldots \\
u_{n 1} & \ldots & u_{n n}
\end{array}\right):\right. \\
& u_{i j} \in R \text { for } 1 \leq i, j \leq n-1 ; u_{n n} \in R ; \\
& u_{i n} \in \mathfrak{a}^{-1} \text { for } 1 \leq i \leq n-1 ; u_{n j} \in \mathfrak{a} \text { for } 1 \leq j \leq n-1 ; \\
& \operatorname{det} U \in R^{*} .
\end{aligned}
$$

Let $(\mathfrak{M}, F)$ be an $R$-lattice decomposable form pair such that rank $\mathfrak{M}=$ $n, \operatorname{deg}(F)=r$ and $F$ has splitting field $L$, and put $V=K \mathfrak{M}$. We can factor $F$ as

$$
F(\mathbf{x})=\lambda \prod_{i=1}^{t} l_{i}(\mathbf{x})^{k_{i}} \quad \text { for all } \mathbf{x} \in V
$$


where $\lambda \in K^{*}, l_{1}, \ldots, l_{t}: V \rightarrow L$ are pairwise $L$-linearly independent linear functions and $k_{1}, \ldots, k_{t}$ are positive integers with $k_{1}+\ldots+k_{t}=r$. Let $\widehat{R}$ be the integral closure of $R$ in $L$. For any $R$-ideal $\mathfrak{b}$ in $K$ we shall write $\mathfrak{b}$ instead of $\mathfrak{b} \widehat{R}$. Let $l_{i}(\mathfrak{M})$ be the set consisting of the elements $l_{i}(\mathbf{x}), \mathbf{x} \in \mathfrak{M}$, $i=1, \ldots, t$. One can show (cf. Section 3 ) that there is an $R$-ideal $\mathfrak{c}(\mathfrak{M}, F)$, called the content of $(\mathfrak{M}, F)$, such that

$$
\mathfrak{c}(\mathfrak{M}, F)=(\lambda) \prod_{i=1}^{t}\left(l_{i}(\mathfrak{M})\right)^{k_{i}},
$$

where $(\lambda),\left(l_{i}(\mathfrak{M})\right)$ denote the $\widehat{R}$-ideals in $L$ generated by $\lambda$ and $l_{i}(\mathfrak{M})$, respectively. It is easy to check that the definition of the content is independent of the choice of $\lambda, l_{1}, \ldots, l_{t}$. It is also easy to verify that if $(\mathfrak{M}, F)$ and $\left(\mathfrak{M}^{\prime}, F^{\prime}\right)$ are equivalent $R$-lattice decomposable form pairs, then

$$
\mathfrak{c}(\mathfrak{M}, F)=\mathfrak{c}\left(\mathfrak{M}^{\prime}, F^{\prime}\right) .
$$

We now introduce the discriminant of $(\mathfrak{M}, F)$. Let $\mathcal{I}(F)$ be the collection of $L$-linearly independent subsets $\left\{l_{i_{1}}, \ldots, l_{i_{n}}\right\}$ (with $n=\operatorname{rank} \mathfrak{M}=$ $\left.\operatorname{dim}_{K} V\right)$ of $\left\{l_{1}, \ldots, l_{t}\right\}$. For $\mathcal{L}=\left\{l_{i_{1}}, \ldots, l_{i_{n}}\right\} \in \mathcal{I}(F)$, let $\mathfrak{d}(\mathfrak{M}, \mathcal{L})$ be the $\widehat{R}$-ideal in $L$ generated by all numbers

$$
\operatorname{det}\left(\begin{array}{ccc}
l_{i_{1}}\left(\mathbf{x}_{1}\right) & \cdots & l_{i_{n}}\left(\mathbf{x}_{1}\right) \\
\ldots \ldots \ldots & \ldots & \ldots \\
l_{i_{1}}\left(\mathbf{x}_{n}\right) & \cdots & l_{i_{n}}\left(\mathbf{x}_{n}\right)
\end{array}\right)
$$

with $\mathbf{x}_{1}, \ldots, \mathbf{x}_{n} \in \mathfrak{M}$. (We remark that $\mathfrak{d}(\mathfrak{M}, \mathcal{L})$ is indeed finitely generated, since there is an $\alpha \in L^{*}$ with $\left.\alpha \mathfrak{d}(\mathfrak{M}, \mathcal{L}) \subseteq \widehat{R}\right)$. The ideal

$$
\mathfrak{D}(\mathfrak{M}, F)=\prod_{\mathcal{L} \in \mathcal{I}(F)}\left(\frac{\mathfrak{d}(\mathfrak{M}, \mathcal{L})}{\left(l_{i_{1}}(\mathfrak{M})\right) \ldots\left(l_{i_{n}}(\mathfrak{M})\right)}\right)^{2}
$$

where the product is taken over all sets $\mathcal{L}=\left\{l_{i_{1}}, \ldots, l_{i_{n}}\right\}$ in $\mathcal{I}(F)$, is called the discriminant of $(\mathfrak{M}, F)$. In the trivial case $n=1$ we have $\mathfrak{D}(\mathfrak{M}, F)=(1)$. In Section 3 we shall show that $\mathfrak{D}(\mathfrak{M}, F)$ is an integral $R$-ideal. (We remark that without squaring, the ideal on the right-hand side of (1.9) would be contained in $\widehat{R}$ but not necessarily in $R$.) Further, since by assumption $F$ is of maximal rank, $\mathfrak{D}(\mathfrak{M}, F)$ is non-zero.

If the linear functions $l_{1}, \ldots, l_{t}$ are multiplied by constants $\alpha_{1}, \ldots, \alpha_{t}$, respectively, then for $\mathcal{L}=\left\{l_{i_{1}}, \ldots, l_{i_{n}}\right\} \in \mathcal{I}(F)$, both the ideals $\mathfrak{d}(\mathfrak{M}, \mathcal{L})$ and $\left(l_{i_{1}}(\mathfrak{M})\right) \ldots\left(l_{i_{n}}(\mathfrak{M})\right)$ are multiplied by $\alpha_{i_{1}}, \ldots, \alpha_{i_{n}}$. Hence the right-hand side of (1.9) does not change. This implies that the definition of $\mathfrak{D}(\mathfrak{M}, F)$ is independent of the choice of $\lambda, l_{1}, \ldots, l_{t}$. Moreover,

$$
\mathfrak{D}(\mathfrak{M}, \mu F)=\mathfrak{D}(\mathfrak{M}, F) \quad \text { for all } \mu \in K^{*} .
$$


Let now $\left(\mathfrak{M}^{\prime}, F^{\prime}\right)$ be an $R$-lattice decomposable form pair which is equivalent to $(\mathfrak{M}, F)$. Then

$$
F^{\prime}\left(\mathbf{x}^{\prime}\right)=\lambda \prod_{i=1}^{t} l_{i}^{\prime}\left(\mathbf{x}^{\prime}\right)^{k_{i}} \quad \text { for all } \mathbf{x}^{\prime} \in \mathfrak{M}^{\prime},
$$

where $l_{i}^{\prime}=l_{i} \circ \varphi^{-1}$ for $i=1, \ldots, t$ and some $R$-module isomorphism $\varphi: \mathfrak{M} \rightarrow$ $\mathfrak{M}^{\prime}$. It is trivial that $l_{i}^{\prime}\left(\mathfrak{M}^{\prime}\right)=l_{i}(\mathfrak{M})$ for $i=1, \ldots, t$ and that $\mathfrak{d}\left(\mathfrak{M}^{\prime}, \mathcal{L}^{\prime}\right)=$ $\mathfrak{d}(\mathfrak{M}, \mathcal{L})$ for all $\mathcal{L} \in \mathcal{I}(F)$, where $\mathcal{L}^{\prime}:=\left\{l_{i}^{\prime}: l_{i} \in \mathcal{L}\right\}$. Hence $\mathfrak{D}\left(\mathfrak{M}^{\prime}, F^{\prime}\right)=$ $\mathfrak{D}(\mathfrak{M}, F)$. Together with (1.10) this implies that if $(\mathfrak{M}, F),\left(\mathfrak{M}^{\prime}, F^{\prime}\right)$ are two weakly equivalent $R$-lattice decomposable form pairs, then

$$
\mathfrak{D}(\mathfrak{M}, F)=\mathfrak{D}\left(\mathfrak{M}^{\prime}, F^{\prime}\right) .
$$

EXAmple 3 . Let $F(X, Y) \in R[X, Y]$ be a binary form without multiple factors and with splitting field $L$. Then $F$ can be factored in $L[X, Y]$ as

$$
F(X, Y)=\prod_{i=1}^{r}\left(\alpha_{i} X-\beta_{i} Y\right)
$$

with $\alpha_{i}, \beta_{i} \in L$ and $\alpha_{i} \beta_{j}-\alpha_{j} \beta_{i} \neq 0$ for $1 \leq i<j \leq r$. A straightforward computation shows that the discriminant of the $R$-lattice binary form pair $\left(R^{2}, F\right)$ is equal to

$$
\mathfrak{D}\left(R^{2}, F\right)=\mathfrak{c}\left(R^{2}, F\right)^{-(2 r-2)}\left(\prod_{1 \leq i<j \leq r}\left(\alpha_{i} \beta_{j}-\alpha_{j} \beta_{i}\right)^{2}\right) .
$$

It is not difficult to prove that if $R$ is the ring of $S$-integers of an algebraic number field, then $\mathfrak{D}\left(R^{2}, F\right)$ is just the $S$-discriminant of $F$ defined in [6].

ExAmple 4. Let $F(\mathbf{X}) \in K\left[X_{1}, \ldots, X_{n}\right]$ be a decomposable form (i.e. a decomposable form on $\left.K^{n}\right)$. Then $F(\mathbf{X})=\lambda l_{1}(\mathbf{X})^{k_{1}} \ldots l_{t}(\mathbf{X})^{k_{t}}$ where $\lambda \in$ $K^{*}$ and $l_{1}, \ldots, l_{t}$ are pairwise non-proportional linear forms with coefficients in the splitting field $L$ of $F$. It is easy to verify that if $F$ is of maximal rank then

$$
\mathfrak{D}\left(R^{n}, F\right)=\prod_{\mathcal{I}(F)}\left(\frac{\operatorname{det}\left(l_{i_{1}}, \ldots, l_{i_{n}}\right)}{\left(l_{i_{1}}\right) \ldots\left(l_{i_{n}}\right)}\right)^{2},
$$

where $\mathcal{I}(F)$ is the collection of $L$-linearly independent subsets $\left\{l_{i_{1}}, \ldots, l_{i_{n}}\right\}$ of $\left\{l_{1}, \ldots, l_{t}\right\}$ and where $\left(l_{i}\right)$ denotes the $\widehat{R}$-ideal generated by the coefficients of $l_{i}$. Hence for the ring $R=\mathbb{Z}\left[\left(p_{1} \ldots p_{s}\right)^{-1}\right]$ considered in the Introduction, $\mathfrak{D}\left(R^{n}, F\right)$ is equal to $\left(D_{R}\right)$.

We now give another characterization for the discriminant. Let $(\mathfrak{M}, F)$ be an $R$-lattice decomposable form pair as above. Every $R$-ideal $\mathfrak{a}$ can be uniquely expressed as

$$
\mathfrak{a}=\prod_{\mathfrak{p}} \mathfrak{p}^{\operatorname{ord}_{\mathfrak{p}}(\mathfrak{a})},
$$


where the product is taken over all prime ideals $\mathfrak{p}$ of $R$ and where the exponents $\operatorname{ord}_{\mathfrak{p}}(\mathfrak{a})$ are integers of which at most finitely many are non-zero. For $\alpha \in K$ we put $\operatorname{ord}_{\mathfrak{p}}(\alpha)=\operatorname{ord}_{\mathfrak{p}}((\alpha))$ if $\alpha \neq 0$ and $\operatorname{ord}_{\mathfrak{p}}(\alpha)=\infty$ if $\alpha=0$. Fix a prime ideal $\mathfrak{p}$ of $R$, and let $R_{\mathfrak{p}}=\left\{\alpha \in K: \operatorname{ord}_{\mathfrak{p}}(\alpha) \geq 0\right\}$ be the local ring corresponding to $\mathfrak{p}$. Choose $\lambda \in K^{*}$ such that the decomposable form $F_{\mathfrak{M}, \mathfrak{p}}:=\lambda F$ has $\operatorname{ord}_{\mathfrak{p}}\left(\mathfrak{c}\left(\mathfrak{M}, F_{\mathfrak{M}, \mathfrak{p}}\right)\right)=0$. Note that $F_{\mathfrak{M}, \mathfrak{p}}$ maps $\mathfrak{M}$ to $R_{\mathfrak{p}}$. Denote the maximal ideal of $R_{\mathfrak{p}}$ also by $\mathfrak{p}$, and let $\overline{K_{\mathfrak{p}}}=R_{\mathfrak{p}} / \mathfrak{p}$ be the residue class field. The reduction of $\mathfrak{M} \bmod \mathfrak{p}$ is defined as the factor module

$$
\overline{\mathfrak{M}}_{\mathfrak{p}}=\mathfrak{M} / \mathfrak{p} \mathfrak{M}
$$

(where $\mathfrak{p M}=\{\lambda \mathbf{x}: \lambda \in \mathfrak{p}, \mathbf{x} \in \mathfrak{M}\})$ and the reduction of $F_{\mathfrak{M}, \mathfrak{p}} \bmod \mathfrak{p}$

$$
\bar{F}_{\mathfrak{M}, \mathfrak{p}}: \overline{\mathfrak{M}}_{\mathfrak{p}} \rightarrow \bar{K}_{\mathfrak{p}}: \mathbf{x} \bmod \mathfrak{p} \mapsto F_{\mathfrak{M}, \mathfrak{p}}(\mathbf{x}) \bmod \mathfrak{p}
$$

Note that $\overline{\mathfrak{M}}_{\mathfrak{p}}$ is a finite-dimensional $\bar{K}_{\mathfrak{p}}$-vector space and that $\bar{F}_{\mathfrak{M}, \mathfrak{p}}$ is a decomposable form on this space. The form $\bar{F}_{\mathfrak{M}, \mathfrak{p}}$ is determined by $\mathfrak{M}, F$ and $\mathfrak{p}$ up to a constant factor in $\bar{K}_{\mathfrak{p}}^{*}$.

Let $K_{0}$ be a field, $V_{0}$ a finite-dimensional $K_{0}$-vector space and $F_{0}$ : $V_{0} \rightarrow K_{0}$ a decomposable form. Further, let $\left(m_{1}, \ldots, m_{r}\right)$ be a factorization of $F_{0}$ in some extension $L_{0}$ of $K_{0}$. We denote by $N\left(F_{0}\right)$ the number of subsets $\left\{i_{1}, \ldots, i_{u}\right\}$ with $2 \leq u \leq r$ of $\{1, \ldots, r\}$ such that $\left\{m_{i_{1}}, \ldots, m_{i_{u}}\right\}$ is $L_{0}$-linearly independent. It is easy to verify that $N\left(F_{0}\right)$ is independent of the choice of the factorization $\left\{m_{1}, \ldots, m_{r}\right\}$ and that $N\left(\lambda F_{0}\right)=N\left(F_{0}\right)$ for $\lambda \in K_{0}^{*}$. In Section 3 we shall show that for every prime ideal $\mathfrak{p}$ of the Dedekind domain $R$ considered above we have

$$
\begin{gathered}
N\left(\bar{F}_{\mathfrak{M}, \mathfrak{p}}\right) \leq N(F), \\
N\left(\bar{F}_{\mathfrak{M}, \mathfrak{p}}\right)<N(F) \Leftrightarrow \operatorname{ord}_{\mathfrak{p}}(\mathfrak{D}(\mathfrak{M}, F))>0 .
\end{gathered}
$$

Let $M / K$ be a finite extension, and $\mathfrak{M}$ an $R$-lattice in $M$. We define the discriminant of $\mathfrak{M}$ by

$$
\mathfrak{D}(\mathfrak{M})=\mathfrak{D}\left(\mathfrak{M}, N_{M / K}\right) .
$$

By (1.11) and Example 2, if $\mathfrak{M}, \mathfrak{M}^{\prime}$ are $R$-lattices in $M$ such that $\mathfrak{M}^{\prime}=$ $\mu \sigma(\mathfrak{M})$ for some $\mu \in M^{*}$ and some $K$-isomorphism $\sigma$ of $M$, then

$$
\mathfrak{D}\left(\mathfrak{M}^{\prime}\right)=\mathfrak{D}(\mathfrak{M}) \text {. }
$$

ExAmple 5 . Let $\mathfrak{M}$ be a full $R$-lattice in $M$, that is, an $R$-lattice in $M$ with largest possible rank $[M: K]$. Put $n=[M: K]$ and for $\omega_{1}, \ldots, \omega_{n} \in$ $M$, denote by $D_{M / K}\left(\omega_{1}, \ldots, \omega_{n}\right)$ the discriminant of $\left\{\omega_{1}, \ldots, \omega_{n}\right\}$ (cf. [15], p. 64). Further, denote by $D_{M / K}(\mathfrak{M})$ the $R$-ideal generated by all numbers $D_{M / K}\left(\omega_{1}, \ldots, \omega_{n}\right)$ with $\omega_{1}, \ldots, \omega_{n} \in \mathfrak{M}$. Let $\widehat{R}$ be the integral closure of $R$ in $M$ and denote by $N_{M / K}(\mathfrak{M})$ the norm of the $\widehat{R}$-ideal in $M$ generated by 
$\mathfrak{M}$. It is not difficult to show that

$$
\mathfrak{D}(\mathfrak{M})=D_{M / K}(\mathfrak{M}) /\left\{N_{M / K}(\mathfrak{M})\right\}^{2} .
$$

If in particular $\mathfrak{M}$ is integral over $R$ (i.e. $\mathfrak{M} \subseteq \widehat{R}$ ), then $\mathfrak{D}(\mathfrak{M})$ divides $D_{M / K}(\mathfrak{M})$.

2. Results. Before stating our results we have to introduce heights and some notions related to $S$-integers.

The height $h(\alpha)$ of an algebraic number $\alpha$ is defined as follows: let $f(X) \in \mathbb{Z}[X]$ be an irreducible polynomial with relatively prime coefficients and with $f(\alpha)=0$, and suppose that $f(X)$ factors as $a\left(X-\alpha_{1}\right) \ldots\left(X-\alpha_{d}\right)$ over the algebraic closure of $\mathbb{Q}$ with $\alpha_{1}=\alpha$. Then

$$
h(\alpha)=\left\{|a| \prod_{i=1}^{d} \max \left(1,\left|\alpha_{i}\right|\right)\right\}^{1 / d} .
$$

The height $h(F)$ of a polynomial $F$ with algebraic coefficients is defined as the maximum of the heights of these coefficients.

Let $K$ be an algebraic number field of degree $d$. Denote by $O_{K}$ the ring of integers of $K$, and by $M_{K}$ the set of prime ideals of $O_{K}$. Take a finite set of prime ideals $S$. The ring of $S$-integers is defined by

$$
O_{S}=\left\{\alpha \in K: \operatorname{ord}_{\mathfrak{p}}(\alpha) \geq 0 \text { for all } \mathfrak{p} \in M_{K} \backslash S\right\} .
$$

The unit group of $O_{S}$ is the group of $S$-units

$$
O_{S}^{*}=\left\{\alpha \in K: \operatorname{ord}_{\mathfrak{p}}(\alpha)=0 \text { for all } \mathfrak{p} \in M_{K} \backslash S\right\} .
$$

The ring $O_{S}$ is a Dedekind domain with prime ideals $\mathfrak{p} O_{S}, \mathfrak{p} \in M_{K} \backslash S$. For convenience we shall identify the prime ideals of $O_{S}$ with those of $O_{K}$ in $M_{K} \backslash S$. We shall denote by $\left(\alpha_{1}, \ldots, \alpha_{n}\right)$ the $O_{S}$-ideal or $O_{S}$-lattice generated by $\alpha_{1}, \ldots, \alpha_{n}$, unless otherwise stated.

For every $O_{S}$-ideal $\mathfrak{a}$ there is a unique $O_{K}$-ideal $\mathfrak{a}^{*}$, composed of $O_{K}$-prime ideals outside $S$, such that $\mathfrak{a}=\mathfrak{a}^{*} O_{S}$; we put

$$
|\mathfrak{a}|_{S}=\left\{N_{K / \mathbb{Q}}\left(\mathfrak{a}^{*}\right)\right\}^{1 / d}
$$

Every $O_{S}$-ideal $\mathfrak{a}$ can be written uniquely as $\mathfrak{a}=\mathfrak{b} \cdot \mathfrak{c}^{-1}$, where $\mathfrak{b}, \mathfrak{c}$ are integral $O_{S}$-ideals with $\mathfrak{b}+\mathfrak{c}=(1)$. We put

$$
m_{S}(\mathfrak{a})=|\mathfrak{b}|_{S} \cdot|\mathfrak{c}|_{S}
$$

It is easy to show that for every $C \geq 1$ there are only finitely many $O_{S}$-ideals $\mathfrak{a}$ with $m_{S}(\mathfrak{a}) \leq C$.

Let $\mathbf{e}_{1}=(1,0, \ldots, 0)^{T}, \ldots, \mathbf{e}_{n}=(0, \ldots, 0,1)^{T}$. From the remarks made in Section 1 it follows that every $O_{S}$-lattice of rank $n$ is isomorphic to either $O_{S}^{n}=\left(\mathbf{e}_{1}, \ldots, \mathbf{e}_{n}\right)$ or $\left(\mathbf{e}_{1}, \ldots, \mathbf{e}_{n-1}, \alpha \mathbf{e}_{n}, \beta \mathbf{e}_{n}\right)$ where $\mathfrak{a}=(\alpha, \beta)$ is an integral, non-principal $O_{S}$-ideal. Here $\mathfrak{a}$ can be replaced by any ideal belonging to 
the same $O_{S}$-ideal class as a. By Lemma 5 in Section 4 of this paper, every non-principal $O_{S^{-}}$-ideal class contains an integral $O_{S^{-}}$ideal $(\alpha, \beta)$ such that

$$
h(\alpha) \leq C_{1}, \quad h(\beta) \leq C_{1},
$$

where $C_{1}$ is an effectively computable number depending only on $d=[K: \mathbb{Q}]$ and the discriminant $D_{K}$ of $K$. We conclude that every $O_{S}$-lattice of rank $n$ is isomorphic to either

$$
\begin{cases}\left(\mathbf{e}_{1}, \ldots, \mathbf{e}_{n}\right)=O_{S}^{n} \quad \text { or } & \\ \left(\mathbf{e}_{1}, \ldots, \mathbf{e}_{n-1}, \alpha \mathbf{e}_{n}, \beta \mathbf{e}_{n}\right) & \text { with } \alpha, \beta \in O_{S}, h(\alpha) \leq C_{1} \\ & h(\beta) \leq C_{1},(\alpha, \beta) \text { non-principal. }\end{cases}
$$

The lattices in $(2.3)$ are called reduced. If $(\mathfrak{M}, F)$ is an $O_{S}$-lattice decomposable form pair in which $\mathfrak{M}$ is reduced and rank $\mathfrak{M}=n$, then $F$ is a decomposable form on $K^{n}$. The height of $F$ is defined as the height of the corresponding polynomial $F(\mathbf{X})=F\left(X_{1} \mathbf{e}_{1}+\ldots+X_{n} \mathbf{e}_{n}\right) \in K\left[X_{1}, \ldots, X_{n}\right]$.

We are now in a position to state our results. By $D_{M}$ we denote the discriminant of a number field $M$. As before, we put $d=[K: \mathbb{Q}]$. Further, let $s$ denote the cardinality of $S$, and $P$ the largest of the prime numbers lying below the prime ideals in $S$ with $P=1$ if $S=\emptyset$. Finally, let $L$ be a finite, normal extension of $K$, let $r$ and $n$ be positive integers, and let $\mathfrak{d}$ be a non-zero integral $O_{S}$-ideal.

Theorem 2. Let $(\mathfrak{M}, F)$ be an $O_{S}$-lattice decomposable form pair such that rank $\mathfrak{M}=n, \operatorname{deg}(F)=r, F$ has splitting field $L$ and $\mathfrak{D}(\mathfrak{M}, F)=\mathfrak{d}$. Then $(\mathfrak{M}, F)$ is weakly equivalent to a pair $\left(\mathfrak{M}^{\prime}, F^{\prime}\right)$, where $\mathfrak{M}^{\prime}$ is a reduced $O_{S}$-lattice of rank $n$, and $F^{\prime}$ is a decomposable form on $K^{n}$ with

$$
h\left(F^{\prime}\right) \leq C_{2}|\mathfrak{d}|_{S}^{C_{3}},
$$

where $C_{2}, C_{3}$ are effectively computable numbers depending only on $d,\left|D_{L}\right|$, $s, P, n$ and $r$.

In [6], we proved Theorem 2 in the case that $\mathfrak{M}=O_{S}^{2}$ and $F$ is a binary form, and gave explicit expressions for $C_{2}$ and $C_{3}$.

The main tool in the proof of Theorem 2 is an effective result of Györy ([10], Lemma 6) on the $S$-unit equation $\alpha x+\beta y=1$ in $x, y \in O_{S}^{*}$. This result of Györy was proved by means of Baker's theory on linear forms in logarithms of algebraic numbers and its $p$-adic analogue.

We now state some consequences of Theorem 2 which will be proved in Section 7. The upper bound for $h\left(F^{\prime}\right)$ in Theorem 2 depends on $\left|D_{L}\right|$. In Section 7 we shall prove that $\left|D_{L}\right| \leq C_{4}$, where $C_{4}$ is an effectively computable number depending only on $d,\left|D_{K}\right|, s, P, n, r$ and $|\mathfrak{d}|_{S}$. Thus we obtain the following. 
Corollary 1. Let $(\mathfrak{M}, F)$ be as in Theorem 2. Then $(\mathfrak{M}, F)$ is weakly equivalent to a pair $\left(\mathfrak{M}^{\prime}, F^{\prime}\right)$, where $\mathfrak{M}^{\prime}$ is a reduced $O_{S}$-lattice of rank $n$, and $F^{\prime}$ is a decomposable form on $K^{n}$ with

$$
h\left(F^{\prime}\right) \leq C_{5},
$$

where $C_{5}$ is an effectively computable number depending only on $d,\left|D_{K}\right|, s$, $P, n, r$ and $|\mathfrak{d}|_{S}$.

We note that for $n=2$ and $\mathfrak{M}=\mathfrak{M}^{\prime}=O_{S}^{2}$, our Corollary 1 (see also Example 3 in Section 1) implies, in a less explicit form, Theorem 2 of [6].

Assume that $K$ is effectively given, i.e. that an irreducible polynomial $f(X) \in \mathbb{Z}[X]$ is given such that $K \cong \mathbb{Q}[X] /(f(X))$. Let $\alpha$ be a zero of $f$. Then every element of $K$ can be expressed uniquely as $\left(\sum_{i=0}^{d-1} a_{i} \alpha^{i}\right) / a_{d}(d=$ $\operatorname{deg}(f))$, where $a_{0}, \ldots, a_{d-1}, a_{d}$ are rational integers with $\operatorname{gcd}\left(a_{0}, \ldots, a_{d-1}\right.$, $\left.a_{d}\right)=1$ and $a_{d}>0$; the tuple $\left(a_{0}, \ldots, a_{d}\right)$ is called a representation of the element in question. We say that an element of $K$ is given (or computable) if the finite tuple of integers by which it is represented is given (or can be computed). Then sums, differences, products and quotients of given elements in $K$ can be computed. We assume that $S$ is effectively given in the sense that for every prime ideal in $S$, a set of generators is given. Then for every given $\alpha \in K$ it can be effectively decided whether $\alpha \in O_{S}$ (or $\left.\alpha \in O_{S}^{*}\right)$.

Corollary 2. For any positive integers $n$ and $r$ and every integral $O_{S^{-}}$ ideal $\mathfrak{d}$, there are only finitely many weak equivalence classes of $O_{S}$-lattice decomposable form pairs $(\mathfrak{M}, F)$ such that rank $\mathfrak{M}=n, \operatorname{deg}(F)=r$ and $\mathfrak{D}(\mathfrak{M}, F)=\mathfrak{d}$. Further, if a set of generators for $\mathfrak{d}$ is given, then a full set of representatives of these weak equivalence classes can be effectively determined.

Corollary 2 does not follow at once from Corollary 1 , since if $\left(\mathfrak{M}_{1}, F_{1}\right)$ and $\left(\mathfrak{M}_{2}, F_{2}\right)$ are two $O_{S}$-lattice decomposable form pairs such that $\mathfrak{M}_{1}$ and $\mathfrak{M}_{2}$ are reduced and $F_{1}$ and $F_{2}$ have small heights, then it might still happen that $\left(\mathfrak{M}_{1}, F_{1}\right)$ and $\left(\mathfrak{M}_{2}, F_{2}\right)$ are weakly equivalent. We shall prove that there is an algorithm to decide whether two such pairs $\left(\mathfrak{M}_{1}, F_{1}\right),\left(\mathfrak{M}_{2}, F_{2}\right)$ are weakly equivalent or not.

By combining Corollary 1 with (1.12) we get

Corollary 3. Let $(\mathfrak{M}, F)$ be an $O_{S}$-lattice decomposable form pair such that rank $\mathfrak{M}=n, \operatorname{deg}(F)=r$ and $N\left(\bar{F}_{\mathfrak{M}, \mathfrak{p}}\right)=N(F)$ for every prime ideal $\mathfrak{p}$ of $O_{S}$. Then $(\mathfrak{M}, F)$ is weakly equivalent to a pair $\left(\mathfrak{M}^{\prime}, F^{\prime}\right)$ such that $\mathfrak{M}^{\prime}$ is reduced and $h\left(F^{\prime}\right) \leq C_{6}$, where $C_{6}$ is an effectively computable number depending only on $d,\left|D_{K}\right|, s, P, n$, and $r$. 
We now state some results on (not weak) equivalence classes of $O_{S}$-lattice decomposable form pairs. Let $\mathfrak{c}$ be a non-zero $O_{S}$-ideal.

Corollary 4. Let $(\mathfrak{M}, F)$ be an $O_{S}$-lattice decomposable form pair such that rank $\mathfrak{M}=n, \operatorname{deg}(F)=r, F$ has splitting field $L, \mathfrak{D}(\mathfrak{M}, F)=\mathfrak{d}$ and $\mathfrak{c}(\mathfrak{M}, F)=\mathfrak{c}$. Then $(\mathfrak{M}, F)$ is equivalent to a pair $\left(\mathfrak{M}^{\prime}, F^{\prime}\right)$, where $\mathfrak{M}^{\prime}$ is a reduced $O_{S}$-lattice of rank $n$, and $F^{\prime}$ is a decomposable form on $K^{n}$ with

$$
h\left(F^{\prime}\right) \leq C_{7} m_{S}(\mathfrak{c})|\mathfrak{d}|_{S}^{C_{8}} \quad \text { and } \quad h\left(F^{\prime}\right) \leq C_{9} m_{S}(\mathfrak{c}),
$$

where $C_{7}, C_{8}, C_{9}$ are effectively computable numbers such that $C_{7}, C_{8}$ depend only on $d,\left|D_{L}\right|, s, P, n$ and $r$, and $C_{9}$ only on $d,\left|D_{K}\right|, s, P, n, r$ and $|\mathfrak{d}|_{S}$.

Corollary 4 implies that there are only finitely many equivalence classes of $O_{S}$-lattice decomposable form pairs $(\mathfrak{M}, F)$ with $\operatorname{rank} \mathfrak{M}=n, \operatorname{deg}(F)=$ $r, \mathfrak{c}(\mathfrak{M}, F)=\mathfrak{c}$ and $\mathfrak{D}(\mathfrak{M}, F)=\mathfrak{d}$. Further, by arguments similar to the proof of Corollary 2 one can prove the existence of an effective algorithm that selects one pair $(\mathfrak{M}, F)$ from each of these equivalence classes. We remark that in view of Example 4 of Section 1, Theorem 1 stated in the Introduction is exactly Corollary 4 with the second inequality for $K=\mathbb{Q}$, $O_{S}=R=\mathbb{Z}\left[\left(p_{1} \ldots p_{s}\right)^{-1}\right], \mathfrak{M}=\mathfrak{M}^{\prime}=R^{n}, \mathfrak{c}=(1)$ and $\mathfrak{d}=\left(D_{R}(F)\right)=(D)$.

From Corollary 4 we shall derive the following.

Corollary 5. Let $(\mathfrak{M}, F)$ be as in Corollary 4. Then $\mathfrak{M}=\left(\omega_{1}, \ldots, \omega_{m}\right)$ where either $\mathfrak{M}$ is free and $m=n$, or $\mathfrak{M}$ is not free, $m=n+1$ and $\omega_{n+1}=\gamma \omega_{n}$ for some $\gamma \in K^{*}$ with $h(\gamma) \leq C_{10}, F\left(\omega_{1}\right) F\left(\omega_{2}\right) \ldots F\left(\omega_{m}\right) \neq 0$ and

$$
h\left(F\left(\omega_{i}\right)\right) \leq C_{11} m_{S}(\mathfrak{c})|\mathfrak{d}|_{S}^{C_{12}} \quad \text { and } \quad h\left(F\left(\omega_{i}\right)\right) \leq C_{13} m_{S}(\mathfrak{c})
$$

for $i=1, \ldots, m$, where $C_{10}, C_{11}, C_{12}, C_{13}$ are effectively computable numbers such that $C_{10}$ depends only on $d$ and $\left|D_{K}\right|, C_{11}$ and $C_{12}$ only on $d$, $\left|D_{L}\right|, s, P, n$ and $r$, and $C_{13}$ only on $d,\left|D_{K}\right|, s, P, n, r$ and $|\mathfrak{d}|_{S}$.

A trivial consequence of Corollary 5 is that the bounds occurring on the right-hand side of the estimates in (2.4) are upper bounds for

$$
\min \{h(F(\mathbf{x})): \mathbf{x} \in \mathfrak{M}, F(\mathbf{x}) \neq 0\} .
$$

Hence, for $n=2$ and $\mathfrak{M}=O_{S}^{2}$, Corollary 5 implies (in a less explicit form) Corollary 5 of $[6]$.

Let $M / K$ be a finite extension of degree $r$. Two $O_{S^{-}}$-lattices $\mathfrak{M}_{1}, \mathfrak{M}_{2}$ in $M$ are said to be similar if $\mathfrak{M}_{2}=\mu \mathfrak{M}_{1}$ for some $\mu \in M^{*}$.

Corollary 6. Let $\mathfrak{M}$ be an $O_{S}$-lattice of rank $n$ in $M$ with $\mathfrak{D}(\mathfrak{M})=\mathfrak{d}$. Then $\mathfrak{M}$ is similar to an $O_{S}$-lattice $\left(\omega_{1}, \ldots, \omega_{m}\right)$ with $\omega_{1}, \ldots, \omega_{m} \in M^{*}$, where either $\mathfrak{M}$ is free and $m=n$, or $\mathfrak{M}$ is not free, $m=n+1$ and $\omega_{n+1}=\gamma \omega_{n}$ for some $\gamma \in K^{*}$ with $h(\gamma) \leq C_{14}$ and

$$
h\left(\omega_{i}\right) \leq C_{15}|\mathfrak{d}|_{S}^{C_{16}} \quad \text { and } \quad h\left(\omega_{i}\right) \leq C_{17} \quad \text { for } i=1, \ldots, m,
$$


where $C_{14}, C_{15}, C_{16}, C_{17}$ are effectively computable numbers such that $C_{14}$ depends only on $d$ and $\left|D_{K}\right|, C_{15}, C_{16}$ only on $d,\left|D_{M}\right|, s, P, n$ and $r=$ $[M: K]$, and $C_{17}$ only on $d,\left|D_{K}\right|, s, P, n, r$ and $|\mathfrak{d}|_{S}$.

We say that an $O_{S}$-lattice $\mathfrak{M}$ is of degree $r$ over $K$ if it is contained in some finite extension of $K$, and the smallest extension of $K$ containing $\mu \mathfrak{M}$ for some non-zero algebraic number $\mu$ has degree $r$ over $K$. Corollary 6 implies that there are only finitely many similarity classes of $O_{S}$-lattices $\mathfrak{M}$ of degree $r$ and rank $n$ with $\mathfrak{D}(\mathfrak{M})=\mathfrak{d}$. Further, in view of (1.5) it is easy to prove the existence of an algorithm to choose such $\mathfrak{M}$ from each of these similarity classes.

We note that from Corollary 6 and relation (1.13) one can also deduce effective finiteness results for full, integral $O_{S}$-lattices $\mathfrak{M}$ of given (finite) rank and given (non-zero) ordinary discriminant $D(\mathfrak{M})$. We shall not work these out here. For $K=\mathbb{Q}$ and $S=\emptyset$, these imply a result of Nagell ([17], Theorem 6) which says that there are only finitely many full and integral $\mathbb{Z}$-modules with a given (finite) rank and a given (non-zero) ordinary discriminant, and all these $\mathbb{Z}$-modules can be effectively determined.

3. Properties of decomposable forms and discriminants. In this section, we prove the facts about decomposable forms and discriminants mentioned in Section 1; namely, that $\mathfrak{c}(\mathfrak{M}, F)$ is an $R$-ideal, that $\mathfrak{D}(\mathfrak{M}, F)$ is an integral $R$-ideal, and that $\mathfrak{D}(\mathfrak{M}, F)$ satisfies (1.12), and some other facts needed in this paper.

Let $R$ be a Dedekind domain with quotient field $K$ of characteristic 0 , and $(\mathfrak{M}, F)$ an $R$-lattice decomposable form pair such that rank $\mathfrak{M}=n$, $\operatorname{deg}(F)=r$ and $F$ has splitting field $L$. Let $\operatorname{Gal}(L / K)$ denote the Galois group of $L / K$. Since $F$ maps $K \mathfrak{M}$ to $K$, it can be factored as

$$
F(\mathbf{x})=\lambda \prod_{i=1}^{t} l_{i}(\mathbf{x})^{k_{i}} \quad \text { for } \mathbf{x} \in K \mathfrak{M},
$$

where $\lambda \in K^{*}, l_{1}, \ldots, l_{t}: V \rightarrow L$ are pairwise non-proportional linear functions and $k_{1}, \ldots, k_{t}$ are positive integers such that

$$
\sigma \circ l_{i}=l_{\sigma(i)}, \quad k_{\sigma(i)}=k_{i} \quad \text { for } i=1, \ldots, t, \sigma \in \operatorname{Gal}(L / K),
$$

where $(\sigma(1), \ldots, \sigma(t))$ is a permutation of $(1, \ldots, t)$ for $\sigma \in \operatorname{Gal}(L / K)$. Define the fields $M_{i}(i=1, \ldots, t)$ by

$$
\operatorname{Gal}\left(L / M_{i}\right)=\{\sigma \in \operatorname{Gal}(L / K): \sigma(i)=i\} .
$$

Then $l_{i}(\mathfrak{M}) \subset M_{i}$ for $i=1, \ldots, t$. Partition $\{1, \ldots, t\}$ into $\operatorname{Gal}(L / K)$ orbits $\mathcal{C}_{1}, \ldots, \mathcal{C}_{u}$ such that $i$ and $j$ belong to the same orbit if and only if $\sigma(i)=j$ for some $\sigma \in \operatorname{Gal}(L / K)$. For convenience, we assume that $i \in \mathcal{C}_{i}$ for $i=1, \ldots, u$. We shall frequently use the following fact: 
let $S_{1}, \ldots, S_{t}$ be non-empty sets such that $S_{i} \subseteq M_{i}$ and $\sigma\left(S_{i}\right)=$ $S_{\sigma(i)}$ for $i=1, \ldots, t, \sigma \in \operatorname{Gal}(L / K)$; then it is possible to choose $\pi_{i}$ from $S_{i}$ such that $\sigma\left(\pi_{i}\right)=\pi_{\sigma(i)}$ for $i=1, \ldots, t, \sigma \in \operatorname{Gal}(L / K)$.

Namely, for $i=1, \ldots, u$ one can choose $\pi_{i}$ arbitrarily from $S_{i}$ and then the remaining $\pi_{i}$ can be selected such that the relationships $\sigma\left(\pi_{i}\right)=\pi_{\sigma(i)}$ for $i=1, \ldots, u, \sigma \in \operatorname{Gal}(L / K)$ are all satisfied. This is possible since $S_{i} \subseteq M_{i}$ for $i=1, \ldots, t$.

Let $\mathfrak{p}$ be a prime ideal of $R$. As before, we put

$$
R_{\mathfrak{p}}=\left\{\alpha \in K: \operatorname{ord}_{\mathfrak{p}}(\alpha) \geq 0\right\}, \quad \mathfrak{M}_{\mathfrak{p}}=R_{\mathfrak{p}} \mathfrak{M},
$$

and denote the maximal ideal of $R_{\mathfrak{p}}$ also by $\mathfrak{p}$. Let $\widehat{R}, \widehat{R}_{\mathfrak{p}}$ denote the integral closures of $R, R_{\mathfrak{p}}$, respectively, in $L$, and let $\mathfrak{P}_{1}, \ldots, \mathfrak{P}_{g}$ be the prime ideals of $\widehat{R}$ lying above $\mathfrak{p}$. In what follows, we denote the $\widehat{R}_{\mathfrak{p}}$-ideal generated by $\alpha_{1}, \ldots, \alpha_{r}$ by $\left(\alpha_{1}, \ldots, \alpha_{r}\right)_{\mathfrak{p}}$. Further, the $\widehat{R}_{\mathfrak{p}}$-ideal generated by the numbers $l_{i}(\mathbf{x}), \mathbf{x} \in \mathfrak{M}$, is denoted by $\left(l_{i}(\mathfrak{M})\right)_{\mathfrak{p}}$. Note that $l_{i}(\mathbf{x}) \in M_{i}$ for $\mathbf{x} \in \mathfrak{M}$. Both $\widehat{R}_{\mathfrak{p}}$ and $\widehat{R}_{i, \mathfrak{p}}:=\widehat{R}_{\mathfrak{p}} \cap M_{i}$ are principal ideal domains (cf. [2], Ch. III, $\S 4$ ). Hence $\left(l_{i}(\mathfrak{M})\right)_{\mathfrak{p}}$ is generated by an element in $M_{i}$. In other words, there are $\pi_{i} \in M_{i}$ such that

$$
\left(l_{i}(\mathfrak{M})\right)_{\mathfrak{p}}=\left(\pi_{i}\right)_{\mathfrak{p}} \quad \text { for } i=1, \ldots, t .
$$

By applying (3.4) to the sets $\left\{\xi_{i} \in M_{i}:\left(l_{i}(\mathfrak{M})\right)_{\mathfrak{p}}=\left(\xi_{i}\right)_{\mathfrak{p}}\right\}$ we infer that $\pi_{1}, \ldots, \pi_{t}$ can be chosen such that

$$
\sigma\left(\pi_{i}\right)=\pi_{\sigma(i)} \quad \text { for } i=1, \ldots, t, \sigma \in \operatorname{Gal}(L / K) .
$$

Put $\pi=\pi_{1}^{k_{1}} \ldots \pi_{t}^{k_{t}}$. Then $\sigma(\pi)=\pi$ for each $\sigma \in \operatorname{Gal}(L / K)$, hence $\pi \in K$. Further,

$$
\operatorname{ord}_{\mathfrak{P}_{j}}(\mathfrak{c}(\mathfrak{M}, F))=\operatorname{ord}_{\mathfrak{P}_{j}}(\pi) \quad \text { for } j=1, \ldots, g .
$$

Hence $\mathfrak{c}(\mathfrak{M}, F)$ is an $R$-ideal.

Define the linear functions $m_{i}=\pi_{i}^{-1} l_{i}: \mathfrak{M} \rightarrow L(i=1, \ldots, t)$. Then, by (3.2), (3.5) and (3.6),

$$
\begin{aligned}
\sigma \circ m_{i}=m_{\sigma(i)}, \quad\left(m_{i}(\mathfrak{M})\right)_{\mathfrak{p}}= & (1)_{\mathfrak{p}} \\
& \text { for } i=1, \ldots, t, \sigma \in \operatorname{Gal}(L / K) .
\end{aligned}
$$

Since $R_{\mathfrak{p}}$ is a principal ideal domain, $\mathfrak{M}_{\mathfrak{p}}$ is a free $R_{\mathfrak{p}}$-module of rank $n$ (cf. [18], Ch. I, §2). Hence $\mathfrak{M}_{\mathfrak{p}}$ has an $R_{\mathfrak{p}}$-basis $\left\{\mathbf{x}_{1}, \ldots, \mathbf{x}_{n}\right\}$ with $\mathbf{x}_{i} \in \mathfrak{M}$ for $i=1, \ldots, n$. Let $\mathcal{I}^{\prime}(F)$ be the collection of $L$-linearly independent subsets $\left\{m_{i_{1}}, \ldots, m_{i_{n}}\right\}(n=\operatorname{rank} \mathfrak{M})$ of $\left\{m_{1}, \ldots, m_{t}\right\}$. Define the number

$$
\delta_{\mathfrak{p}}=\prod_{\mathcal{I}^{\prime}(F)}\left\{\operatorname{det}\left(m_{i_{k}}\left(\mathbf{x}_{j}\right)\right)_{1 \leq k, j \leq n}\right\}^{2},
$$


where the product is taken over all sets $\left\{m_{i_{1}}, \ldots, m_{i_{n}}\right\}$ in $\mathcal{I}^{\prime}(F)$. From (1.10), (3.7) and the fact that $\left\{\mathbf{x}_{1}, \ldots, \mathbf{x}_{n}\right\}$ is an $R_{\mathfrak{p}}$-basis of $\mathfrak{M}_{\mathfrak{p}}$, it follows that

$$
\operatorname{ord}_{\mathfrak{P}_{i}}\left(\delta_{\mathfrak{p}}\right)=\operatorname{ord}_{\mathfrak{P}_{i}}(\mathfrak{D}(\mathfrak{M}, F)) \quad \text { for } i=1, \ldots, g .
$$

We notice that $\delta_{\mathfrak{p}} \in \widehat{R}_{\mathfrak{p}}$, since $\left(m_{i}(\mathfrak{M})\right)_{\mathfrak{p}}=(1)_{\mathfrak{p}}$ for $i=1, \ldots, t$. Further, $\left\{\sigma \circ m_{i_{1}}, \ldots, \sigma \circ m_{i_{n}}\right\}$ is $L$-linearly independent if and only if $\left\{m_{i_{1}}, \ldots, m_{i_{n}}\right\}$ is. Hence each $\sigma \in \operatorname{Gal}(L / K)$ permutes the sets of $\mathcal{I}^{\prime}(F)$. Moreover, each factor on the right-hand side of (3.8) depends only on the set $\left\{m_{i_{1}}, \ldots, m_{i_{n}}\right\}$ and not on its ordering because of the exponent 2 . It follows that each $\sigma \in \operatorname{Gal}(L / K)$ permutes the factors on the right-hand side of (3.8), which implies that $\sigma\left(\delta_{\mathfrak{p}}\right)=\delta_{\mathfrak{p}}$. Therefore, $\delta_{\mathfrak{p}} \in \widehat{R}_{\mathfrak{p}} \cap K=R_{\mathfrak{p}}$. We conclude that $\mathfrak{D}(\mathfrak{M}, F)$ is an integral $R$-ideal.

We now prove formula (1.12). We recall that $\overline{\mathfrak{M}}_{\mathfrak{p}}=\mathfrak{M} / \mathfrak{p} \mathfrak{M}$. We take

$$
F_{\mathfrak{M}, \mathfrak{p}}(\mathbf{x})=\prod_{i=1}^{t} m_{i}(\mathbf{x})^{k_{i}} \quad \text { for } \mathbf{x} \in K \mathfrak{M},
$$

which can be done since $\left(m_{i}(\mathfrak{M})\right)_{\mathfrak{p}}=(1)_{\mathfrak{p}}$ for $i=1, \ldots, t$. By $\bar{F}_{\mathfrak{M}, \mathfrak{p}}$ we denote the decomposable form on $\overline{\mathfrak{M}}_{\mathfrak{p}}: \mathbf{x} \bmod \mathfrak{p} \mapsto F_{\mathfrak{M}, \mathfrak{p}}(\mathbf{x}) \bmod \mathfrak{p}$.

Lemma 1. We have $N\left(\bar{F}_{\mathfrak{M}, \mathfrak{p}}\right) \leq N(F)$. Further, $N\left(\bar{F}_{\mathfrak{M}, \mathfrak{p}}\right)<N(F)$ if and only if $\operatorname{ord}_{\mathfrak{p}}(\mathfrak{D}(\mathfrak{M}, F))>0$.

Proof. Let $\mathfrak{P}$ be one of the prime ideals of $\widehat{R}$ lying above $\mathfrak{p}$. Put $\bar{K}_{\mathfrak{p}}=R / \mathfrak{p} \cong R_{\mathfrak{p}} / \mathfrak{p}$ and $\bar{L}_{\mathfrak{P}}=\widehat{R} / \mathfrak{P} \cong \widehat{R}_{\mathfrak{p}} / \mathfrak{P}$. Since, by $(3.7), m_{i}(\mathfrak{M}) \subseteq \widehat{R}_{\mathfrak{p}}$ for $i=1, \ldots, t$, we can define the reductions of $m_{i} \bmod \mathfrak{P}$ by

$$
\bar{m}_{i}(\mathbf{x} \bmod \mathfrak{p} \mathfrak{M})=m_{i}(\mathbf{x}) \bmod \mathfrak{P} .
$$

Then

$$
\bar{F}_{\mathfrak{M}, \mathfrak{p}}(\mathbf{x})=\prod_{i=1}^{t} \bar{m}_{i}(\mathbf{x})^{k_{i}} \quad \text { for } \mathbf{x} \in \overline{\mathfrak{M}}_{\mathfrak{p}} .
$$

Obviously, if $\left\{\bar{m}_{i_{1}}, \ldots, \bar{m}_{i_{u}}\right\}$ is some $\bar{L}_{\mathfrak{P}^{-}}$-linearly independent subset of $\left\{\bar{m}_{1}, \ldots, \bar{m}_{t}\right\}$ then $\left\{m_{i_{1}}, \ldots, m_{i_{u}}\right\}$ is $L$-linearly independent. Hence $N\left(\bar{F}_{\mathfrak{M}, \mathfrak{p}}\right) \leq N(F)$. Further, $N\left(\bar{F}_{\mathfrak{M}, \mathfrak{p}}\right)<N(F)$ if and only if there is an $L$-linearly independent subset $\left\{m_{i_{1}}, \ldots, m_{i_{u}}\right\}$ of $\left\{m_{1}, \ldots, m_{t}\right\}$ with $u \geq 2$ such that $\left\{\bar{m}_{i_{1}}, \ldots, \bar{m}_{i_{u}}\right\}$ is $\bar{L}_{\mathfrak{P} \text {-linearly dependent. But each linearly inde- }}$ pendent subset of $\left\{m_{1}, \ldots, m_{t}\right\}$ can be extended to a linearly independent subset of cardinality $n$. Hence $N\left(\bar{F}_{\mathfrak{M}, \mathfrak{p}}\right)<N(F)$ if and only if there is a set $\left\{m_{i_{1}}, \ldots, m_{i_{n}}\right\}$ in $\mathcal{I}^{\prime}(F)$ with $u \geq 2$ such that $\left\{\bar{m}_{i_{1}}, \ldots, \bar{m}_{i_{n}}\right\}$ is $\bar{L}_{\mathfrak{P}}$-linearly dependent. But $\left\{\bar{m}_{i_{1}}, \ldots, \bar{m}_{i_{n}}\right\}$ is $\bar{L}_{\mathfrak{P}}$-linearly dependent if and only if

$$
\operatorname{ord}_{\mathfrak{P}}\left(\operatorname{det}\left(m_{i_{k}}\left(\mathbf{x}_{j}\right)\right)_{i \leq k, j \leq n}\right)>0 \text {, }
$$


where $\left\{\mathbf{x}_{1}, \ldots, \mathbf{x}_{n}\right\}$ is the $R_{\mathfrak{p}}$-basis of $\mathfrak{M}_{\mathfrak{p}}$ used in the definition of $\delta_{\mathfrak{p}}$. This shows that $N\left(\bar{F}_{\mathfrak{M}, \mathfrak{p}}\right)<N(F)$ if and only if $\operatorname{ord}_{\mathfrak{P}}\left(\delta_{\mathfrak{p}}\right)>0$. Together with (3.9) this implies Lemma 1.

As before, $\mathcal{C}_{1}, \ldots, \mathcal{C}_{u}$ denote the $\operatorname{Gal}(L / K)$-orbits of $\{1, \ldots, r\}$ where the action of $\operatorname{Gal}(L / K)$ on $\{1, \ldots, u\}$ is defined by (3.2). Further, $i \in \mathcal{C}_{i}$ for $i=1, \ldots, t$. Let $\widehat{R}_{i}$ be the integral closure of $R$ in the field $M_{i}$ defined by (3.3) and let $\mathfrak{d}_{i}$ be the discriminant of the ring extension $\widehat{R}_{i} / R$ (see e.g. [13]).

Lemma 2. We have $\mathfrak{D}(\mathfrak{M}, F)^{n} \subseteq\left(\mathfrak{d}_{1} \ldots \mathfrak{d}_{u}\right)^{2}$.

Proof. It suffices to prove that for every prime ideal $\mathfrak{p}$ of $R$,

$$
n \operatorname{ord}_{\mathfrak{p}}\left(\delta_{\mathfrak{p}}\right) \geq 2 \sum_{i=1}^{u} \operatorname{ord}_{\mathfrak{p}}\left(\mathfrak{d}_{i}\right),
$$

where $\delta_{\mathfrak{p}}$ is defined by (3.8) for some $R_{\mathfrak{p}}$-basis $\left\{\mathbf{x}_{1}, \ldots, \mathbf{x}_{n}\right\}$ of $\mathfrak{M}_{\mathfrak{p}}$ with $\mathbf{x}_{i} \in \mathfrak{M}$ for $i=1, \ldots, n$. Fix a prime ideal $\mathfrak{p}$, and consider one of the factors

$$
\Delta=\operatorname{det}\left(\left(m_{i_{k}}\left(\mathbf{x}_{j}\right)\right)_{1 \leq k, j \leq n}\right)
$$

in (3.8) where $\left\{m_{i_{1}}, \ldots, m_{i_{n}}\right\} \in \mathcal{I}^{\prime}(F)$. Let $\mathbf{m}_{i}$ be the vector with coordinates $\left(m_{i}\left(\mathbf{x}_{1}\right), \ldots, m_{i}\left(\mathbf{x}_{n}\right)\right)$. In what follows, if $\mathbf{a}$ is any vector with coordinates in $L$, then $(\mathbf{a})_{\mathfrak{p}}$ denotes the $\widehat{R}_{\mathfrak{p}}$-ideal generated by the coordinates of a. Thus, using (3.7), we get

$$
\begin{aligned}
(\Delta)_{\mathfrak{p}} & =\left(\operatorname{det}\left(\mathbf{m}_{i_{1}}, \ldots, \mathbf{m}_{i_{n}}\right)\right)_{\mathfrak{p}}=\left(\operatorname{det}\left(\mathbf{m}_{i_{1}}, \mathbf{m}_{i_{2}}-\mathbf{m}_{i_{1}}, \ldots, \mathbf{m}_{i_{n}}-\mathbf{m}_{i_{1}}\right)\right)_{\mathfrak{p}} \\
& \subseteq\left(\mathbf{m}_{i_{2}}-\mathbf{m}_{i_{1}}\right)_{\mathfrak{p}} \ldots\left(\mathbf{m}_{i_{n}}-\mathbf{m}_{i_{1}}\right)_{\mathfrak{p}} .
\end{aligned}
$$

We can do the same for $\mathbf{m}_{i_{2}}, \ldots, \mathbf{m}_{i_{n}}$ in the rôle of $\mathbf{m}_{i_{1}}$. Thus

$$
(\Delta)_{\mathfrak{p}}^{n} \subseteq \prod_{\substack{1 \leq k, l \leq n \\ k \neq l}}\left(\mathbf{m}_{i_{k}}-\mathbf{m}_{i_{l}}\right)_{\mathfrak{p}} .
$$

It is easy to see that every pair $\left\{\mathbf{m}_{i}, \mathbf{m}_{j}\right\}$ with distinct $i, j \in\{1, \ldots, t\}$ is contained in one of the sets of $\mathcal{I}^{\prime}(F)$. Since, by (3.7), each vector $\mathbf{m}_{i}$ has its coordinates in $\widehat{R}_{\mathfrak{p}}$, it follows that

$$
(\delta)_{\mathfrak{p}}^{n} \subseteq\left\{\prod_{\substack{1 \leq i, j \leq n \\ i \neq j}}\left(\mathbf{m}_{i}-\mathbf{m}_{j}\right)_{\mathfrak{p}}\right\}^{2} .
$$

This implies that

$$
\left(\delta_{\mathfrak{p}}\right)_{\mathfrak{p}}^{n} \subseteq \prod_{k=1}^{u}\left\{\prod_{\substack{i, j \in \mathcal{C}_{k} \\ i \neq j}}\left(\mathbf{m}_{i}-\mathbf{m}_{j}\right)_{\mathfrak{p}}\right\}^{2}
$$


where the second product takes the value 1 if $\mathcal{C}_{k}$ has only one element. Now (3.10) follows once we have proved that

$$
\prod_{\substack{i, j \in \mathcal{C}_{k} \\ i \neq j}}\left(\mathbf{m}_{i}-\mathbf{m}_{j}\right)_{\mathfrak{p}} \subseteq \mathfrak{d}_{k, \mathfrak{p}} \quad \text { for } k=1, \ldots, u,
$$

where $\mathfrak{d}_{k, \mathfrak{p}}:=\mathfrak{d}_{k} R_{\mathfrak{p}}$. Put $r:=\left[M_{k}: K\right]$, let $\alpha \mapsto \alpha^{(i)}(i=1, \ldots, r)$ denote the distinct $K$-isomorphisms of $M_{k}$ and for $\mathbf{a} \in M_{k}^{n}$ define $\mathbf{a}^{(i)}$ by applying $\alpha \mapsto \alpha^{(i)}$ to the coordinates of $\mathbf{a}$. Then there is a vector $\mathbf{m} \in R_{k, \mathfrak{p}}^{n}$ such that the left-hand side of (3.11) is equal to

$$
\prod_{1 \leq i<j \leq r}\left(\mathbf{m}^{(i)}-\mathbf{m}^{(j)}\right)_{\mathfrak{p}}^{2}=: \mathfrak{a}
$$

Put $K(\mathbf{X}):=K\left(X_{1}, \ldots, X_{n}\right), M_{k}(\mathbf{X}):=M_{k}\left(X_{1}, \ldots, X_{n}\right), L(\mathbf{X}):=$ $L\left(X_{1}, \ldots, X_{n}\right)$ where $X_{1}, \ldots, X_{n}$ are independent variables. Consider in $L(\mathbf{X})$ the polynomials

$$
A^{(i)}(\mathbf{X})=\sum_{l=1}^{n} m_{l}^{(i)} X_{l} \quad \text { for } i=1, \ldots, r,
$$

where $\left(m_{1}, \ldots, m_{n}\right)^{T}=\mathbf{m}$. Consider also the polynomial

$$
D(\mathbf{X})=\prod_{1 \leq i<j \leq r}\left\{A^{(i)}(\mathbf{X})-A^{(j)}(\mathbf{X})\right\}^{2} .
$$

By Gauss' lemma, the $R_{\mathfrak{p}}$-ideal generated by the coefficients of $D$ is equal to a. Since $R_{\mathfrak{p}}$ is a principal ideal domain, $\widehat{R}_{k, \mathfrak{p}}$ has an $R_{\mathfrak{p}}$-basis, say $\left\{\omega_{1}, \ldots, \omega_{r}\right\}$ (cf. e.g. [20], Ch. V, $\S 4$ ). Then this basis is also an $R_{\mathfrak{p}}[\mathbf{X}]$-basis of $\widehat{R}_{k, \mathfrak{p}}[\mathbf{X}]$, where $R_{\mathfrak{p}}[\mathbf{X}]:=R_{\mathfrak{p}}\left[X_{1}, \ldots, X_{n}\right]$ and $\widehat{R}_{k, \mathfrak{p}}[\mathbf{X}]:=\widehat{R}_{k, \mathfrak{p}}\left[X_{1}, \ldots\right.$ $\left.\ldots, X_{n}\right]$. Then $A \in \widehat{R}_{k, \mathfrak{p}}[\mathbf{X}]$. Further, $D(\mathbf{X})$ is precisely the discriminant of $\left\{1, A, \ldots, A^{r-1}\right\}$ with respect to $M_{k}(\mathbf{X}) / K(\mathbf{X})$ :

$$
D(\mathbf{X})=D_{M_{k}(\mathbf{X}) / K(\mathbf{X})}\left(1, A, \ldots, A^{r-1}\right) .
$$

From elementary properties of discriminants it follows that there is a polynomial $G(\mathbf{X}) \in R_{\mathfrak{p}}[\mathbf{X}]$ such that

$$
D(\mathbf{X})=G(\mathbf{X}) D_{M_{k}(\mathbf{X}) / K(\mathbf{X})}\left(\omega_{1}, \ldots, \omega_{r}\right)=G(\mathbf{X}) D_{M_{k} / K}\left(\omega_{1}, \ldots, \omega_{r}\right) .
$$

This implies that each coefficient of $D(\mathbf{X})$ is divisible in $R_{\mathfrak{p}}$ by $D_{M_{k} / K}\left(\omega_{1}, \ldots, \omega_{r}\right)$ and hence by the relative discriminant $\mathfrak{d}_{k, \mathfrak{p}}$. Therefore, $\mathfrak{a}$ is divisible by $\mathfrak{d}_{k, \mathfrak{p}}$. This proves (3.11).

4. Preliminaries. In this section we provide some basic tools needed in the proofs of our results. As before, $K$ is an algebraic number field of degree $d, S$ a finite set of prime ideals of $O_{K}$, and $O_{S}$ the ring of $S$-integers. The $O_{S}$-ideal or $O_{S}$-lattice generated by $\alpha_{1}, \ldots, \alpha_{n}$ is denoted by $\left(\alpha_{1}, \ldots, \alpha_{n}\right)$. 
We recall that if $\mathfrak{a}$ is any $O_{S}$-ideal then $|\mathfrak{a}|_{S}=N_{K / \mathbb{Q}}\left(\mathfrak{a}^{*}\right)^{1 / d}$, where $\mathfrak{a}^{*}$ is the $O_{K}$-ideal composed of prime ideals outside $S$ such that $\mathfrak{a}=\mathfrak{a}^{*} O_{S}$. We put

$$
|\alpha|_{S}=|(\alpha)|_{S} \quad \text { for } \alpha \in K \text {. }
$$

We recall that if $\mathfrak{a}=\mathfrak{b} \cdot \mathfrak{c}^{-1}$ where $\mathfrak{b}, \mathfrak{c}$ are integral $O_{S}$-ideals with $\mathfrak{b}+\mathfrak{c}=(1)$, then $m_{S}(\mathfrak{a}):=|\mathfrak{b}|_{S} \cdot|\mathfrak{c}|_{S}$. We put

$$
m_{S}(\alpha)=m_{S}((\alpha)) \quad \text { for } \alpha \in K \text {. }
$$

We shall frequently use the fact that for any two $O_{S}$-ideals $\mathfrak{a}, \mathfrak{b}$, and $k \in \mathbb{Z}$

$$
|\mathfrak{a} \mathfrak{b}|_{S}=|\mathfrak{a}|_{S} \cdot|\mathfrak{b}|_{S}, \quad m_{S}(\mathfrak{a} \mathfrak{b}) \leq m_{S}(\mathfrak{a}) m_{S}(\mathfrak{b}), \quad m_{S}\left(\mathfrak{a}^{k}\right)=m_{S}(\mathfrak{a})^{|k|},
$$

and for any two $\alpha, \beta \in K^{*}$

$$
|\alpha \beta|_{S}=|\alpha|_{S}|\beta|_{S}, \quad m_{S}(\alpha \beta) \leq m_{S}(\alpha) m_{S}(\beta), \quad m_{S}\left(\alpha^{k}\right)=m_{S}(\alpha)^{|k|} .
$$

Further, if $L / K$ is a finite extension and $T$ is the set of prime ideals of $O_{L}\left({ }^{2}\right)$ lying above those in $S$ then $O_{T}$, the ring of $T$-integers in $L$, is the integral closure of $O_{S}$ in $L$. Then we have, by the definition of $|\cdot|_{S}$ and $m_{S}(\cdot)$

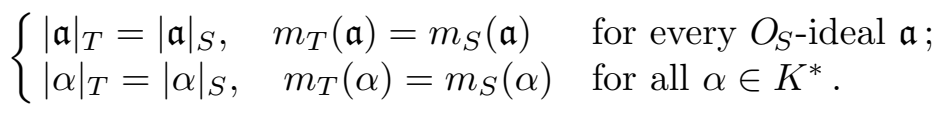

We recall that the height $h(F)$ of a polynomial $F$ with algebraic coefficients is defined as the maximum of the heights of the coefficients of $F$. Further, the height $h(\mathbf{a})$ of a vector a with algebraic coordinates is defined as the maximum of the heights of the coordinates of a. We define the height $h(A)$ of a matrix $A$ with algebraic entries in a similar way. We recall some properties of the height from ([6], Lemma 1). Let $\alpha, \beta, \alpha_{1}, \ldots, \alpha_{n}$ be algebraic numbers with $\beta \neq 0$, and $f\left(X_{1}, \ldots, X_{n}\right), g(X)$ polynomials with algebraic coefficients. Then the following properties hold:

$$
\begin{gathered}
\left\{\begin{array}{l}
h\left(\alpha^{k}\right)=h(\alpha)^{|k|} \quad \text { for } k \in \mathbb{Z} ; \\
h(\alpha \beta) \leq h(\alpha) h(\beta) ; h(\alpha / \beta) \leq h(\alpha) h(\beta) ; \\
h\left(\alpha_{1}+\ldots+\alpha_{n}\right) \leq n h\left(\alpha_{1}\right) \ldots h\left(\alpha_{n}\right) ;
\end{array}\right. \\
\left\{\begin{array}{l}
\text { if } f\left(X_{1}, \ldots, X_{n}\right) \text { has exactly } r \text { non-zero coefficients } \\
\text { and degree } d_{j} \text { in } X_{j} \text { for } j=1, \ldots, n \text { then } \\
h\left(f\left(\alpha_{1}, \ldots, \alpha_{n}\right)\right) \leq r h(f)^{r} h\left(\alpha_{1}\right)^{d_{1}} \ldots h\left(\alpha_{n}\right)^{d_{n}} ;
\end{array}\right. \\
\qquad|\alpha|_{S} \leq h(\alpha) \quad \text { if } \alpha \in K ; \\
\text { if } \theta \text { is a zero of } g(X), \text { then } h(\theta) \leq\{4 h(g)\}^{\operatorname{deg}(g)+1} .
\end{gathered}
$$

From (4.4) and (4.5) it follows that if upper bounds for the heights of algebraic numbers $\alpha_{1}, \ldots, \alpha_{n}$ are known and $\beta$ is some rational expression in $\alpha_{1}, \ldots, \alpha_{n}$, then an upper bound for $h(\beta)$ can be computed. This fact will be used frequently without refering to (4.4) and (4.5).

$\left({ }^{2}\right)$ For any algebraic number field $M$, we denote by $O_{M}$ the ring of integers of $M$. 
In what follows, let $s$ denote the cardinality of $S$, and $P$ the largest of the prime numbers lying below the prime ideals in $S$, with $P=1$ if $s=0$.

Lemma 3. Let $\mathfrak{a}$ be an $O_{S}$-ideal. Then there is an $\alpha \in \mathfrak{a}$ with $\alpha \neq 0$ and

(i) $|\alpha|_{S} \leq c_{1}|\mathfrak{a}|_{S}$

(ii) $h(\alpha) \leq c_{1}|\mathfrak{a}|_{S}$ if $\mathfrak{a}$ is integral

where $c_{1}$ is an effectively computable number depending only on $d$ and $\left|D_{K}\right|$.

Proof. First we prove (ii). Let $\mathfrak{a}$ be an integral $O_{S^{-}}$-ideal and let $\mathfrak{a}^{*}$ be the $O_{K}$-ideal composed of prime ideals outside $S$ such that $\mathfrak{a}=\mathfrak{a}^{*} O_{S}$. Let $\alpha \mapsto \alpha^{(i)}$ be the distinct $\mathbb{Q}$-isomorphisms of $K$ in $\overline{\mathbb{Q}}$. By Satz 6 of [16], $\mathfrak{a}^{*}$ contains an element $\alpha \neq 0$ with $\left|\alpha^{(i)}\right| \leq c_{1} N_{K / \mathbb{Q}}\left(\mathfrak{a}^{*}\right)^{1 / d}$ for $i=1, \ldots, d$ where $c_{1}$ is an effectively computable number depending only on $d$ and $\left|D_{K}\right|$. Now Lemma 3(ii) follows from the fact that $h(\alpha) \leq \max _{i}\left|\alpha^{(i)}\right|$ and $N_{K / \mathbb{Q}}\left(\mathfrak{a}^{*}\right)^{1 / d}=|\mathfrak{a}|_{S}$

We now prove (i). Take $\delta \in K^{*}$ such that $\mathfrak{a}^{\prime}:=\delta \mathfrak{a} \subseteq O_{S}$. By (ii) there is an $\alpha^{\prime} \in \mathfrak{a}^{\prime}$ such that $\alpha^{\prime} \neq 0$ and $h\left(\alpha^{\prime}\right) \leq c_{1}\left|\mathfrak{a}^{\prime}\right|_{S}$. Now (4.6) implies that $\left|\alpha^{\prime}\right|_{S} \leq c_{1}\left|\mathfrak{a}^{\prime}\right|_{S}$. Put $\alpha:=\delta^{-1} \alpha^{\prime}$. Then (4.1), (4.2) imply that $|\alpha|_{S} \leq c_{1}|\mathfrak{a}|_{S}$.

We write $\alpha \equiv \beta \bmod \mathfrak{a}$ if $\alpha-\beta$ belongs to the $O_{S}$-ideal $\mathfrak{a}$ and, for $\gamma \in O_{S}$, $\alpha \equiv \beta \bmod \gamma$ if $\alpha-\beta \in(\gamma)$.

Lemma 4. Let $\mathfrak{a}$ be an integral $O_{S}$-ideal and $\beta \in O_{S}$. Then there is an $\alpha \in O_{K}$ such that

$$
\alpha \equiv \beta \bmod \mathfrak{a}, \quad h(\alpha) \leq c_{2}|\mathfrak{a}|_{S},
$$

where $c_{2}$ is an effectively computable number depending only on $d$ and $\left|D_{K}\right|$.

Proof. See Lemma 6 of [6] with an explicitly given $c_{2}$.

We now prove the result stated in Section 2, that every non-principal $O_{S}$-ideal class contains an integral ideal with generators of small height.

Lemma 5. Let $\mathfrak{a}$ be a non-principal $O_{S}$-ideal. Then there are $\gamma \in K^{*}$ and $\alpha, \beta \in O_{S}$ such that

$$
\gamma \mathfrak{a}=(\alpha, \beta), \quad h(\alpha) \leq c_{3}, \quad h(\beta) \leq c_{3},
$$

where $c_{3}$ is an effectively computable number depending only on $d$ and $\left|D_{K}\right|$.

Proof. $c_{4}, c_{5}$ will denote effectively computable numbers depending only on $d$ and $\left|D_{K}\right|$. By Lemma 3(i) we can choose $\gamma \in \mathfrak{a}^{-1}$ such that $\gamma \neq 0$ and $|\gamma|_{S} \leq c_{1}|\mathfrak{a}|_{S}^{-1}$. Put $\mathfrak{b}=\gamma \mathfrak{a}$. Note that $\mathfrak{b}$ is an integral $O_{S}$-ideal with $|\mathfrak{b}|_{S} \leq c_{1}$. By Lemma 3(ii) we can choose $\alpha \in \mathfrak{b}$ such that $\alpha \neq 0$ and $h(\alpha) \leq c_{1}|\mathfrak{b}|_{S} \leq c_{4}$. Then $\operatorname{ord}_{\mathfrak{p}}(\alpha) \geq \operatorname{ord}_{\mathfrak{p}}(\mathfrak{b})$ for all $\mathfrak{p} \in M_{K} \backslash S$. By the Chinese Remainder Theorem (see e.g. [2], Ch. III, §3, Thm. 4) we can 
choose $\beta \in O_{S}$ such that

$$
\begin{aligned}
& \operatorname{ord}_{\mathfrak{p}}(\beta)=\operatorname{ord}_{\mathfrak{p}}(\mathfrak{b}) \quad \text { for all } \mathfrak{p} \in M_{K} \backslash S \text { with } \operatorname{ord}_{\mathfrak{p}}(\alpha)>\operatorname{ord}_{\mathfrak{p}}(\mathfrak{b}), \\
& \operatorname{ord}_{\mathfrak{p}}(\beta) \geq \operatorname{ord}_{\mathfrak{p}}(\mathfrak{b}) \quad \text { for all } \mathfrak{p} \in M_{K} \backslash S \text { with } \operatorname{ord}_{\mathfrak{p}}(\alpha)=\operatorname{ord}_{\mathfrak{p}}(\mathfrak{b}) .
\end{aligned}
$$

Further, if $\beta$ satisfies (4.8) then so does every $\beta^{\prime} \in O_{S}$ with $\beta^{\prime} \equiv \beta \bmod \alpha$. Hence by Lemma 4 and (4.6) there is a $\beta \in O_{S}$ satisfying (4.8) and

$$
h(\beta) \leq c_{2}|\alpha|_{S} \leq c_{2} h(\alpha) \leq c_{5} .
$$

For every $\mathfrak{p} \in M_{K} \backslash S$ we have $\operatorname{ord}_{\mathfrak{p}}(\mathfrak{b})=\min \left(\operatorname{ord}_{\mathfrak{p}}(\alpha), \operatorname{ord}_{\mathfrak{p}}(\beta)\right)$. Hence $\mathfrak{b}=(\alpha, \beta)$. This proves Lemma 5 .

We now state some results on $S$-units.

Lemma 6. Let $\alpha \in K^{*}$ and $n \in \mathbb{N}$. Then there is an $\varepsilon \in O_{S}^{*}$ such that

$$
h\left(\varepsilon^{n} \alpha\right) \leq c_{6}^{n} m_{S}(\alpha), \quad \text { and } \quad h\left(\varepsilon^{n} \alpha\right) \leq c_{6}^{n}|\alpha|_{S} \quad \text { if } \alpha \in O_{S} \backslash\{0\},
$$

where $c_{6}$ is an effectively computable number depending only on $d,\left|D_{K}\right|, s$ and $P$.

Proof. $c_{7}, c_{8}$ will denote effectively computable numbers depending only on $d,\left|D_{K}\right|, s$ and $P$. Let $\mathfrak{a}, \mathfrak{b}$ be the integral $O_{S}$-ideals with $(\alpha)=\mathfrak{a b}^{-1}$ and $\mathfrak{a}+\mathfrak{b}=(1)$. By Lemma 3(i) we can choose $\gamma \in \mathfrak{b}$ with $\gamma \neq 0$ and $|\gamma|_{S} \leq c_{1}|\mathfrak{b}|_{S}$. Put $\beta=\alpha \cdot \gamma$. Then $\beta \in \mathfrak{a}$ and $|\beta|_{S} \leq c_{1}|\mathfrak{a}|_{S}$. Note that $\beta, \gamma \in O_{S}$. By Lemma 10 of [6] there are $\eta, \zeta \in O_{S}^{*}$ such that

$$
h\left(\eta^{n} \beta\right) \leq c_{7}^{n}|\beta|_{S} \leq c_{8}^{n}|\mathfrak{a}|_{S}, \quad h\left(\zeta^{n} \gamma\right) \leq c_{7}^{n}|\gamma|_{S} \leq c_{8}^{n}|\mathfrak{b}|_{S} .
$$

Put $\varepsilon=\eta / \zeta$. Then, by Lemma $3, h\left(\varepsilon^{n} \alpha\right) \leq c_{6}^{n}|\mathfrak{a}|_{S}|\mathfrak{b}|_{S}=c_{6}^{n} m_{S}(\alpha)$. Further, if $\alpha \in O_{S} \backslash\{0\}$ then $m_{S}(\alpha)=|\alpha|_{S}$ and the proof is complete.

We apply Lemma 6 in the following situation. Let $L / K$ be a finite, normal extension, and let $A_{1}, \ldots, A_{t}$ be finite, non-empty subsets of $L^{*}$ such that

$$
\sigma\left(A_{i}\right)=A_{\sigma(i)} \quad \text { for } i=1, \ldots, t, \quad \sigma \in \operatorname{Gal}(L / K),
$$

where $(\sigma(1), \ldots, \sigma(t))$ is a permutation of $(1, \ldots, t)$ for $\sigma \in \operatorname{Gal}(L / K)$. Consider the $\operatorname{Gal}(L / K)$-orbits $\mathcal{C}_{1}, \ldots, \mathcal{C}_{u}$ of $\{1, \ldots, t\}$ introduced in Section 3 (where $i, j$ belong to the same orbit if and only if $\sigma(i)=j$ for some $\sigma \in \operatorname{Gal}(L / K))$. Let $T$ be the set of prime ideals in $O_{L}$ lying above those in $S$. Assume that

$$
\begin{array}{ll}
m_{T}(\alpha) \leq C & \text { for } \alpha \in A_{1} \cup \ldots \cup A_{t} \quad \text { and } \\
h(\alpha / \beta) \leq C & \text { for } \alpha, \beta \in A_{i}, i=1, \ldots, t .
\end{array}
$$

Lemma 7. For every $n_{1}, \ldots, n_{u} \in \mathbb{Z} \backslash\{0\}$ there are $\varepsilon_{1}, \ldots, \varepsilon_{t} \in O_{T}^{*}$ such that

$$
\sigma\left(\varepsilon_{i}\right)=\varepsilon_{\sigma(i)} \quad \text { for } i=1, \ldots, t \text { and for each } \sigma \in \operatorname{Gal}(L / K)
$$


and

$$
h\left(\varepsilon_{i}^{n_{j}} \alpha\right) \leq c_{9}^{\left|n_{j}\right|} C^{2} \quad \text { for all } \alpha \in A_{i} \text { with } i \in \mathcal{C}_{j}, j=1, \ldots, u,
$$

where $c_{9}$ is an effectively computable number depending only on $d,[L: K]$, $\left|D_{L}\right|, s$ and $P$.

Proof. Let $\mathcal{C}$ be an arbitrary but fixed $\operatorname{Gal}(L / K)$-orbit of $\{1, \ldots, t\}$. It suffices to prove that for every $n \in \mathbb{N}$, there are $\varepsilon_{i} \in O_{T}^{*}$ with $i \in \mathcal{C}$ such that

$$
\sigma\left(\varepsilon_{i}\right)=\varepsilon_{\sigma(i)} \quad \text { for each } i \in \mathcal{C} \text { and } \sigma \in \operatorname{Gal}(L / K)
$$

and

$$
h\left(\varepsilon_{i}^{n} \alpha\right) \leq c_{9}^{n} C^{2} \quad \text { for all } \alpha \in A_{i} \text { with } i \in \mathcal{C} .
$$

Let $a_{i}$ be the cardinality of $A_{i}$ for $i \in \mathcal{C}$. Put $\beta_{i}=\prod_{\alpha \in A_{i}} \alpha$ for $i \in \mathcal{C}$. Then by (4.9) we have $\sigma\left(\beta_{i}\right)=\beta_{\sigma(i)}$ for each $i \in \mathcal{C}$ and $\sigma \in \operatorname{Gal}(L / K)$. Let $M_{i}$ $(i \in \mathcal{C})$ be the field defined by

$$
\operatorname{Gal}\left(L / M_{i}\right)=\{\sigma \in \operatorname{Gal}(L / K): \sigma(i)=i\} .
$$

Then $\beta_{i} \in M_{i}^{*}$ for each $i \in \mathcal{C}$. By (4.9), Lemma 6, (3.4) and (4.10), we can choose $\varepsilon_{i} \in O_{T}^{*} \cap M_{i}^{*}(i \in \mathcal{C})$ such that $(4.12)$ is satisfied and

$$
h\left(\varepsilon_{i}^{n a_{i}} \beta_{i}\right) \leq c_{10}^{n a_{i}} m_{T}\left(\beta_{i}\right) \leq\left\{c_{9}^{n} C\right\}^{a_{i}}
$$

with some effectively computable number $c_{10}$ which depends only on $d$, $[L: K],\left|D_{L}\right|, s$ and $P$. By the second inequality of (4.10), for every $\alpha \in A_{i}$ we have

$$
h\left(\alpha^{a_{i}} / \beta_{i}\right) \leq \prod_{\beta \in A_{i}} h(\alpha / \beta) \leq C^{a_{i}} .
$$

By combining this with (4.13) we get

$$
h\left(\varepsilon_{i}^{n} \alpha\right) \leq\left\{h\left(\varepsilon_{i}^{n a_{i}} \beta_{i}\right) h\left(\alpha^{a_{i}} / \beta_{i}\right)\right\}^{1 / a_{i}} \leq c_{9}^{n} C^{2} \quad \text { for } i \in \mathcal{C} .
$$

The next result is our main tool in the proof of Theorem 2 .

Lemma 8. Let $x_{0}, x_{1}, x_{2} \in K^{*}$ such that

$$
x_{0}+x_{1}+x_{2}=0 \quad \text { and } \quad m_{S}\left(x_{i}\right) \leq A \quad \text { for } i=0,1,2 .
$$

Then

$$
h\left(x_{i} / x_{j}\right) \leq c_{11} A^{c_{12}} \quad \text { for } i, j \in\{0,1,2\},
$$

where $c_{11}, c_{12}$ are effectively computable numbers depending only on $d,\left|D_{K}\right|$, $s$ and $P$.

Proof. Let $\mathfrak{a}_{i}, \mathfrak{b}_{i}$ be the integral $O_{S^{-}}$-ideals such that $\left(x_{i}\right)=\mathfrak{a}_{i} \mathfrak{b}_{i}^{-1}$ and $\mathfrak{a}_{i}+\mathfrak{b}_{i}=(1)(i=0,1,2)$ and put $\mathfrak{b}=\mathfrak{b}_{1} \mathfrak{b}_{2} \mathfrak{b}_{3}$. By Lemma 3 (i) we can choose $\beta \in \mathfrak{b}$ such that $\beta \neq 0$ and $|\beta|_{S} \leq c_{1}|\mathfrak{b}|_{S}$. Put $y_{i}=\beta x_{i}$ for $i=0,1,2$. Then

$$
y_{0}+y_{1}+y_{2}=0, \quad y_{i} \in O_{S} \backslash\{0\},\left|y_{i}\right|_{S} \leq B:=c_{1} A^{4} \text { for } i=0,1,2 .
$$


By Lemma 11 of [6], there are effectively computable numbers $c_{13}, c_{14}$ depending only on $d,\left|D_{K}\right|, s$ and $P$, such that

$$
h\left(y_{i} / y_{j}\right) \leq c_{13} B^{c_{14}} \quad \text { for } i, j \in\{0,1,2\} .
$$

This implies (4.14). We remark that Lemma 11 of [6] was a reformulation of an effective result of Györy on $S$-unit equations ([10], Lemma 6), and that Györy proved this result by applying Baker's theory on linear forms in logarithms and its $p$-adic analogue.

5. Effective reduction of matrices. As before, let $K$ be an algebraic number field and $S$ a finite set of prime ideals of $O_{K}$. The parameters $d$, $D_{K}, s$ and $P$ have the same meaning as in the previous sections. If $V$ is any set then $V^{m, n}$ denotes the collection of $m \times n$ matrices with entries in $V$. If $A, B \in K^{m, n}$ then we write $A \equiv B \bmod \mathfrak{a}$ if the entries of $A-B$ belong to the $O_{S}$-ideal $\mathfrak{a}$, and $A \equiv B \bmod \gamma$ if $\mathfrak{a}=(\gamma)$ for some $\gamma \in K^{*}$. For every integral $O_{S}$-ideal $\mathfrak{a}$, let $G(n, \mathfrak{a})$ be the multiplicative group of matrices $U$ with the following properties:

$$
\begin{gathered}
U \in O_{S}^{n, n} ; \quad \operatorname{det} U \in O_{S}^{*} ; \\
U \equiv\left(\begin{array}{ccc}
\varepsilon_{1} & & 0 \\
& \ddots & \\
0 & & \varepsilon_{n}
\end{array}\right) \bmod \mathfrak{a} \quad \text { for some } \varepsilon_{1}, \ldots, \varepsilon_{n} \in O_{S}^{*} .
\end{gathered}
$$

It easily follows from (1.5) that if $\mathfrak{a}=(\alpha, \beta)$ and $\mathfrak{M}$ is the $O_{S}$-lattice $\left(\mathbf{e}_{1}, \ldots, \mathbf{e}_{n-1}, \alpha \mathbf{e}_{n}, \beta \mathbf{e}_{n}\right)$ then

$$
G(n, \mathfrak{a}) \subseteq G(\mathfrak{M}) .
$$

In this section we shall prove the following result.

Lemma 9. Let $n \geq 2$ be an integer. For every non-singular matrix $A$ in $O_{S}^{n, n}$, there is a matrix $U$ in $G(n, \mathfrak{a})$ such that

$$
h(A U) \leq c_{15}\left\{|\operatorname{det} A|_{S} \cdot|\mathfrak{a}|_{S}\right\}^{c_{15}^{\prime}}
$$

where $c_{15}, c_{15}^{\prime}$ are effectively computable numbers such that $c_{15}$ depends only on $d,\left|D_{K}\right|, s, P$ and $n$, and $c_{15}^{\prime}$ only on $n$.

In the proof of Lemma 9 we need some auxiliary results.

LEMMA 10. Let $A_{1}, A_{2} \in O_{S}^{n, n}$ be two non-singular matrices with $\left(\operatorname{det} A_{1}\right) /\left(\operatorname{det} A_{2}\right) \in O_{S}^{*}$ and $A_{1} \equiv A_{2} \bmod \left(\operatorname{det} A_{1}\right) \mathfrak{a}$. Then there is a matrix $U \in G(n, \mathfrak{a})$ with $A_{2}=A_{1} U$.

Proof. Let $U=A_{1}^{-1} A_{2}$. By assumption, there is a matrix $C \in \mathfrak{a}^{n, n}$ such that $A_{2}=A_{1}+\left(\operatorname{det} A_{1}\right) C$. Hence

$$
U=A_{1}^{-1}\left(A_{1}+\left(\operatorname{det} A_{1}\right) C\right)=I+\left\{\left(\operatorname{det} A_{1}\right) A_{1}^{-1}\right\} C \equiv I \bmod \mathfrak{a},
$$


where $I$ is the $n \times n$ identity matrix. Further, $\operatorname{det} U \in O_{S}^{*}$. Hence $U \in$ $G(n, \mathfrak{a})$.

LEMmA 11. Let $A \in K^{m, n}$ be a matrix of rank $m, \mathbf{b} \in K^{m}$ and assume that the system of linear equations

$$
A \mathbf{x}=\mathbf{b} \quad \text { in } \mathbf{x} \in O_{S}^{n}
$$

is solvable. Then (5.2) has a solution $\mathbf{x} \in O_{S}^{n}$ with

$$
h(\mathbf{x}) \leq c_{16}\{h(A) h(\mathbf{b})\}^{c_{16}^{\prime}},
$$

where $c_{16}, c_{16}^{\prime}$ are effectively computable numbers such that $c_{16}$ depends only on $d,\left|D_{K}\right|$ and $n$, and $c_{16}^{\prime}$ only on $n$.

Proof. For $i=17, \ldots, 20, c_{i}, c_{i}^{\prime}$ will denote effectively computable numbers such that $c_{i}$ depends only on $d,\left|D_{K}\right|$ and $n$, and $c_{i}^{\prime}$ only on $n$. We assume that the matrix $C$ formed by the first $m$ columns of $A$ is non-singular, which is no restriction. Then

$$
C^{-1} A=\left(I, A^{\prime}\right)
$$

where $I$ is the $m \times m$ unit matrix and $A^{\prime} \in K^{m, n-m}$. For every solution $\mathbf{x} \in O_{S}^{n}$ of (5.2), let $\mathbf{y}, \mathbf{z}$ be the vectors consisting of the first $m$ coordinates of $\mathbf{x}$ and the last $n-m$ coordinates of $\mathbf{x}$, respectively. Put $\mathbf{b}^{\prime}=C^{-1} \mathbf{b}$. Then (5.2) is equivalent to

$$
\mathbf{y}+A^{\prime} \mathbf{z}=\mathbf{b}^{\prime} \quad \text { in } \mathbf{y} \in O_{S}^{m}, \mathbf{z} \in O_{S}^{n-m} .
$$

Let $\left(\mathbf{y}_{0}, \mathbf{z}_{0}\right)$ be a solution of (5.3). By (2.1), (4.4) and (4.6), there is a non-zero rational integer $a$ such that $a A^{\prime}$ has integral entries in $K$ and

$$
|a|_{S} \leq c_{17} h\left(A^{\prime}\right)^{c_{17}^{\prime}} \leq c_{18} h(A)^{c_{18}^{\prime}} .
$$

By Lemma 4 , there is a vector $\mathbf{z} \in O_{S}^{n-m}$ with

$$
\mathbf{z} \equiv \mathbf{z}_{0} \bmod a, \quad h(\mathbf{z}) \leq c_{2}|a|_{S} \leq c_{19} h(A)^{c_{19}^{\prime}} .
$$

Put $\mathbf{y}=\mathbf{b}^{\prime}-A^{\prime} \mathbf{z}$. It is easy to see that $\mathbf{y} \in O_{S}^{m}$. Further, $(\mathbf{y}, \mathbf{z})$ is a solution of (5.3), and, by (4.4) and (5.4),

$$
h(\mathbf{y}) \leq c_{20}\left\{h\left(A^{\prime}\right) h(\mathbf{z}) h(\mathbf{b})\right\}^{c_{20}^{\prime}} \leq c_{16}\{h(A) h(\mathbf{b})\}^{c_{16}^{\prime}} .
$$

If $B$ is any $n \times n$ matrix then we denote by $B_{i j}$ the matrix obtained by removing the $i$ th row and $j$ th column from $B$. For $n=1$, we shall take $\operatorname{det} B_{1,1}=1$. If $\mathfrak{p}$ is any prime ideal of $O_{K}$ outside $S$ and $\alpha_{1}, \ldots, \alpha_{n} \in K$ then we put $\operatorname{ord}_{\mathfrak{p}}\left(\alpha_{1}, \ldots, \alpha_{n}\right)=\min \left(\operatorname{ord}_{\mathfrak{p}}\left(\alpha_{1}\right), \ldots, \operatorname{ord}_{\mathfrak{p}}\left(\alpha_{n}\right)\right)$.

Lemma 12. Let $n \geq 1$ be an integer, let $A \in O_{S}^{n, n}$ be a non-singular matrix, let $S^{\prime}$ be a finite set of prime ideals of $O_{K}$ outside $S$, and let $\mathfrak{b}$ be 
an $O_{S}$-ideal with

$$
\begin{aligned}
& \operatorname{ord}_{\mathfrak{p}}(\mathfrak{b})=0 \quad \text { for all } \mathfrak{p} \in M_{K} \backslash\left(S \cup S^{\prime}\right), \\
& \operatorname{ord}_{\mathfrak{p}}(\mathfrak{b})>\operatorname{ord}_{\mathfrak{p}}(\operatorname{det} A) \quad \text { for all } \mathfrak{p} \in S^{\prime} .
\end{aligned}
$$

Then there exists a matrix $B \in O_{S}^{n, n}$ with the following properties:

$$
\begin{gathered}
B \equiv A \bmod \mathfrak{b} ; \\
h(B) \leq c_{21}|\mathfrak{b}|_{S}^{c_{21}},
\end{gathered}
$$

where $c_{21}, c_{21}^{\prime}$ are effectively computable numbers such that $c_{21}$ depends only on $d,\left|D_{K}\right|$ and $n$, and $c_{21}^{\prime}$ only on $n$;

$$
\begin{gathered}
\operatorname{det} B \neq 0 ; \\
\operatorname{ord}_{\mathfrak{p}}\left(\operatorname{det} B_{1 n}, \ldots, \operatorname{det} B_{n n}\right)=0 \quad \text { for all } \mathfrak{p} \in M_{K} \backslash\left(S \cup S^{\prime}\right) .
\end{gathered}
$$

Proof. $c_{22}, \ldots, c_{27}$ will denote effectively computable numbers of the form $c|\mathfrak{b}|_{S}^{c^{\prime}}$, where $c$ depends only on $d,\left|D_{K}\right|, n$, and $c^{\prime}$ only on $n$. We proceed by induction on $n$. For $n=1$, our assertion means that if $\alpha \in O_{S} \backslash\{0\}$ and $\mathfrak{b}$ is an integral $O_{S}$-ideal with $\operatorname{ord}_{\mathfrak{p}}(\mathfrak{b})=0$ for all $\mathfrak{p} \in M_{K} \backslash\left\{S \cup S^{\prime}\right\}$ and $\operatorname{ord}_{\mathfrak{p}}(\mathfrak{b})>\operatorname{ord}_{\mathfrak{p}}(\alpha)$ for all $\mathfrak{p} \in S^{\prime}$, then there is a $\beta \in O_{S} \backslash\{0\}$ with $\beta \equiv \alpha \bmod \mathfrak{b}$ and $h(\beta) \leq c_{22}$; by Lemma 4 we know that this is true. Hence let $n \geq 2$ and assume that Lemma 12 holds for $n-1$.

By Lemma 4, there is a matrix $A^{\prime} \in O_{S}^{n, n}$ such that

$$
A^{\prime} \equiv A \bmod \mathfrak{b} \quad \text { and } \quad h\left(A^{\prime}\right) \leq c_{23} .
$$

Then $\operatorname{det} A^{\prime} \equiv \operatorname{det} A \bmod \mathfrak{b}$. Since $\operatorname{ord}_{\mathfrak{p}}(\mathfrak{b})>\operatorname{ord} \mathfrak{p}(\operatorname{det} A)$ for all $\mathfrak{p} \in S^{\prime}$, this implies that $\operatorname{det} A^{\prime} \neq 0$. Hence at least one of the $\operatorname{determinants} \operatorname{det}\left(A_{1, n}^{\prime}\right), \ldots$ $\ldots, \operatorname{det}\left(A_{n, n}^{\prime}\right)$ must be non-zero; we assume that $\operatorname{det}\left(A_{n, n}\right) \neq 0$, which is no restriction. Put $A=A_{n, n}^{\prime}$. Since $h(\bar{A}) \leq h\left(A^{\prime}\right) \leq c_{23}$, by (4.6) we have $|\operatorname{det} \bar{A}|_{S} \leq h(\bar{A}) \leq c_{23}$. It is easy to see that there is an integral $O_{S}$-ideal $\overline{\mathfrak{b}}$ such that

$$
\begin{aligned}
\operatorname{ord}_{\mathfrak{p}}(\overline{\mathfrak{b}}) & \geq \operatorname{ord}_{\mathfrak{p}}(\mathfrak{b}) ; \\
\operatorname{ord}_{\mathfrak{p}}(\overline{\mathfrak{b}}) & >\max \left(\operatorname{ord}_{\mathfrak{p}}(\operatorname{det} \bar{A}), \operatorname{ord}_{\mathfrak{p}}\left(\operatorname{det} A^{\prime}\right)\right) \quad \text { for } \mathfrak{p} \in S^{\prime}, \\
\operatorname{ord}_{\mathfrak{p}}(\overline{\mathfrak{b}}) & \geq 0 \quad \text { for all } \mathfrak{p} \in M_{K} \backslash\left(S \cup S^{\prime}\right), \\
|\overline{\mathfrak{b}}|_{S} & \leq c_{24} .
\end{aligned}
$$

By the induction hypothesis, there is a matrix $\bar{B} \in O_{S}^{n-1, n-1}$ such that

$$
\begin{aligned}
\bar{B} \equiv \bar{A} \bmod \overline{\mathfrak{b}}, \quad h(\bar{B}) \leq c_{25}, \quad \operatorname{det} \bar{B} \neq 0, & \\
\operatorname{ord}_{\mathfrak{p}}\left(\operatorname{det} \bar{B}_{1, n-1}, \ldots, \operatorname{det} \bar{B}_{n-1, n-1}\right) & =0 \\
& \text { for all } \mathfrak{p} \in M_{K} \backslash\left(S \cup S^{\prime}\right) .
\end{aligned}
$$

Here we put $\bar{B}_{1, n-1}:=1$ if $n=2$. By the Chinese Remainder Theorem, we 
can choose $\xi \in O_{S}$ such that

$$
\begin{cases}\operatorname{ord}_{\mathfrak{p}}(\xi) \geq \operatorname{ord}_{\mathfrak{p}}(\overline{\mathfrak{b}}) & \text { for all } \mathfrak{p} \in S^{\prime} \\ \operatorname{ord}_{\mathfrak{p}}(\xi)=0 & \text { for all } \mathfrak{p} \in M_{K} \backslash\left(S \cup S^{\prime}\right) \\ & \text { with } \operatorname{ord}_{\mathfrak{p}}\left(\gamma_{1}, \ldots, \gamma_{n}\right)>0 ; \\ \operatorname{ord}_{\mathfrak{p}}(\xi)>0 & \text { for all } \mathfrak{p} \in M_{K} \backslash\left(S \cup S^{\prime}\right) \text { with } \operatorname{ord}_{\mathfrak{p}}\left(\gamma_{n}\right)>0 \\ & \quad \text { and ordd }\left(\gamma_{1}, \ldots, \gamma_{n-1}\right)=0 ; \\ \operatorname{ord}_{\mathfrak{p}}(\xi) \geq 0 & \text { for the other prime ideals } \mathfrak{p} \text { in } M_{K} \backslash\left(S \cup S^{\prime}\right) .\end{cases}
$$

Then, by (5.5) and (5.11), $\xi \in \mathfrak{b}$. It is easy to check that if $\xi_{0}$ satisfies (5.13), then so does every $\xi \in O_{S}$ with $\xi \equiv \xi_{0} \bmod (\operatorname{det} \bar{B}) \overline{\mathfrak{b}}$. By $(5.10)$, (5.12), (4.6) and Lemma 4, we can choose $\xi$ such that

$$
h(\xi) \leq c_{26} \text {. }
$$

Let $C$ be the $n \times n$ matrix obtained from $A^{\prime}$ by replacing $\bar{A}$ by $\bar{B}$ and leaving the $n$th row and $n$th column of $A^{\prime}$ unchanged. Let $C=\left(c_{i j}\right)_{1 \leq i, j \leq n}$. We construct $B$ from $C$ by replacing $c_{n, n-1}$ by $c_{n, n-1}+\xi$ with the above $\xi$ and leaving the other entries of $C$ unchanged. Write

$$
\bar{\beta}_{i}=\operatorname{det} \bar{B}_{i, n-1}, \quad \gamma_{i}=\operatorname{det} C_{i n}, \quad \text { beta } a_{i}=\operatorname{det} B_{i n} .
$$

Then

$$
\text { (5.15) } \beta_{1}=\gamma_{1}+\xi \bar{\beta}_{1}, \quad \ldots, \quad \beta_{n-1}=\gamma_{n-1}+\xi \bar{\beta}_{n-1}, \quad \beta_{n}=\gamma_{n}=\operatorname{det} \bar{B} .
$$

By construction, we have $B \equiv A \bmod \mathfrak{b}$, hence

$$
\operatorname{ord}_{\mathfrak{p}}(\operatorname{det} B)=\operatorname{ord}_{\mathfrak{p}}(\operatorname{det} A) \quad \text { for all } \mathfrak{p} \in S^{\prime},
$$

which implies that $\operatorname{det} B \neq 0$. Further, $h(B) \leq c_{27}$ by (5.10), (5.12) and (5.14).

It remains to prove (5.9), i.e.

$$
\operatorname{ord}_{\mathfrak{p}}\left(\beta_{1}, \ldots, \beta_{n}\right)=0 \quad \text { for all } \mathfrak{p} \in M_{K} \backslash\left(S \cup S^{\prime}\right) .
$$

This is obvious if $\operatorname{ord}_{\mathfrak{p}}\left(\beta_{n}\right)=\operatorname{ord}_{\mathfrak{p}}(\operatorname{det} \bar{B})=0$. Let $\mathfrak{p} \in M_{K} \backslash\left(S \cup S^{\prime}\right)$ be such that $\operatorname{ord}_{\mathfrak{p}}(\operatorname{det} \bar{B})>0$. If $\operatorname{ord}_{\mathfrak{p}}\left(\gamma_{i}\right)=0$ for some $i \in\{1, \ldots, n-1\}$ then by (5.15) and (5.13) we have $\operatorname{ord}_{\mathfrak{p}}\left(\beta_{i}\right)=0$. By (5.12) we know that there is an $i \in\{1, \ldots, n-1\}$ with $\operatorname{ord}_{\mathfrak{p}}\left(\bar{\beta}_{i}\right)=0$. Hence if $\operatorname{ord}_{\mathfrak{p}}\left(\gamma_{j}\right)>0$ for $j=1, \ldots, n-1$, then by $(5.15)$ and $(5.13), \operatorname{ord}_{\mathfrak{p}}\left(\beta_{i}\right)=0$. This proves $(5.16)$ and completes the proof of Lemma 12.

Proof of Lemma 9. By Lemma 3(ii), we can choose $\alpha \in \mathfrak{a}$ with $\alpha \neq 0$ and $h(\alpha) \leq c_{1}|\mathfrak{a}|_{S}$. Further, by Lemma 6 , we can choose $\varepsilon \in O_{S}^{*}$ such that $h(\varepsilon \operatorname{det} A) \leq c_{6}|\operatorname{det} A|_{S}$. Put

$$
A_{1}=A\left(\begin{array}{cccc}
\varepsilon & & & 0 \\
& 1 & & \\
& & \ddots & \\
0 & & & 1
\end{array}\right) \quad \text { and } \quad \Delta=\operatorname{det} A_{1}
$$


then

$$
h(\Delta) \leq c_{6}|\operatorname{det} A|_{S} .
$$

Since

$$
\left(\begin{array}{llll}
\varepsilon & & & 0 \\
& 1 & & \\
& & \ddots & \\
0 & & & 1
\end{array}\right) \in G(n, \mathfrak{a}),
$$

by Lemma 10 it suffices to prove that there is a matrix $C \in O_{S}^{n, n}$ with

$$
C \equiv A_{1} \bmod \alpha \Delta, \quad h(C) \leq c_{28}(h(\alpha) h(\Delta))^{c_{28}^{\prime},} \quad \operatorname{det} C=\Delta,
$$

where $c_{28}, c_{28}^{\prime}$ are effectively computable numbers such that $c_{28}$ depends only on $d,\left|D_{K}\right|, s, P$ and $n$, and $c_{28}^{\prime}$ only on $n$. In what follows, $c_{29}, \ldots, c_{32}$ denote effectively computable numbers of the form $c(h(\alpha) h(\Delta))^{c^{\prime}}$ where $c$ depends only on $d,\left|D_{K}\right|, s, P$ and $n$, and $c^{\prime}$ only on $n$; we shall frequently use the fact that, by (4.6), $|\alpha|_{S} \leq h(\alpha)$ and $|\Delta|_{S} \leq h(\Delta)$.

By Lemma 12, there is a matrix $B \in O_{S}^{n, n}$ such that

$$
\begin{gathered}
B \equiv A_{1} \bmod \alpha \Delta^{2}, \quad h(B) \leq c_{29}, \quad \operatorname{det} B \neq 0, \\
\operatorname{ord}_{\mathfrak{p}}\left(\operatorname{det} B_{1 n}, \ldots, \operatorname{det} B_{n n}\right)=0 \\
\text { for every } \mathfrak{p} \in M_{K} \backslash S \text { with } \operatorname{ord}_{\mathfrak{p}}(\alpha \Delta)=0 .
\end{gathered}
$$

Let $\kappa_{1}, \ldots, \kappa_{n}$ be the entries in the last column of $B$, and put $\Delta_{i}=\operatorname{det} B_{\text {in }}$ for $i=1, \ldots, n$. We shall construct $C$ by replacing $\kappa_{i}$ by $\kappa_{i}+\xi_{i} \alpha \Delta$ for certain $\xi_{i} \in O_{S}, i=1, \ldots, n$, and leaving the other entries of $B$ unchanged. Then, by (5.18), $C \equiv A_{1} \bmod \alpha \Delta$. We have to choose $\xi_{1}, \ldots, \xi_{n}$ such that $\operatorname{det} C=\Delta$, that is,

$$
\sum_{i=1}^{n}(-1)^{i-1} \Delta_{i}\left(\kappa_{i}+\xi_{i} \alpha \Delta\right)=\Delta .
$$

Since $B \equiv A_{1} \bmod \alpha \Delta^{2}$ and $\operatorname{det} A_{1}=\Delta$, there is a $\gamma \in O_{S}$ such that

$$
\operatorname{det} B=\Delta-\gamma \alpha \Delta^{2} \text {. }
$$

Hence

$$
\sum_{i=1}^{n}(-1)^{i-1} \Delta_{i} \kappa_{i}=\Delta-\gamma \alpha \Delta^{2}=\Delta(1-\gamma \alpha \Delta) .
$$

By inserting this into (5.19) we get

$$
\sum_{i=1}^{n}(-1)^{i-1} \Delta_{i} \xi_{i}=\gamma \Delta
$$


If $\mathfrak{p}$ is a prime ideal of $O_{K}$ outside $S$ with $\operatorname{ord}_{\mathfrak{p}}(\alpha \Delta)>0$, then $\operatorname{ord}_{\mathfrak{p}}(1-$ $\gamma \alpha \Delta)=0$, hence, by $(5.21)$,

$$
\operatorname{ord}_{\mathfrak{p}}\left(\Delta_{1}, \ldots, \Delta_{n}\right) \leq \operatorname{ord}_{\mathfrak{p}}(\Delta) .
$$

Together with (5.18) this implies that $\Delta \in\left(\Delta_{1}, \ldots, \Delta_{n}\right)$. Hence (5.22) is solvable in $\xi_{1}, \ldots, \xi_{n} \in O_{S}$. By (5.18) and (5.20) we have

$$
\max \left(h\left(\Delta_{1}\right), \ldots, h\left(\Delta_{n}\right), h(\gamma \Delta)\right) \leq c_{30} .
$$

Now Lemma 11 implies that there are $\xi_{1}, \ldots, \xi_{n}$ satisfying (5.22) with $h\left(\xi_{i}\right) \leq c_{31}$ for $i=1, \ldots, n$. We conclude that $h(C) \leq c_{32}$.

6. Proof of Theorem 2. Let $K$ be an algebraic number field and $S$ a finite set of prime ideals of $O_{K}$. Further, let $(\mathfrak{M}, F)$ be an $O_{S}$-lattice decomposable form pair such that $\operatorname{rank} \mathfrak{M}=n, \operatorname{deg}(F)=r, F$ has splitting field $L$ and $\mathfrak{D}(\mathfrak{M}, F)=\mathfrak{d}$. In what follows, $c_{33}, \ldots, c_{63}$ will denote effectively computable numbers of the form $\left.c|\mathfrak{d}|\right|_{S} ^{c^{\prime}}$, where $c, c^{\prime}$ depend only on $d=$ $[K: \mathbb{Q}],\left|D_{L}\right|, n, r$, the cardinality $s$ of $S$ and the maximum $P$ of the prime numbers lying below the prime ideals in $S$.

Since every $O_{S}$-lattice is isomorphic to a reduced one, we may assume that $\mathfrak{M}$ is reduced. Then, as was seen in Section $2, F$ can be considered as a polynomial in $K\left[X_{1}, \ldots, X_{n}\right]$. We shall prove that there are $\mu \in K^{*}$ and a matrix $U \in G(\mathfrak{M})$ such that the polynomial $F^{\prime}(\mathbf{X})=\mu F(U \mathbf{X})$ has height

$$
h\left(F^{\prime}\right) \leq c_{33} .
$$

This obviously implies Theorem 2 . Since this is trivial for $n=1$, we shall assume $n \geq 2$.

It follows from our assumption that either

$$
\mathfrak{M}=O_{S}^{n} \quad \text { or } \quad \mathfrak{M}=\left(\mathbf{e}_{1}, \ldots, \mathbf{e}_{n-1}, \alpha \mathbf{e}_{n}, \beta \mathbf{e}_{n}\right),
$$

where $\mathfrak{a}=(\alpha, \beta)$ is an integral $O_{S}$-ideal and

$$
h(\alpha) \leq c_{34}, \quad h(\beta) \leq c_{34} .
$$

Let $T$ be the set of prime ideals of $O_{L}$ lying above those in $S$. For $Q(\mathbf{X}) \in$ $L\left[X_{1}, \ldots, X_{n}\right]$, let $(Q)$ be the $O_{T}$-ideal generated by the coefficients of $Q$, and put $|Q|_{T}=N_{L / \mathbb{Q}}(\mathfrak{b})^{1 /[L: \mathbb{Q}]}$, where $\mathfrak{b}$ is the $O_{L}$-ideal composed of prime ideals outside $T$, such that $\mathfrak{b} O_{T}=(Q)$. Further, for $\sigma \in \operatorname{Gal}(L / K)$ we denote by $\sigma(Q)$ the polynomial obtained by applying $\sigma$ to the coefficients of $Q$. We claim that the decomposable form $F$ (considered as a polynomial in $\left.K\left[X_{1}, \ldots, X_{n}\right]\right)$ can be factored as

$$
F(\mathbf{X})=\lambda \prod_{i=1}^{t} l_{i}(\mathbf{X})^{k_{i}}
$$


where $\lambda \in K^{*}, l_{1}, \ldots, l_{t}$ are pairwise non-proportional linear forms in $L\left[X_{1}, \ldots, X_{n}\right]$ and $k_{1}, \ldots, k_{t}$ are positive integers such that

$$
\begin{aligned}
\sigma\left(l_{i}\right)=l_{\sigma(i)}, \quad k_{i}=k_{\sigma(i)} & \text { for } i=1, \ldots, t \\
& \text { and for all } \sigma \in \operatorname{Gal}(L / K),
\end{aligned}
$$

and

$$
l_{i}(\mathbf{X}) \in O_{T}\left[X_{1}, \ldots, X_{n}\right] \quad \text { and } \quad\left|l_{i}\right|_{T} \leq c_{35} \quad \text { for } i=1, \ldots, t,
$$

where $(\sigma(1), \ldots, \sigma(t))$ is a permutation of $(1, \ldots, t)$ for all $\sigma \in \operatorname{Gal}(L / K)$. Namely, it is obvious that there exist $\lambda^{\prime} \in K^{*}$ and linear forms $l_{1}^{\prime}, \ldots, l_{t}^{\prime}$ which satisfy (6.4) and (6.5.a). Then $l_{i}^{\prime}(\mathbf{X}) \in M_{i}\left[X_{1}, \ldots, X_{n}\right]$, where the field $M_{i}$ is defined by

$$
\operatorname{Gal}\left(L / M_{i}\right)=\{\sigma \in \operatorname{Gal}(L / K): \sigma(i)=i\} .
$$

Let $\left(l_{i}^{\prime}\right)$ be the $O_{T}$-ideal generated by the coefficients of $l_{i}^{\prime}$. By Lemma 3(i) and (3.4) there exist $\alpha_{i} \in\left(l_{i}^{\prime}\right)^{-1} \cap M_{i}$ with $\alpha_{i} \neq 0,\left|\alpha_{i}\right|_{T} \leq c_{36}\left|l_{i}^{\prime}\right|_{T}^{-1}$ and $\sigma\left(\alpha_{i}\right)=\alpha_{\sigma(i)}$ for $i=1, \ldots, t$ and $\sigma \in \operatorname{Gal}(L / K)$. Put

$$
\lambda=\lambda^{\prime} \prod_{i=1}^{t} \alpha_{i}^{-k_{i}} \quad \text { and } \quad l_{i}=\alpha_{i} l_{i}^{\prime} \quad \text { for } i=1, \ldots, t .
$$

Then obviously $\lambda \in K^{*}$ and $l_{1}, \ldots, l_{t}$ satisfy (6.5.a) and (6.5.b).

Let $\lambda, l_{1}, \ldots, l_{t}$ satisfy (6.4), (6.5.a), (6.5.b) and let $\mathcal{I}(F)$ be the collection of linearly independent subsets $\left\{l_{i_{1}}, \ldots, l_{i_{n}}\right\}(n=\operatorname{rank} \mathfrak{M})$ of $\left\{l_{1}, \ldots\right.$ $\left.\ldots, l_{t}\right\}$. We denote by $\operatorname{det}\left(l_{i_{1}}, \ldots, l_{i_{n}}\right)$ the coefficient determinant of $l_{i_{1}}, \ldots$ $\ldots, l_{i_{n}}$.

Lemma 13. For each $\left\{l_{i_{1}}, \ldots, l_{i_{n}}\right\} \in \mathcal{I}(F)$, we have

$$
\left|\operatorname{det}\left(l_{i_{1}}, \ldots, l_{i_{n}}\right)\right|_{T} \leq c_{37} .
$$

Proof. By assumption, $\mathfrak{M}$ is one of the $O_{S}$-lattices given in (6.2); we put $\alpha=1, \beta=0, \mathfrak{a}=(1)$ if $\mathfrak{M}=O_{S}^{n}$. For $\mathcal{L}=\left\{l_{i_{1}}, \ldots, l_{i_{n}}\right\} \in \mathcal{I}(F)$, let $\mathfrak{d}(\mathfrak{M}, \mathcal{L})$ be the $O_{T}$-ideal generated by the numbers $\operatorname{det}\left(l_{i_{k}}\left(\mathbf{x}_{j}\right)\right)_{1 \leq k, j \leq n}$ for $\mathbf{x}_{1}, \ldots, \mathbf{x}_{n} \in \mathfrak{M}$. Since $\mathfrak{M} \subseteq O_{S}^{n}$, the ideal $\mathfrak{d}(\mathfrak{M}, \mathcal{L})$ must be divisible by $\operatorname{det}\left(l_{i_{1}}, \ldots, l_{i_{n}}\right)$. Let $\left(l_{i}(\mathfrak{M})\right)$ be the $O_{T}$-ideal generated by the numbers $l_{i}(\mathbf{x})$ with $\mathbf{x} \in \mathfrak{M}$. Then $\left(l_{i}(\mathfrak{M})\right)$ is generated by $l_{i}\left(\mathbf{e}_{1}\right), \ldots, l_{i}\left(\mathbf{e}_{n-1}\right), \alpha l_{i}\left(\mathbf{e}_{n}\right)$ and $\beta l_{i}\left(\mathbf{e}_{n}\right)$, hence it divides $\alpha\left(l_{i}\right)$. Together with (4.6), (6.3) and (6.5.b) this implies that

$$
\left|\left(l_{i}(\mathfrak{M})\right)\right|_{T} \leq|\alpha|_{T} \cdot\left|l_{i}\right|_{T} \leq c_{38} \quad \text { for } i=1, \ldots, t .
$$

By the definition of $\mathfrak{D}(\mathfrak{M}, F)$ we have

$$
\left(\prod_{\mathcal{I}(F)} \operatorname{det}\left(l_{i_{1}}, \ldots, l_{i_{n}}\right)\right)^{2} \supseteq \prod_{\mathcal{I}(F)} \mathfrak{d}(\mathfrak{M}, \mathcal{L})^{2}=\mathfrak{d} \cdot \prod_{\mathcal{I}(F)}\left(l_{i_{1}}(\mathfrak{M}) \ldots l_{i_{n}}(\mathfrak{M})\right)^{2}
$$


hence

$$
\left(\prod_{\mathcal{I}(F)}\left|\operatorname{det}\left(l_{i_{1}}, \ldots, l_{i_{n}}\right)\right|_{T}\right)^{2} \leq c_{39},
$$

where the products are taken over all sets $\mathcal{L}=\left\{l_{i_{1}}, \ldots, l_{i_{n}}\right\}$ in $\mathcal{I}(F)$. Since $l_{i}(\mathbf{X}) \in O_{T}\left[X_{1}, \ldots, X_{n}\right]$, each factor $\left|\operatorname{det}\left(l_{i_{1}}, \ldots, l_{i_{n}}\right)\right|_{T}$ is $\geq 1$. Now Lemma 13 follows at once from (6.6).

We define a hypergraph $\mathcal{G}$ as follows: take as vertices $1, \ldots, t$ and as edges those subsets $I$ of $\{1, \ldots, t\}$ for which $\left\{l_{i}: i \in I\right\}$ is linearly dependent over $L$, while $\left\{l_{i}: i \in I^{\prime}\right\}$ is linearly independent over $L$ for every proper, nonempty subset $I^{\prime}$ of $I$. Thus, for each edge $I$ of $\mathcal{G}$ we have a linear relation

$$
\sum_{i \in I} c_{i}^{(I)} l_{i}=0 \quad \text { identically in } \mathbf{X},
$$

where $c_{i}^{(I)} \in L^{*}$ for $i \in I$. Put $c_{i j}^{(I)}=c_{i}^{(I)} / c_{j}^{(I)}$ for any distinct $i, j \in I$. Then

$$
l_{j}=-\sum_{i \in I \backslash\{j\}} c_{i j}^{(I)} l_{i} \quad \text { for } j \in I .
$$

Since $\left\{l_{i}: i \in I \backslash\{j\}\right\}$ is linearly independent for $j \in I$, this implies that the numbers $c_{i j}^{(I)}$ are uniquely determined by $l_{1}, \ldots, l_{t}$. We claim that

$$
\begin{array}{ll}
m_{T}\left(c_{i j}^{(I)}\right) \leq c_{40} & \text { for each edge } I \text { of } \mathcal{G} \\
& \text { and for any distinct } i, j \in I,
\end{array}
$$

where $m_{T}(\alpha)$ is defined similarly to $m_{S}(\alpha)$ in Section 4 . Indeed, assume for convenience that $I=\{1, \ldots, k\} \cup\{j\}$ with some $j>n$ and that $\left\{l_{1}, \ldots, l_{n}\right\}$ is linearly independent. Then

$$
c_{i j}^{(I)}=-\frac{\operatorname{det}\left(l_{1}, \ldots, l_{i-1}, l_{j}, l_{i+1}, \ldots, l_{n}\right)}{\operatorname{det}\left(l_{1}, \ldots, l_{n}\right)}
$$

and (6.9) follows from Lemma 13. We remark that if the linear forms $l_{i}$ are replaced by $l_{i}^{\prime}=\varepsilon_{i} l_{i}$ for $i=1, \ldots, t$, then by (6.8), the numbers $c_{i j}^{(I)}$ will change into $c_{i j}^{(I)}=\varepsilon_{j} \varepsilon_{i}^{-1} c_{i j}^{(I)}$. The most important part in the proof of Theorem 2 is to show that $\varepsilon_{1}, \ldots, \varepsilon_{t}$ can be chosen so that the linear forms $l_{i}^{\prime}$ still satisfy (6.5.a) and (6.5.b) and that the numbers $c_{i j}^{(I)}$ have small heights. For this, we shall have to use frequently (6.9) and Lemma 8.

In $\mathcal{G}$, a path of length $v$ from $i$ to $j$ with $i, j \in\{1, \ldots, t\}$ is a tuple

$$
C=\left(i_{1}, I_{1}, i_{2}, I_{2}, \ldots, i_{v}, I_{v}, i_{v+1}\right),
$$

where $i_{1}, \ldots, i_{v+1} \in\{1, \ldots, t\}, i_{1}, \ldots, i_{v}$ are pairwise distinct, $i_{1}=i, i_{v+1}=$ $j, i_{u} \neq i_{u+1}$ and $i_{u}, i_{u+1} \in I_{u}$ for some edge $I_{u}$ of $\mathcal{G}$ for $u=1, \ldots, v$. The length of $C$ is denoted by $l(C)$. A shortest path from $i$ to $j$ is a path from 
$i$ to $j$ of minimal length. Put

$$
g(C)=c_{i_{1} i_{2}}^{\left(I_{1}\right)} c_{i_{2} i_{3}}^{\left(I_{2}\right)} \ldots c_{i_{v} i_{v+1}}^{\left(I_{v}\right)} .
$$

We write $C^{-1}=\left(i_{v+1}, I_{v}, i_{v}, \ldots, i_{2}, I_{1}, i_{1}\right)$. If $C_{1}=\left(i_{1}, I_{1}, \ldots, I_{v}, i_{v+1}\right)$ and $C_{2}=\left(i_{v+1}, I_{v+1}, \ldots, I_{m}, i_{m+1}\right)$ then we write $C_{1} C_{2}=\left(i_{1}, I_{1}, \ldots, i_{v+1}\right.$, $\left.I_{v+1}, i_{v+1}, \ldots, I_{m}, i_{m+1}\right)$. Thus

$$
g\left(C^{-1}\right)=g(C)^{-1} \quad \text { and } \quad g\left(C_{1} C_{2}\right)=g\left(C_{1}\right) g\left(C_{2}\right) .
$$

Lemma 14. Let $C_{1}, C_{2}$ be two paths in $\mathcal{G}$ from $i$ to $j$ with $i, j \in\{1, \ldots, t\}$. Then

$$
h\left(g\left(C_{1}\right) / g\left(C_{2}\right)\right) \leq c_{41}^{l\left(C_{1}\right)+l\left(C_{2}\right)} .
$$

Proof. A path $\left(i_{1}, I_{1}, \ldots, I_{v}, i_{1}\right)$ is called a cycle. It is easily seen that, by (6.11), it suffices to show Lemma 14 for paths $C_{1}, C_{2}$ which have no common vertices apart from $i$ and $j$. If $C_{1}, C_{2}$ are two such paths from $i$ to $j$, then $C_{1} C_{2}^{-1}$ is a cycle. So in view of (6.11) it is enough to prove that for every cycle $C$ in $\mathcal{G}$,

$$
h(g(C)) \leq c_{41}^{l(C)} .
$$

Fix a subset $J$ of $\{1, \ldots, t\}$ of cardinality $n$ such that $\left\{l_{j}: j \in J\right\}$ is linearly independent. For each edge $I$ of $\mathcal{G},\left\{l_{i}: i \in I\right\}$ is linearly dependent, hence $\left.{ }^{3}\right)|I \backslash J| \geq 1$. We say that a cycle $C=\left(i_{1}, I_{1}, \ldots, I_{v}, i_{1}\right)$ is $J$-admissible if $\left|I_{1} \backslash J\right|=\ldots=\left|I_{v} \backslash J\right|=1$. We first prove (6.12) for $J$-admissible cycles.

A $J$-admissible cycle $C=\left(i_{1}, I_{1}, \ldots, i_{v}, I_{v}, i_{1}\right)$ is called minimal if either $v=2$ or $v \geq 3$ and there are no $p, q \in\{1, \ldots, v\}$ with $p<q$ and $\{p, q\} \neq$ $\{1,2\},\{2,3\}, \ldots,\{v-1, v\},\{1, v\}$ and an edge $I$ of $\mathcal{G}$ such that $|I \backslash J|=1$ and $i_{p}, i_{q} \in I$. For such a minimal $J$-admissible cycle with $v \geq 3$ we must have $i_{1}, \ldots, i_{v} \in J$. Indeed, suppose that $i_{u} \notin J$ for some $u$ with $1 \leq u \leq v$. Then there is a unique subset $H$ of $J$ such that

$$
l_{i_{u}}=\sum_{h \in H} d_{h} l_{h}
$$

for some $d_{h} \in L^{*}$. Now $I_{u-1}=I_{u}=\left\{i_{u}\right\} \cup H$ (with the convention that $I_{0}:=I_{v}$ ) and so $i_{u-1}$ and $i_{u+1}$ (with $i_{v+1}:=i_{1}$ ) belong to $\left\{i_{u}\right\} \cap H$, which is impossible by the minimality of $C$.

We shall prove that for every $J$-admissible cycle $C$ of length $\geq 3$ there are minimal $J$-admissible cycles $C_{1}, \ldots, C_{w}$ such that $w \leq l(C)-2$ and

$$
g(C)=g\left(C_{1}\right) \ldots g\left(C_{w}\right) .
$$

$\left({ }^{3}\right)$ By $|A|$ we denote the cardinality of a set $A$. 
We proceed by induction on $l(C)$. Every $J$-admissible cycle of length 3 must be minimal, which proves $(6.13)$ for such cycles $C$. Assume that (6.13) holds for all $J$-admissible cycles of length $<v$ where $v \geq 4$, and let $C=\left(i_{1}, I_{1}, \ldots, I_{v}, i_{1}\right)$ be a $J$-admissible cycle. If $C$ is minimal then (6.13) obviously holds with $C_{1}=C$. Hence we assume that $C$ is not minimal. Then there are $p, q \in\{1, \ldots, v\}$ with $p<q$ and $\{p, q\} \neq\{1,2\}, \ldots$ $\ldots,\{v-1, v\},\{1, v\}$ and an edge $I$ of $\mathcal{G}$ with $|I \backslash J|=1$ containing $i_{p}$ and $i_{q}$. Put $C^{\prime}=\left(i_{1}, I_{1}, \ldots, i_{p}, I, i_{q}, \ldots, i_{1}\right), C^{\prime \prime}=\left(i_{p}, I_{p+1}, \ldots, i_{q}, I, i_{p}\right)$. Then $C^{\prime}, C^{\prime \prime}$ are $J$-admissible cycles with $3 \leq l\left(C^{\prime}\right)<l(C), 3 \leq l\left(C^{\prime \prime}\right)<l(C)$ and $l\left(C^{\prime}\right)+l\left(C^{\prime \prime}\right)=l(C)+2$. Further, $g(C)=g\left(C^{\prime}\right) g\left(C^{\prime \prime}\right)$. Now (6.13) follows for $C$, by applying the induction hypothesis to $C^{\prime}$ and $C^{\prime \prime}$.

In view of $(6.13),(6.12)$ follows for $J$-admissible cycles, once we have proved that for every minimal $J$-admissible cycle $C$,

$$
h(g(C)) \leq c_{42} \text {. }
$$

The only minimal $J$-admissible cycles of length 2 are of the form $\left(i_{1}, I, i_{2}\right.$, $\left.I, i_{1}\right)$ and for such cycles $C$ one has $g(C)=1$. So we only consider minimal $J$-admissible cycles of length $\geq 3$. As we showed above, all vertices of such a minimal cycle $C$ belong to $J$. For convenience we assume that $J=\{1, \ldots, n\}$ and that $C=\left(1, I_{1}, 2, I_{2}, \ldots, v, I_{v}, 1\right)$ is a minimal $J$-admissible cycle with $v \geq 3$. Let $p_{u}$ be the element of $I_{u}$ not belonging to $J$, and let $I_{u}^{\prime}=I_{u} \cap\{v+1, \ldots, n\}$ for $u=1, \ldots, v$. Then, by (6.8),

$$
l_{p_{u}}=d_{u u} l_{u}+d_{u, u+1} l_{u+1}+\sum_{j \in I_{u}^{\prime}} d_{u j} l_{j} \quad \text { for } u=1, \ldots, v-1,
$$

and

$$
l_{p_{v}}=d_{v 1} l_{1}+d_{v v} l_{v}+\sum_{j \in I_{v}^{\prime}} d_{v j} l_{j}
$$

where $d_{u j}=-c_{j, p_{u}}^{\left(I_{u}\right)}$ for $u=1, \ldots, v$. This implies that

$$
\begin{aligned}
& \frac{\operatorname{det}\left(l_{p_{1}}, \ldots, l_{p_{v}}, l_{v+1}, \ldots, l_{n}\right)}{\operatorname{det}\left(l_{1}, \ldots, l_{n}\right)} \\
& =\left|\begin{array}{ccccc}
d_{11} & d_{12} & & & 0 \\
& d_{22} & d_{23} & & \\
\vdots & & \ddots & \ddots & \\
& 0 & & d_{v-1, v-1} & d_{v-1, v} \\
d_{1 v} & & & & d_{v v}
\end{array}\right| \\
& =d_{11} d_{22} \ldots d_{v v} \pm d_{12} \ldots d_{v-1, v} d_{1 v} .
\end{aligned}
$$

Put $\xi=d_{11} \ldots d_{v v}, \eta=d_{12} \ldots d_{v-1, v} d_{1 v}$, and denote the left-hand side of (6.15) by $\zeta$. Then, by (6.15), $\xi \pm \eta=\zeta$. Since $d_{u j} / d_{u k}=c_{j k}^{\left(I_{u}\right)}$ for $j, k \in I_{u}$, 
we have

$$
\xi / \eta= \pm g(C) .
$$

Further, by $(6.9)$ and $(4.2), m_{T}(\xi) \leq c_{43}, m_{T}(\eta) \leq c_{43}$ and by Lemma 13 we also have $m_{T}(\zeta) \leq c_{43}$. If $\zeta=0$, then $\xi / \eta= \pm 1$. If $\zeta \neq 0$ then by Lemma $8, h(\xi / \eta) \leq c_{44}$. Now (6.16) implies that $h(g(C)) \leq c_{42}$. This proves (6.14).

For every path $C=\left(i_{1}, I_{1}, \ldots, i_{v}, I_{v}, i_{v+1}\right)$ in $\mathcal{G}$ we put

$$
m(C)=\max \left\{\left|I_{u} \backslash J\right|: 1 \leq u \leq v\right\} .
$$

Obviously, $m(C) \geq 1$. We shall prove that for every cycle $C$ in $\mathcal{G}$ with $m(C)=m \geq 1$, there is a $J$-admissible cycle $C^{\prime}$ in $\mathcal{G}$ with $l\left(C^{\prime}\right) \leq 2^{m-1} l(C)$ and

$$
h\left(g(C) / g\left(C^{\prime}\right)\right) \leq c_{45}^{l(C)} .
$$

Since $m \leq n$, this implies Lemma 14 .

We shall prove (6.17) by induction on $m(C)$. For $m(C)=1$ we are done. Let $m \geq 2$ and assume that (6.17) holds for all cycles $C$ in $\mathcal{G}$ with $m(C)<m$. Let $C=\left(i_{1}, I_{1}, \ldots, i_{v}, I_{v}, i_{1}\right)$ be a cycle with $m(C)=m$ and put $i_{v+1}:=i_{1}$. In view of (6.11), it suffices to prove that for each $u \in\{1, \ldots, v\}$ with $\left|I_{u} \backslash J\right|=m$, there is a path $C_{u}$ from $i_{u}$ to $i_{u+1}$ such that

$$
h\left(\frac{g\left(i_{u}, I_{u}, i_{u+1}\right)}{g\left(C_{u}\right)}\right) \leq c_{46}, \quad l\left(C_{u}\right) \leq 2, \quad m\left(C_{u}\right) \leq m-1 .
$$

For convenience, we write $i_{u}=i, i_{u+1}=j, I_{u}=I$. First assume that there are an edge $I^{\prime}$ of $\mathcal{G}$ and a subset $J^{\prime}$ of $\{1, \ldots, t\}$ of cardinality $n$ such that $\left\{l_{j}: j \in J^{\prime}\right\}$ is linearly independent, and

$$
i, j \in I^{\prime}, \quad\left|I \backslash J^{\prime}\right|=1, \quad\left|I^{\prime} \backslash J^{\prime}\right|=1, \quad\left|I^{\prime} \backslash J\right| \leq m-1 .
$$

Then the cycle $C_{0}=\left(i, I, j, I^{\prime}, i\right)$ is $J^{\prime}$-admissible and, by (6.11) and (6.12),

$$
h\left(g(i, I, j) / g\left(i, I^{\prime}, j\right)\right)=h\left(g\left(C_{0}\right)\right) \leq c_{47},
$$

which proves (6.18). Now assume that there are no sets $I^{\prime}, J^{\prime}$ with the properties specified above. Choose $p$ from $I$ with $p \notin J$. Let $H$ be a subset of $J$ of cardinality $n-|I|+1$ such that if $G:=(I \backslash\{p\}) \cup H$, then $\left\{l_{k}: k \in G\right\}$ is linearly independent and has cardinality $n$. Then by (6.8)

$$
l_{p}=\sum_{k \in G} d_{k} l_{k} \quad \text { with } d_{k}= \begin{cases}-c_{k p}^{(I)} & \text { for } k \in I \backslash\{p\}, \\ d_{k}=0 & \text { for } k \notin I \backslash\{p\} .\end{cases}
$$

Since $|G \cap J|=|(I \backslash\{p\}) \backslash J|=m-1 \geq 1$, there is a $q \in J$ with $q \notin G$. We can express $l_{q}$ uniquely as

$$
l_{q}=\sum_{k \in G} e_{k} l_{k} \quad \text { with } e_{k} \in L .
$$


There is a $z \in I \backslash\{p\}$ with $e_{z} \neq 0$ since $\left\{l_{k}: k \in J\right\}$ is linearly independent. Since $z \in I \backslash\{p\}$ we also have $d_{z} \neq 0$. From (6.19) it follows that

$$
l_{z}=d_{z}^{-1} l_{p}-\sum_{k \in G \backslash\{z\}}\left(d_{k} / d_{z}\right) l_{k} .
$$

By substituting this into (6.20) we get

$$
\begin{aligned}
& l_{q}=\sum_{k \in G^{\prime}} f_{k} l_{k} \quad \text { with } G^{\prime}=G \cup\{p\} \backslash\{z\}, \\
& f_{p}=e_{z} / d_{z}, \quad f_{k}=e_{k}-f_{p} d_{k} \quad \text { for } k \neq p .
\end{aligned}
$$

Note that $\left|G^{\prime}\right|=n$ and that $\left\{l_{k}: k \in G^{\prime}\right\}$ is linearly independent. The sets $I^{\prime}=\{q\} \cup\left\{k \in G: e_{k} \neq 0\right\}$ and $I^{\prime \prime}=\{q\} \cup\left\{k \in G^{\prime}: f_{k} \neq 0\right\}$ are edges of $\mathcal{G}$. Further,

$$
\begin{aligned}
& |I \backslash G|=1, \quad\left|I^{\prime} \backslash G\right|=1, \quad\left|I^{\prime} \backslash J\right| \leq m-1, \\
& \left|I \backslash G^{\prime}\right|=1, \quad\left|I^{\prime \prime} \backslash G^{\prime}\right|=1, \quad\left|I^{\prime \prime} \backslash J\right| \leq m-1 .
\end{aligned}
$$

By our assumption, neither $I^{\prime}$ nor $I^{\prime \prime}$ contains both $i$ and $j$. Assume for instance that $j \notin I^{\prime}$. Then either $j \neq p$, in which case we have $e_{j}=0$, so $j \neq z$, whence $f_{j}=-f_{p} d_{j} \neq 0$, that is $j \in I^{\prime \prime}$; or $j=p$, in which case $f_{j}=f_{p} \neq 0$ and also $j \in I^{\prime \prime}$. Therefore, $i \notin I^{\prime \prime}$ and $0=f_{i}=e_{i}-f_{p} d_{i}$. But then $e_{i} \neq 0$ and $i \in I^{\prime}$. Let $C_{u}=\left(i, I^{\prime}, q, I^{\prime \prime}, j\right)$. Then in view of $e_{i}=f_{p} d_{i}$, $f_{j}=-f_{p} d_{j}\left(\right.$ with $\left.d_{p}:=-1\right)$ we have

$$
g\left(C_{u}\right)=c_{i q}^{\left(I^{\prime}\right)} c_{q j}^{\left(I^{\prime \prime}\right)}=e_{i} f_{j}^{-1}=-\frac{f_{p} d_{i}}{f_{p} d_{j}}=-\frac{d_{i}}{d_{j}}=-g(i, I, j) .
$$

This implies (6.18). The proof of Lemma 14 is now complete.

LEMma 15. There are $\varepsilon_{1}, \ldots, \varepsilon_{t} \in O_{T}^{*}$ such that

$$
\begin{aligned}
& \sigma\left(\varepsilon_{i}\right)=\varepsilon_{\sigma(i)} \quad \text { for } i=1, \ldots, t \text { and for each } \sigma \in \operatorname{Gal}(L / K) \\
& h\left(\frac{\varepsilon_{j}}{\varepsilon_{i}} c_{i j}^{(I)}\right) \leq c_{48}
\end{aligned}
$$

for every edge $I$ of $\mathcal{G}$ and for all distinct $i, j \in I$.

Proof. We apply Lemma 7. For distinct $i, j \in\{1, \ldots, t\}$, let $P(i, j)$ be the collection of shortest paths in $\mathcal{G}$ from $i$ to $j$ and set $P(i, j)=\emptyset$ if no path between $i$ and $j$ exists. For a non-isolated vertex $i$ of $\mathcal{G}$, let $A_{i}$ be the set consisting of all numbers of the form

$$
\prod_{j: P(i, j) \neq \emptyset} g\left(C_{i j}\right),
$$

where $C_{i j}$ is any path in $P(i, j)$, and for an isolated vertex $i$ in $\mathcal{G}$ let $A_{i}=$ $\{1\}$. By (6.5.a), each $\sigma \in \operatorname{Gal}(L / K)$ maps linearly (in)dependent subsets 
of $\left\{l_{1}, \ldots, l_{t}\right\}$ onto linearly (in)dependent subsets, hence it maps edges of $\mathcal{G}$ onto edges of $\mathcal{G}$, and

$$
\sigma\left(c_{i j}^{(I)}\right)=c_{\sigma(i), \sigma(j)}^{\sigma(I)}
$$

for each edge $I$ of $\mathcal{G}$ and each distinct $i, j \in I$. This implies that

$$
\sigma\left(A_{i}\right)=A_{\sigma(i)} \quad \text { for } i=1, \ldots, t \text { and for each } \sigma \in \operatorname{Gal}(L / K) .
$$

Further, if $i$ is not isolated then each element $\alpha$ of $A_{i}$ is the product of numbers of the form $c_{p q}^{(I)}$, and each shortest path between two vertices has length at most $r$. Hence we have, by (6.9) and (4.2),

$$
m_{T}(\alpha) \leq c_{49} \quad \text { for each } \alpha \in A_{i} \text { and } i=1, \ldots, t .
$$

Further, by Lemma 14 we have

$$
h(\alpha / \beta) \leq c_{50} \quad \text { for each } \alpha, \beta \in A_{i} \text { and } i=1, \ldots, t .
$$

Each $\sigma \in \operatorname{Gal}(L / K)$ maps connected components of $\mathcal{G}$ onto connected components. Let $\mathcal{C}$ be a $\operatorname{Gal}(L / K)$-orbit of $\{1, \ldots, t\}$ as defined in Section 3 . The connected components of $\mathcal{G}$ containing an element of $\mathcal{C}$ as vertex have the same cardinality which will be denoted by $n_{\mathcal{C}}$. By Lemma $7,(6.24)$, (6.25) and (6.26), there are $\varepsilon_{1}, \ldots, \varepsilon_{t} \in O_{T}^{*}$ satisfying (6.22) and

$$
h\left(\varepsilon_{i}^{-n_{\mathcal{C}}} \alpha\right) \leq c_{51} \quad \text { for each } \alpha \in A_{i}, i \in \mathcal{C} .
$$

We now prove that these $\varepsilon_{1}, \ldots, \varepsilon_{t}$ satisfy (6.23). Let $I$ be an edge of $\mathcal{G}$ and $i, j \in I$. Suppose that $i \in \mathcal{C}, j \in \mathcal{C}^{\prime}$ for $\operatorname{Gal}(L / K)$-orbits $\mathcal{C}, \mathcal{C}^{\prime}$. Then $n_{\mathcal{C}}$ and $n_{\mathcal{C}^{\prime}}$ have the same value, say $n^{\prime}$. It is clear that $n^{\prime} \leq t \leq r$. Take

$$
\alpha_{i}=\prod_{k: P(i, k) \neq \emptyset} g\left(C_{i k}\right) \quad \text { and } \quad \alpha_{j}=\prod_{k: P(j, k) \neq \emptyset} g\left(C_{j k}^{\prime}\right),
$$

where $C_{i k}$ is any shortest path from $i$ to $k$ and $C_{j k}^{\prime}$ any shortest path from $j$ to $k$. By Lemma 14 and (6.11) we have $k \neq i, j$,

$$
\begin{gathered}
h\left(c_{i j}^{(I)} / g\left(C_{i j}\right)\right) \leq c_{52}, \\
h\left(c_{i j}^{(I)} \cdot g\left(C_{j i}^{\prime}\right)\right) \leq c_{52}, \quad h\left(c_{i j}^{(I)} \cdot g\left(C_{j k}^{\prime} C_{i k}^{-1}\right)\right) \leq c_{52},
\end{gathered}
$$

hence

$$
h\left(\left(c_{i j}^{(I)}\right)^{q} \cdot \alpha_{j} / \alpha_{i}\right) \leq c_{53}, \quad \text { where } q=n^{\prime}-1 .
$$

Together with (6.27) this yields

$$
h\left(\frac{\varepsilon_{j}}{\varepsilon_{i}} c_{i j}^{(I)}\right) \leq\left[h\left(\frac{\varepsilon_{i}^{-q} \alpha_{i}}{\varepsilon_{j}^{-q} \alpha_{j}}\right) \cdot h\left(\left(c_{i j}^{(I)}\right)^{q} \frac{\alpha_{j}}{\alpha_{i}}\right)\right]^{1 / q} \leq c_{48} .
$$

This proves Lemma 15 .

In what follows, we put $l_{i}^{\prime}=\varepsilon_{i} l_{i}$ for $i=1, \ldots, t$, and $c_{i j}^{\prime(I)}=\left(\varepsilon_{j} / \varepsilon_{i}\right) c_{i j}^{(I)}$. Note that $l_{1}^{\prime}, \ldots, l_{t}^{\prime}$ satisfy (6.5.a) and (6.5.b). 
Lemma 16. There are a linearly independent set of linear forms $\left\{m_{1}, \ldots\right.$ $\left.\ldots, m_{n}\right\}$ with $m_{i} \in O_{S}\left[X_{1}, \ldots, X_{n}\right]$ and numbers $d_{i j} \in L$ such that

$$
\begin{gathered}
l_{i}^{\prime}=\sum_{j=1}^{n} d_{i j} m_{j} \quad \text { for } i=1, \ldots, t, \\
h\left(d_{i j}\right) \leq c_{54} \quad \text { for } i=1, \ldots, t \text { and } j=1, \ldots, n .
\end{gathered}
$$

Proof. Partition $\{1, \ldots, t\}$ into $\operatorname{Gal}(L / K)$-orbits as in Section 3. Assume for the moment that $\{1, \ldots, u\}$ is such an orbit. Define $M_{1}$ by $\operatorname{Gal}\left(L / M_{1}\right)=\{\sigma \in \operatorname{Gal}(L / K): \sigma(1)=1\}$. By [16], Satz 6, $M_{1}$ has a $\mathbb{Q}$-basis $\left\{\omega_{1}, \ldots, \omega_{m}\right\}$ with

$$
\begin{aligned}
\left|\sigma\left(\omega_{i}\right)\right| \leq c_{55}, \quad \omega_{i} \in O_{M_{1}} \\
\\
\text { for } i=1, \ldots, m \text { and for each } \sigma \in \operatorname{Gal}(L / K) .
\end{aligned}
$$

We may assume that $\left\{\omega_{1}, \ldots, \omega_{u}\right\}$ is a $K$-basis of $M_{1}$. Then there are linear forms $n_{1}, \ldots, n_{u} \in K\left[X_{1}, \ldots, X_{n}\right]$ such that

$$
l_{1}^{\prime}=\sum_{j=1}^{u} \omega_{j} n_{j} .
$$

Pick $\sigma_{i} \in \operatorname{Gal}(L / K)$ such that $\sigma_{i}(1)=i$ for $i=1, \ldots, u$ and put

$$
d:=\left(\operatorname{det}\left(\sigma_{i}\left(\omega_{j}\right)\right)_{1 \leq i, j \leq u}\right)^{2} .
$$

Then $d \in K^{*}$ and the linear forms $m_{j}=d n_{j}(j=1, \ldots, u)$ have their coefficients in $O_{S}$. Put $\omega_{i j}=d^{-1} \sigma_{i}\left(\omega_{j}\right)$ for $i, j \in\{1, \ldots, u\}$. Then, by (6.28)

$$
\begin{gathered}
l_{i}^{\prime}=\sum_{j=1}^{u} \omega_{i j} m_{j} \quad \text { for } i=1, \ldots, u, \\
h\left(\omega_{i j}\right) \leq c_{56} \quad \text { for } 1 \leq i, j \leq u .
\end{gathered}
$$

By applying this argument to the other $\operatorname{Gal}(L / K)$-orbits we find linear forms $m_{1}, \ldots, m_{t} \in O_{S}\left[X_{1}, \ldots, X_{n}\right]$ and an invertible matrix $\Omega=\left(\omega_{i j}\right)$ with entries in $L$ such that

$$
l_{i}^{\prime}=\sum_{j=1}^{t} \omega_{i j} m_{j} \quad \text { for } i=1, \ldots, t \quad \text { and } \quad h(\Omega) \leq c_{57} .
$$

We assume that $\left\{l_{1}^{\prime}, \ldots, l_{n}^{\prime}\right\}$ and $\left\{m_{1}, \ldots, m_{n}\right\}$ are linearly independent, which is no restriction. Every linear form $l_{i}^{\prime}(i=n+1, \ldots, t)$ can be expressed uniquely as

$$
l_{i}^{\prime}=\sum_{j=1}^{n} e_{i j} l_{j}^{\prime},
$$


where $E:=\left(e_{i j}\right) \in L^{t-n, n}$. The sets $I_{i}=\{i\} \cup\left\{j: e_{i j} \neq 0\right\}$ are edges of $\mathcal{G}$, hence, in (6.31), either $e_{i j}=0$ or $e_{i j}=-c_{j i}^{\left(I_{i}\right)}$ by (6.8). Now Lemma 15 implies that

$$
h(E) \leq c_{58} .
$$

Since $\left\{m_{1}, \ldots, m_{n}\right\}$ is linearly independent there is a matrix $D=\left(d_{i j}\right) \in$ $L^{t, n}$ such that

$$
l_{i}^{\prime}=\sum_{j=1}^{n} d_{i j} m_{j} \quad \text { for } i=1, \ldots, t .
$$

We can express the entries of $D$ as rational functions in the entries of $\Omega$ and $E$ : first, by expressing $m_{1}, \ldots, m_{t}$ as linear combinations of $l_{1}^{\prime}, \ldots, l_{t}^{\prime}$, which is possible since $\Omega$ is invertible; secondly, by expressing $m_{1}, \ldots, m_{n}$ as linear combinations of $l_{1}^{\prime}, \ldots, l_{n}^{\prime}$, which can be done by (6.31); thirdly, by expressing $l_{1}^{\prime}, \ldots, l_{n}^{\prime}$ as linear combinations of $m_{1}, \ldots, m_{n}$; and finally, by expressing $l_{1}^{\prime}, \ldots, l_{t}^{\prime}$ as linear combinations of $m_{1}, \ldots, m_{n}$, using (6.31). Hence it follows that $h(D) \leq c_{54}$.

Proof of Theorem 2. Let $d_{i j}$ be numbers and $m_{1}, \ldots, m_{n}$ linear forms with the properties specified in the statement of Lemma 16. Let $B \in O_{S}^{n, n}$ be the matrix whose $i$ th row consists of the coefficients of $m_{i}$ and put

$$
G(\mathbf{X})=\prod_{i=1}^{t}\left(\sum_{j=1}^{n} d_{i j} X_{j}\right),
$$

where $\mathbf{X}=\left(X_{1}, \ldots, X_{n}\right)^{T}$. From Lemma 16 and the construction of $l_{1}^{\prime}, \ldots$ $\ldots, l_{t}^{\prime}$, it follows that there is a $\mu \in K^{*}$ with

$$
\mu F(\mathbf{X})=G(B \mathbf{X}) .
$$

Assume that $\left\{l_{1}^{\prime}, \ldots, l_{n}^{\prime}\right\}$ is linearly independent and let $A \in L^{n, n}$ be the matrix whose $i$ th row consists of the coefficients of $l_{i}^{\prime}$. By Lemma 13 we have $|\operatorname{det} A|_{T}=\left|\operatorname{det}\left(l_{1}, \ldots, l_{n}\right)\right|_{T} \leq c_{37}$. Further, by Lemma 16, there is an invertible matrix $D \in L^{n, n}$ with $A=D B$ and $h(D) \leq c_{59}$. Then, by (4.6), $\left|\operatorname{det} D^{-1}\right|_{T} \leq h\left(\operatorname{det} D^{-1}\right)=h(\operatorname{det} D) \leq c_{60}$. Hence

$$
|\operatorname{det} B|_{S} \leq c_{61} \text {. }
$$

By our assumption, the $O_{S^{-}}$lattice $\mathfrak{M}$ is equal to either $O_{S}^{n}$ or $\left(\mathbf{e}_{1}, \ldots\right.$ $\left.\ldots, \mathbf{e}_{n-1}, \alpha \mathbf{e}_{n}, \beta \mathbf{e}_{n}\right)$ with $h(\alpha) \leq c_{34}$ and $h(\beta) \leq c_{34}$. Put $\mathfrak{a}=(1)$ if $\mathfrak{M}=O_{S}^{n}$ and $\mathfrak{a}=(\alpha, \beta)$ otherwise. In the second case we have, by (4.6),

$$
|\mathfrak{a}|_{S} \leq \max \left(|\alpha|_{S},|\beta|_{S}\right) \leq \max (h(\alpha), h(\beta)) \leq c_{62} ;
$$

in the first case, this evidently holds. By Lemma 9, there is a matrix $U \in$ 
$G(n, \mathfrak{a})$ such that the matrix $B^{\prime}=B U$ satisfies

$$
h\left(B^{\prime}\right) \leq c_{63} .
$$

Note that by (5.1), the matrix $U$ belongs to $G(\mathfrak{M})$. Put

$$
F^{\prime}(\mathbf{X})=G\left(B^{\prime} \mathbf{X}\right)
$$

then, by (6.33), $\mu F(U \mathbf{X})=F^{\prime}(\mathbf{X})$. Finally, by (6.32), Lemma 16 and (6.34), we have $h\left(F^{\prime}\right) \leq c_{33}$. This proves $(6.1)$ and hence Theorem 2 .

7. Proof of Corollaries. As above, $K$ is an algebraic number field of degree $d$ and $S$ is a finite set of prime ideals of $O_{K}$ of cardinality $s$ such that the largest of the prime numbers lying below prime ideals of $S$ is equal to $P$. Let $(\mathfrak{M}, F)$ be an $O_{S}$-lattice decomposable form pair such that rank $\mathfrak{M}=n$, $\operatorname{deg}(F)=r, F$ has splitting field $L$ and $D(\mathfrak{M}, F)=\mathfrak{d}$.

Proof of Corollary 1 . By Theorem $2,(\mathfrak{M}, F)$ is weakly equivalent to a pair $\left(\mathfrak{M}^{\prime}, F^{\prime}\right)$, where $\mathfrak{M}^{\prime}$ is a reduced $O_{S}$-lattice, and $h\left(F^{\prime}\right) \leq c_{64}$ with an effectively computable number $c_{64}$ depending only on $d,\left|D_{L}\right|, s, P, n, r$ and $|\mathfrak{d}|_{S}$. Hence it suffices to prove the following lemma.

Lemma 17. We have $\left|D_{L}\right| \leq c_{65}$, where $c_{65}$ is an effectively computable number depending only on $d,\left|D_{K}\right|, s, P, n, r$ and $|\mathfrak{d}|_{S}$.

Proof. $c_{66}$ and $c_{67}$ will denote effectively computable numbers depending only on the parameters listed in Lemma 17 . $F$ can be factored as in (3.1), into linear functions $l_{1}, \ldots, l_{t}$ satisfying (3.2). Define the fields $M_{i}$ $(i=1, \ldots, t)$ as in $(3.3)$. Let $\mathcal{C}_{1}, \ldots, \mathcal{C}_{u}$ be the $\operatorname{Gal}(L / K)$-orbits of $\{1, \ldots, t\}$ relative to the $\operatorname{Gal}(L / K)$-action defined in (3.2) and assume that $i \in \mathcal{C}_{i}$ for $i=1, \ldots, u$. Let $O_{S, i}$ be the integral closure of $O_{S}$ in $M_{i}$, and $\mathfrak{d}_{i}$ the discriminant of the ring extension $O_{S, i} / O_{S}$. Then, by Lemma 2 ,

$$
\left|\mathfrak{d}_{1} \ldots \mathfrak{d}_{u}\right|_{S} \leq|\mathfrak{d}|_{S}^{n / 2} \leq c_{66}
$$

By Lemma 14 of [6], we have

$$
\left|D_{M_{i}}\right| \leq c_{67}|\mathfrak{d}|_{S} \quad \text { for } i=1, \ldots, u .
$$

Let $m_{i}=\left[M_{i}: K\right]$ for $i=1, \ldots, u$. Since $L$ is the composite of $M_{1}, \ldots, M_{t}$ we have, by a result of Stark ([19], Lemma 7),

$$
D_{L} \mid \prod_{i=1}^{t} D_{M_{i}}^{\left[L: M_{i}\right]}=\prod_{i=1}^{u} D_{M_{i}}^{m_{i}\left[L: M_{i}\right]}=\left(\prod_{i=1}^{u} D_{M_{i}}\right)^{[L: K]} .
$$

Together with $(7.1),(7.2)$ and the inequality $[L: K] \leq r$ !, this proves Lemma 17.

Pro of of Corollary 2. We shall frequently use the following facts: (i) for every $C \geq 1$ it is possible to determine effectively a finite set con- 
taining all $\alpha \in K$ with $h(\alpha) \leq C$ (see e.g. [7]), and (ii) for every $O_{S}$-ideal $\mathfrak{a}$ given by a set of generators and every $\alpha \in K$, it is possible to decide whether $\alpha \in \mathfrak{a}$ or not. We remark that by Lemma 11 , it can be decided effectively whether a system of linear equations $A \mathbf{x}=\mathbf{b}$ is solvable in $\mathbf{x} \in O_{S}^{n}$, and fact (ii) is a special case of this.

By Corollary 1 , for given $n, r$ and $\mathfrak{d}$, each weak equivalence class contains an $O_{S}$-lattice decomposable form pair $(\mathfrak{M}, F)$ such that $\mathfrak{M}$ is reduced, $\operatorname{rank} \mathfrak{M}=n, \operatorname{deg}(F)=r, \mathfrak{D}(\mathfrak{M}, F)=\mathfrak{d}$ and $h(F) \leq c_{68}$, where $c_{68}$ is an effectively computable number depending only on $d, s, P, n, r,\left|D_{K}\right|$ and $|\mathfrak{d}|_{S}$. It is possible to determine effectively a finite set containing all pairs $(\mathfrak{M}, F)$ with these properties; what remains is to find an effective method to decide whether any two pairs $\left(\mathfrak{M}_{1}, F_{1}\right),\left(\mathfrak{M}_{2}, F_{2}\right)$ in that finite set are weakly equivalent or not.

First we give a procedure to determine effectively whether any two reduced lattices are isomorphic. This is trivial if $\mathfrak{M}_{1}$ or $\mathfrak{M}_{2}$ is $O_{S}^{n}$. Hence we may assume that $\mathfrak{M}_{1}=\left(\mathbf{e}_{1}, \ldots, \mathbf{e}_{n-1}, \alpha \mathbf{e}_{n}, \beta \mathbf{e}_{n}\right), \mathfrak{M}_{2}=\left(\mathbf{e}_{1}, \ldots, \mathbf{e}_{n-1}, \gamma \mathbf{e}_{n}\right.$, $\left.\delta \mathbf{e}_{n}\right)$, where $\alpha, \beta, \gamma, \delta$ are given elements of $O_{S}$ with $h(\alpha), h(\beta), h(\gamma), h(\delta) \leq$ $c_{69}$ for some effectively computable number $c_{69}$ depending only on $d$ and $\left|D_{K}\right|$. If there is an $a \in K^{*}$ with $a(\alpha, \beta)=(\gamma, \delta)$ then, by (4.6), $m_{S}(a) \leq c_{70}$ where $c_{70}$ is also an effectively computable number depending only on $d$ and $\left|D_{K}\right|$. By Lemma 6 , there is an $\varepsilon \in O_{S}^{*}$ with $h(\varepsilon a) \leq c_{71}$, where $c_{71}$ is an effectively computable number depending only on $d,\left|D_{K}\right|, S$ and $P$. This implies that there is a $b \in K^{*}$ with $(b \alpha, b \beta)=(\gamma, \delta)$ and $h(b) \leq c_{71}$. Hence in order to decide whether $(\alpha, \beta),(\gamma, \delta)$ belong to the same ideal class it suffices to check, for each $b$ in some effectively computable finite set, whether the $O_{S}$-ideals $(b \alpha, b \beta)$ and $(\gamma, \delta)$ are equal.

So we can restrict ourselves to pairs $(\mathfrak{M}, F)$ where $\mathfrak{M}$ is a fixed, given reduced $O_{S}$-lattice, and hence a reduced $O_{S}$-sublattice of $O_{S}^{n}$ of rank $n$. Two $O_{S^{-}}$-lattice decomposable form pairs $\left(\mathfrak{M}, F_{1}\right)$ and $\left(\mathfrak{M}, F_{2}\right)$ are weakly equivalent if and only if there are $\lambda \in K^{*}$ and a matrix $U \in G(\mathfrak{M})$ such that $F_{2}(\mathbf{X})=\lambda F_{1}(U \mathbf{X})$ (cf. Section 1). For a given matrix $U \in K^{n, n}$ it can be decided whether $U \in G(\mathfrak{M})$ and $F_{2}(\mathbf{X})=\lambda F_{1}(U \mathbf{X})$ for some $\lambda \in K^{*}$. Therefore, it suffices to prove the following lemma.

Lemma 18. Let $\mathfrak{M}$ be a reduced $O_{S}$-lattice, and let $F_{1}(\mathbf{X}), F_{2}(\mathbf{X})$ be two decomposable forms on $K \mathfrak{M}$ of degree $r$ and maximal rank $n$ such that

$$
F_{2}(\mathbf{X})=\lambda F_{1}(U \mathbf{X})
$$

for some $\lambda \in K^{*}$ and $U \in G(\mathfrak{M})$. Then there are $\lambda^{\prime} \in K^{*}$ and $U^{\prime} \in G(\mathfrak{M})$ such that

$$
F_{2}(\mathbf{X})=\lambda^{\prime} F_{1}\left(U^{\prime} \mathbf{X}\right) \quad \text { and } \quad h\left(U^{\prime}\right) \leq c_{72}
$$


where $c_{72}$ is an effectively computable number depending only on $d,\left|D_{K}\right|, s$, $P, n, r, h\left(F_{1}\right)$ and $h\left(F_{2}\right)$.

P r o o f. $c_{73}, \ldots, c_{81}$ will denote effectively computable numbers depending only on the parameters specified in Lemma 18. By (7.3), we can express $F_{1}, F_{2}$ as

$$
F_{1}(\mathbf{X})=\mu \prod_{i=1}^{t} l_{i}(\mathbf{X})^{k_{i}}, \quad F_{2}(\mathbf{X})=\nu \prod_{i=1}^{t} m_{i}(\mathbf{X})^{k_{i}},
$$

where $\mu, \nu \in K^{*}, k_{1}, \ldots, k_{t}$ are positive integers, $l_{1}, \ldots, l_{t}$ are pairwise nonproportional linear forms and $m_{1}, \ldots, m_{t}$ are pairwise non-proportional linear forms with coefficients in the common splitting field $L$ of $F_{1}$ and $F_{2}$. Let $O_{T}$ be the integral closure of $O_{S}$ in $L$. We claim that $l_{1}, \ldots, l_{t}, m_{1}, \ldots, m_{t}$ can be chosen such that

$$
\begin{gathered}
\text { (7.5.a) } \\
l_{i}(\mathbf{X}) \in O_{T}\left[X_{1}, \ldots, X_{n}\right], \quad m_{i}(\mathbf{X}) \in O_{T}\left[X_{1}, \ldots, X_{n}\right], \\
\text { (7.5.b) } \quad \lambda_{i} m_{i}(\mathbf{X})=l_{i}(U \mathbf{X}) \quad(i=1, \ldots, t) \text { for some } \lambda_{1}, \ldots, \lambda_{t} \in L^{*} ; \\
\text { (7.5.c) } \quad \sigma\left(l_{i}\right)=l_{\sigma(i)}, \quad \sigma\left(m_{i}\right)=m_{\sigma(i)}, \quad k_{\sigma(i)}=k_{i} \\
\\
\text { for } i=1, \ldots, t, \sigma \in \operatorname{Gal}(L / K),
\end{gathered}
$$

where $(\sigma(1), \ldots, \sigma(t))$ is a permutation of $(1, \ldots, t)$ for each $\sigma \in \operatorname{Gal}(L / K)$. Namely, choose linear forms $l_{1}^{\prime}, \ldots, l_{t}^{\prime}, m_{1}^{\prime}, \ldots, m_{t}^{\prime}$ satisfying (7.4), (7.5.b), (7.5.c) such that at least one of the coefficients of each of these forms is equal to 1 . Construct polynomials $f_{1}(X), f_{2}(X)$ from $F_{1}(\mathbf{X})$ and $F_{2}(\mathbf{X})$, respectively, by setting $X_{1}=X, X_{2}=X^{r+1}, \ldots, X_{n}=X^{(r+1)^{n-1}}$, where $r=\operatorname{deg}\left(F_{1}\right)=\operatorname{deg}\left(F_{2}\right)$. Then $h\left(f_{1}\right)=h\left(F_{1}\right)$ and $h\left(f_{2}\right)=h\left(F_{2}\right)$. Now the coefficients of $l_{1}^{\prime}, \ldots, l_{t}^{\prime}, m_{1}^{\prime}, \ldots, m_{t}^{\prime}$ are rational functions of the zeros of $f_{1}$ and $f_{2}$. Hence by (4.7) and (4.5),

$$
h\left(l_{i}^{\prime}\right) \leq c_{74}, \quad h\left(m_{i}^{\prime}\right) \leq c_{74} \quad \text { for } i=1, \ldots, t .
$$

Choose $a \in O_{S} \backslash\{0\}$ such that $h(a) \leq c_{75}$ and

$$
a l_{i}^{\prime}, a m_{i}^{\prime} \in O_{T}\left[X_{1}, \ldots, X_{n}\right] \quad \text { for } i=1, \ldots, t .
$$

Then the linear forms $l_{i}:=a l_{i}^{\prime}, m_{i}:=a m_{i}^{\prime}(i=1, \ldots, t)$ satisfy (7.5.a,b,c).

By (7.5.b,c), (1.5) and (4.6), we have

$$
m_{T}\left(\lambda_{i}\right) \leq c_{76}, \quad \sigma\left(\lambda_{i}\right)=\lambda_{\sigma(i)} \quad \text { for } i=1, \ldots, t, \sigma \in \operatorname{Gal}(L / K) .
$$

Let $\mathcal{G}$ be the hypergraph with vertices $1, \ldots, t$ whose edges are those subsets $I$ of $\{1, \ldots, t\}$ for which $\left\{l_{i}: i \in I\right\}$ is linearly dependent and each proper, non-empty subset of $\left\{l_{i}: i \in I\right\}$ is linearly independent. The hypergraph corresponding to $m_{1}, \ldots, m_{t}$ is exactly the same, by (7.5.b). Let $\mathcal{G}_{1}, \ldots, \mathcal{G}_{v}$ denote the connected components of $\mathcal{G}$ (two vertices belong to the same 
connected component if and only if there is a path connecting them). By (6.8), there are uniquely determined numbers $c_{i j}^{(I)}, d_{i j}^{(I)} \in L^{*}$ such that

$$
l_{j}=-\sum_{i \in I \backslash\{j\}} c_{i j}^{(I)} l_{i} \quad \text { and } \quad m_{j}=-\sum_{i \in I \backslash\{j\}} d_{i j}^{(I)} m_{i}
$$

for each edge $I$ of $\mathcal{G}$ and each $j \in I$. By (6.10) and (7.5.a) we have

$$
h\left(c_{i j}^{(I)}\right), h\left(d_{i j}^{(I)}\right) \leq c_{77} .
$$

From (7.5.b) and (7.7) it follows that

$$
m_{j}=-\sum_{i \in I \backslash\{j\}} c_{i j}^{(I)}\left(\lambda_{i} / \lambda_{j}\right) m_{i} .
$$

Hence

$$
\lambda_{i} / \lambda_{j}=d_{i j}^{(I)} / c_{i j}^{(I)}
$$

Together with (7.8) this implies the following: if $i, j$ belong to the same connected component then

$$
h\left(\lambda_{i} / \lambda_{j}\right) \leq c_{78} .
$$

We assume that $\left\{l_{1}, \ldots, l_{n}\right\}$ and hence $\left\{m_{1}, \ldots, m_{n}\right\}$ is linearly independent, which is no restriction. Put $\Delta=\operatorname{det}\left(l_{1}, \ldots, l_{n}\right)$. By assumption, $\mathfrak{M}=$ $\left(\mathbf{e}_{1}, \ldots, \mathbf{e}_{n-1}, \alpha \mathbf{e}_{n}, \beta \mathbf{e}_{n}\right)$, and by (2.3) and (4.6), the ideal $\mathfrak{a}=(\alpha, \beta)$ has $|\mathfrak{a}|_{S} \leq c_{79}$. Let $h$ be the cardinality of the unit group of the residue class ring $O_{T} / \Delta \mathfrak{a}$. Then, by (7.5.a), (4.6) and $|\mathfrak{a}|_{S} \leq c_{79}$, we have

$$
h \leq c_{80} .
$$

Let $A_{j}=\left\{\lambda_{i}: i \in \mathcal{G}_{j}\right\}$ for $j=1, \ldots, v$. Each $\sigma$ maps linearly (in)dependent linear forms onto linearly (in)dependent linear forms, hence there is a permutation $\sigma^{*}$ of $1, \ldots, v$ such that $\sigma\left(\mathcal{G}_{j}\right)=\mathcal{G}_{\sigma^{*}(j)}$ for $j=1, \ldots, v$. Therefore, by (7.6) and (7.5.c), $\sigma\left(A_{j}\right)=A_{\sigma^{*}(j)}$ for $j=1, \ldots, v$ and $\sigma \in \operatorname{Gal}(L / K)$. Further, (7.9) holds. Hence we can apply Lemma 7 with $v$ instead of $t$ and we infer that there are $\eta_{1}, \ldots, \eta_{v} \in O_{T}^{*}$ such that

$$
\sigma\left(\eta_{j}\right)=\eta_{\sigma^{*}(j)} \quad \text { for } j=1, \ldots, v \text { and } \sigma \in \operatorname{Gal}(L / K)
$$

and

$$
h\left(\eta_{j}^{h} \lambda_{i}\right) \leq c_{81} \quad \text { for } \lambda_{i} \in A_{j} .
$$

We note that in order to use Lemma 7 we must have an estimate $\left|D_{L}\right| \leq c_{82}$ where $D_{L}$ is the discriminant of the splitting field $L$ of $F_{1}$ and $F_{2}$ over $K$. However, this can be done by using Example 4 from Section 1, (7.5.a) and Lemma 17. 
For each $i \in\{1, \ldots, t\}$, put $\varepsilon_{i}=\eta_{j}$ if $i \in \mathcal{G}_{j}$ for $j=1, \ldots, v$. Then

$$
\begin{cases}\varepsilon_{i}=\varepsilon_{k} & \text { if } i, k \in \mathcal{G}_{j} \text { for some } j \in\{1, \ldots, v\} \\ \sigma\left(\varepsilon_{i}\right)=\varepsilon_{\sigma(i)} & \text { for } i=1, \ldots, t \text { and for all } \sigma \in \operatorname{Gal}(L / K) \\ h\left(\varepsilon_{i}^{h} \lambda_{i}\right) \leq c_{81} & \text { for } i=1, \ldots, t\end{cases}
$$

We claim that there is a matrix $U^{\prime \prime} \in G(n, \mathfrak{a})$ such that

$$
\varepsilon_{i}^{h} l_{i}(\mathbf{X})=l_{i}\left(U^{\prime \prime} \mathbf{X}\right) \quad \text { for } i=1, \ldots, t .
$$

Let $B \in O_{T}^{n, n}$ be the matrix whose $i$ th row consists of the coefficients of $l_{i}$, and $B^{\prime} \in O_{T}^{n, n}$ the matrix whose $i$ th row consists of the coefficients of $\varepsilon_{i}^{h} l_{i}$ for $i=1, \ldots, n$. By our choice of $h$ we have $\varepsilon_{i}^{h} \equiv 1 \bmod \Delta \mathfrak{a}$, hence $B^{\prime} \equiv B \bmod \Delta \mathfrak{a}\left(\right.$ in $\left.O_{T}\right)$. Further, $\left(\operatorname{det} B^{\prime}\right) /(\operatorname{det} B)=\left(\varepsilon_{1} \ldots \varepsilon_{n}\right)^{h} \in O_{T}^{*}$. Hence by Lemma 10 with $K$ replaced by $L$, there is a matrix $U^{\prime \prime} \in O_{T}^{n, n}$ such that $\operatorname{det} U^{\prime \prime} \in O_{T}^{*}$,

$$
U^{\prime \prime} \equiv\left(\begin{array}{ccc}
\zeta_{1} & & 0 \\
& \ddots & \\
0 & & \zeta_{n}
\end{array}\right) \bmod \mathfrak{a} \quad \text { with some } \zeta_{1}, \ldots, \zeta_{n} \in O_{T}^{*}
$$

and

$$
B^{\prime}=B U^{\prime \prime} .
$$

This matrix $U^{\prime \prime}$ satisfies (7.12) for $i=1, \ldots, n$. For every $i \in\{n+1, \ldots, t\}$ there is a unique subset $I_{i}$ of $\{1, \ldots, n\}$ such that $l_{i}=\sum_{j \in I_{i}} c_{j} l_{j}$ for certain $c_{j} \in L^{*}$. Now $I_{i} \cup\{i\}$ is an edge of $\mathcal{G}$, hence by (7.11), $\varepsilon_{j}=\varepsilon_{i}$ for $j \in I_{i}$. Therefore,

$$
\varepsilon_{i}^{h} l_{i}=\sum_{j \in I_{i}} c_{j}\left(\varepsilon_{j}^{h} l_{j}\right) \quad \text { for } j=n+1, \ldots, t .
$$

We conclude that (7.12) holds also for $i=n+1, \ldots, t$. By (7.11) and (7.5.c) we have

$$
\sigma\left(\varepsilon_{i}^{h} l_{i}\right)=\varepsilon_{\sigma(i)}^{h} l_{\sigma(i)} \quad \text { for } i=1, \ldots, t \text { and for each } \sigma \in \operatorname{Gal}(L / K) .
$$

Thus the matrix $U^{\prime \prime}$ satisfying (7.12) must have its entries in $K$. Together with (7.13) this implies that $U^{\prime \prime} \in G(n, \mathfrak{a})$, whence, by $G(n, \mathfrak{a}) \subseteq G(\mathfrak{M})$, $U^{\prime \prime} \in G(\mathfrak{M})$ holds.

Put $\lambda_{i}^{\prime}=\varepsilon_{i}^{h} \lambda_{i}$ for $i=1, \ldots, t$. Then, by (7.5.b) and (7.12), there is a matrix $U^{\prime} \in G(\mathfrak{M})$ such that

$$
\lambda_{i}^{\prime} m_{i}(\mathbf{X})=l_{i}\left(U^{\prime} \mathbf{X}\right) \quad \text { for } i=1, \ldots, t .
$$

By (7.11) we have $h\left(\lambda_{i}^{\prime}\right) \leq c_{81}$, and by (7.5.a), $h\left(l_{i}\right) \leq c_{73}$, and $h\left(m_{i}\right) \leq c_{73}$ for $i=1, \ldots, t$. This implies that $h\left(U^{\prime}\right) \leq c_{72}$. Further, by (7.5.c), (7.6) and (7.11), we get $\prod_{i=1}^{t}\left(\lambda_{i}^{\prime}\right)^{k_{i}} \in K^{*}$. Hence there is a $\lambda^{\prime} \in K^{*}$ such that $F_{2}(\mathbf{X})=\lambda^{\prime} F_{1}\left(U^{\prime} \mathbf{X}\right)$, which proves Lemma 18. 
Proof of Corollary 3. Immediate consequence of (1.12) and Corollary 1 with $D(\mathfrak{M}, F)=(1)$.

In the proofs of Corollaries 4 and $5, c_{82}, \ldots, c_{93}$ will denote effectively computable numbers of the form $c^{\prime}|\mathfrak{d}|_{S}^{c^{\prime \prime}}$, where $c^{\prime}, c^{\prime \prime}$ depend only on $d,\left|D_{L}\right|$, $s, P, n$ and $r$.

Proof of Corollary 4. By Theorem $2,(\mathfrak{M}, F)$ is weakly equivalent to a pair $\left(\mathfrak{M}^{\prime}, F^{\prime \prime}\right)$, where $\mathfrak{M}^{\prime}$ is a reduced $O_{S^{-}}$-lattice and $h\left(F^{\prime \prime}\right) \leq c_{82}$. Choose $\lambda \in K^{*}$ and an isomorphism $\varphi: \mathfrak{M} \rightarrow \mathfrak{M}^{\prime}$ such that $\lambda F^{\prime \prime}(\mathbf{x})=$ $F(\varphi(\mathbf{x}))$ for $\mathbf{x} \in \mathfrak{M}$. Then $(\lambda)=\mathfrak{c}(\mathfrak{M}, F) \mathfrak{c}\left(\mathfrak{M}^{\prime}, F^{\prime \prime}\right)^{-1}$. By using some arguments from the proof of Lemma 18 one can show that $F^{\prime \prime}(\mathbf{X})$ has a factorization $F^{\prime \prime}(\mathbf{X})=\lambda \prod_{i=1}^{t} l_{i}(\mathbf{X})^{k_{i}}$ where the $l_{i}$ are pairwise non-proportional linear forms with $l_{i}(\mathbf{X}) \in O_{T}\left[X_{1}, \ldots, X_{n}\right], h\left(l_{i}\right) \leq c_{83}$ for $i=1, \ldots, t$ and $h(\lambda) \leq c_{84}$. Hence $m_{S}\left(\mathfrak{c}\left(\mathfrak{M}^{\prime}, F^{\prime \prime}\right)\right) \leq c_{85}$ and so

$$
m_{S}(\lambda) \leq m_{S}(\mathfrak{c}(\mathfrak{M}, F)) m_{S}\left(\mathfrak{c}\left(\mathfrak{M}^{\prime}, F^{\prime \prime}\right)\right) \leq c_{86} m_{S}(\mathfrak{c}(\mathfrak{M}, F)) .
$$

By Lemma 6 , we can choose $\varepsilon \in O_{S}^{*}$ such that

$$
h\left(\varepsilon^{r} \lambda\right) \leq c_{87} m_{S}(\mathfrak{c}(\mathfrak{M}, F)) .
$$

We note that in Lemma $6,\left|D_{K}\right|$ was involved, but $\left|D_{K}\right| \leq\left|D_{L}\right|$. Put $F^{\prime}=$ $\varepsilon^{r} \lambda F^{\prime \prime}$. Since the mapping $\mathbf{x} \mapsto \varepsilon \varphi(\mathbf{x})$ is an isomorphism $\mathfrak{M} \rightarrow \mathfrak{M}^{\prime}$, the pairs $(\mathfrak{M}, F)$ and $\left(\mathfrak{M}^{\prime}, F^{\prime}\right)$ are equivalent. Further, by $(7.14)$ and $\mathfrak{c}(\mathfrak{M}, F)=\mathfrak{c}$,

$$
h\left(F^{\prime}\right) \leq c_{88} m_{S}(\mathfrak{c}) .
$$

This proves the first inequality of Corollary 4. The second one follows by applying Lemma 17.

Proof of Corollary 5. By Corollary 4, there are a reduced $O_{S^{-}}$ lattice $\mathfrak{M}^{\prime}$ of rank $n$ and a decomposable form $F^{\prime}$ on $K^{n}$ such that $(\mathfrak{M}, F)$ and $\left(\mathfrak{M}^{\prime}, F^{\prime}\right)$ are equivalent and $h\left(F^{\prime}\right) \leq c_{89} m_{S}(\mathfrak{c})$. This implies

$$
h\left(F^{\prime}\left(\mathbf{e}_{j}\right)\right) \leq c_{89} m_{S}(\mathfrak{c}) \quad \text { for } j=1, \ldots, n .
$$

Further, by (2.3) we have either (i) $\mathfrak{M}^{\prime}=\left(\mathbf{e}_{1}, \ldots, \mathbf{e}_{n}\right)$ where $\mathfrak{M}^{\prime}$ is free, or (ii) $\mathfrak{M}^{\prime}=\left(\mathbf{e}_{1}, \ldots, \mathbf{e}_{n-1}, \alpha \mathbf{e}_{n}, \beta \mathbf{e}_{n}\right)$ with $\alpha, \beta \in O_{S} \backslash\{0\}$ satisfying (2.3). By a well-known argument (see e.g. the proof of Lemma 1 in [2], Ch. II, §1) it follows that there are $u_{21}, \ldots, u_{n 1}$ with $F^{\prime}\left(\mathbf{e}_{1}+u_{21} \mathbf{e}_{2}+\ldots+u_{n 1} \mathbf{e}_{n}\right) \neq 0$ such that $u_{j 1} \in\{0,1, \ldots, r\}$ in case (i) and $u_{j 1} \in\{0, \alpha, 2 \alpha, \ldots, r \alpha\}$ in case (ii) for $j=2, \ldots, n$. Put $\mathbf{e}_{1}^{\prime}:=\mathbf{e}_{1}+\sum_{i=2}^{n} u_{i 1} \mathbf{e}_{i}$. We can inductively construct $\mathbf{e}_{2}^{\prime}, \ldots, \mathbf{e}_{n}^{\prime}$ such that $F^{\prime}\left(\mathbf{e}_{j}^{\prime}\right) \neq 0$ and that

$$
\mathbf{e}_{j}^{\prime}=u_{1 j} \mathbf{e}_{1}^{\prime}+\ldots+u_{j-1, j} \mathbf{e}_{j-1}^{\prime}+\mathbf{e}_{j}+u_{j+1, j} \mathbf{e}_{j+1}+\ldots+u_{n, j} \mathbf{e}_{n}
$$

with $u_{i j} \in\{0,1, \ldots, r\}$ in case (i) and $u_{i j} \in\{0, \alpha, 2 \alpha, \ldots, r \alpha\}$ in case (ii) for $i=2, \ldots, n, j=2, \ldots, n$. It is easy to check that $\mathfrak{M}^{\prime}=\left(\mathbf{e}_{1}^{\prime}, \ldots, \mathbf{e}_{n}^{\prime}\right)$ in case (i) and $\mathfrak{M}^{\prime}=\left(\mathbf{e}_{1}^{\prime}, \ldots, \mathbf{e}_{n-1}^{\prime}, \alpha \mathbf{e}_{n}^{\prime}, \beta \mathbf{e}_{n}^{\prime}\right)$ in case (ii). Let $V=\left(v_{i j}\right)$ be the 
$n \times n$ matrix defined by $\mathbf{e}_{i}^{\prime}=V \mathbf{e}_{i}$ for $i=1, \ldots, n$ and put $F^{\prime \prime}(\mathbf{X})=F^{\prime}(V \mathbf{X})$. Then $\left(\mathfrak{M}^{\prime}, F^{\prime \prime}\right)$ is equivalent to $\left(\mathfrak{M}^{\prime}, F^{\prime}\right)$ and hence to $(\mathfrak{M}, F)$. Further, it is easy to see that $h(V) \leq c_{90}$. Hence we get $h\left(F^{\prime \prime}\right) \leq c_{91} m_{S}(\mathfrak{c})$, which implies that

$$
h\left(F^{\prime \prime}\left(\mathbf{e}_{j}\right)\right) \leq c_{91} m_{S}(\mathfrak{c}) \quad \text { for } j=1, \ldots, n .
$$

Further, $F^{\prime \prime}\left(\mathbf{e}_{1}\right) \ldots F^{\prime \prime}\left(\mathbf{e}_{n}\right)=F^{\prime}\left(\mathbf{e}_{1}^{\prime}\right) \ldots F^{\prime}\left(\mathbf{e}_{n}^{\prime}\right) \neq 0$.

There is an $O_{S}$-module isomorphism $\varphi: \mathfrak{M}^{\prime} \rightarrow \mathfrak{M}$ such that $F^{\prime \prime}(\mathbf{x})=$ $F(\varphi(\mathbf{x}))$ for each $\mathbf{x} \in \mathfrak{M}^{\prime}$. In case (i) we put $\omega_{j}=\varphi\left(\mathbf{e}_{j}\right)$ for $j=1, \ldots, n$, while in case (ii) we put $\omega_{j}=\varphi\left(\mathbf{e}_{j}\right)$ for $j=1, \ldots, n-1, \omega_{n}=\varphi\left(\alpha \mathbf{e}_{n}\right)$, $\omega_{n+1}=\varphi\left(\beta \mathbf{e}_{n}\right)=\gamma \omega_{n}$ where $\gamma=\beta / \alpha$ and, by $(2.3), h(\gamma) \leq c_{92}$ with some effectively computable $c_{92}$ which depends only on $d$ and $\left|D_{K}\right|$. Therefore $\mathfrak{M}=\left(\omega_{1}, \ldots, \omega_{n}\right)$ where either $\mathfrak{M}$ is free and $m=n$, or $\mathfrak{M}$ is not free, $m=n+1$ and $\omega_{n+1}=\gamma \omega_{n}$ with the above $\gamma, F\left(\omega_{1}\right) \ldots F\left(\omega_{m}\right) \neq 0$, and, by (7.16) and (2.3), we get in both cases

$$
h\left(F\left(\omega_{j}\right)\right) \leq c_{93} \quad \text { for } j=1, \ldots, m .
$$

This proves the first inequality of (2.4). The second one follows by applying Lemma 17.

Proof of Corollary 6 . If there are $\mu \in M^{*}$ and a subfield $M^{\prime}$ of $M$ such that $\mu \mathfrak{M} \subseteq M^{\prime}$, then

$$
N_{M / K}(\alpha)=N_{M / K}(\mu)^{-1} N_{M^{\prime} / K}(\mu \alpha)^{\left[M: M^{\prime}\right]} \quad \text { for all } \alpha \in \mathfrak{M} .
$$

Hence $\mathfrak{D}\left(\mathfrak{M}, N_{M / K}\right)=\mathfrak{D}\left(\mu \mathfrak{M}, N_{M^{\prime} / K}\right)$. Therefore we may, and shall, assume that there is no $\mu \in M^{*}$ such that $\mu \mathfrak{M}$ is contained in a proper subfield of $M$. Note that in this case, the normal closure $L$ of $M / K$ is the splitting field of the restriction of $N_{M / K}$ to $K \mathfrak{M}$.

Next $c_{94}, c_{95}, c_{96}$ will denote effectively computable numbers of the form $\left.c^{\prime}|\mathfrak{d}|\right|_{S} ^{\prime \prime}$ where $\mathfrak{d}=\mathfrak{D}(\mathfrak{M})$ and $c^{\prime}, c^{\prime \prime}$ depend only on $d, r=[M: K],\left|D_{L}\right|, s$, $P$ and $n=\operatorname{rank} \mathfrak{M}$. By Theorem 2, there are a reduced $O_{S^{-}}$-lattice $\mathfrak{M}^{\prime \prime}$ of rank $n$, a decomposable form $F^{\prime}$ on $K^{n}$, an isomorphism $\varphi: \mathfrak{M}^{\prime \prime} \rightarrow \mathfrak{M}$ and $\lambda \in K^{*}$ such that

$$
F^{\prime}(\mathbf{X})=\lambda N_{M / K}(\varphi(\mathbf{x})) \quad \text { for all } \mathbf{x} \in \mathfrak{M}^{\prime \prime} \text { and } h(F) \leq c_{94} .
$$

$\varphi$ can be extended uniquely to a $K$-linear mapping $K^{n} \rightarrow M$. Denote the conjugates of $\alpha \in M$ over $K$ by $\alpha=\alpha^{(1)}, \ldots, \alpha^{(r)}$ and define the linear functions $l_{j}: K^{n} \rightarrow L(j=1, \ldots, r)$ by

$$
l_{j}(\mathbf{x})=\left\{\varphi\left(\mathbf{e}_{1}\right)^{-1} \varphi(\mathbf{x})\right\}^{(j)} \quad \text { for all } \mathbf{x} \in K^{n} .
$$

Then, by (7.17),

$$
l_{1}(\mathbf{x}) \ldots l_{r}(\mathbf{x})=N_{M / K}\left(\varphi\left(\mathbf{e}_{1}\right)\right)^{-1} N_{M / K}(\varphi(\mathbf{x}))=F^{\prime}\left(\mathbf{e}_{1}\right)^{-1} F^{\prime}(\mathbf{x})=: G(\mathbf{x}) .
$$


Now (7.17) implies that

$$
h(G) \leq c_{95} \quad \text { with } c_{95}=c_{94}^{2} .
$$

By a similar argument to the proof of Lemma 18, and using the fact that each $l_{i}$ is a linear form one of whose coefficients is equal to 1 , it follows that

$$
h\left(l_{j}\right) \leq c_{96} \quad \text { for } j=1, \ldots, r .
$$

Let $\mathfrak{M}^{\prime}=\varphi\left(\mathbf{e}_{1}\right)^{-1} \mathfrak{M}$. If $\mathfrak{M}^{\prime \prime}=O_{S}^{n}$ then take $\omega_{i}=l_{1}\left(\mathbf{e}_{i}\right)$ for $i=1, \ldots, n$, and if $\mathfrak{M}^{\prime \prime}=\left(\mathbf{e}_{1}, \ldots, \mathbf{e}_{n-1}, \alpha \mathbf{e}_{n}, \beta \mathbf{e}_{n}\right)$ with $\alpha, \beta \in O_{S}$ satisfying (2.3), then take $\omega_{i}=l_{1}\left(\mathbf{e}_{i}\right)$ for $i=1, \ldots, n-1, \omega_{n}=\alpha l_{1}\left(\mathbf{e}_{n}\right)$ and $\omega_{n+1}=\beta l_{1}\left(\mathbf{e}_{n}\right)=\gamma \omega_{n}$ with $\gamma=\beta / \alpha$. Put $m=n$ in the first case and $m=n+1$ in the second case. Then $\mathfrak{M}^{\prime}$ is similar to $\mathfrak{M}$ and we have $\mathfrak{M}^{\prime}=\left(\omega_{1}, \ldots, \omega_{n}\right)$. Further, as we have seen in the proof of Lemma $17,\left|D_{L}\right|$ can be estimated from above in terms of $\left|D_{M}\right|$ and $[M: K]$ only. Hence, by (7.18) and by (2.3) in the second case, we get the first inequality of (2.5). The second one follows by using Lemma 17.

Acknowledgements. We thank the referee for his helpful remarks.

\section{References}

[1] B. J. Birch and J. R. Merriman, Finiteness theorems for binary forms with given discriminant, Proc. London Math. Soc. 25 (1972), 385-394.

[2] Z. I. Borevich and I. R. Shafarevich, Number Theory, 2nd ed., Academic Press, New York and London 1967.

[3] J. H. Evertse, Decomposable form equations with a small linear scattering, to appear.

[4] J. H. Evertse and K. Györy, Decomposable form equations, in: New Advances in Transcendence Theory, A. Baker (ed.), Cambridge University Press, 1988, 175-202.

[5] - , -, Thue-Mahler equations with a small number of solutions, J. Reine Angew. Math. 399 (1989), 60-80.

[6] - - - Effective finiteness results for binary forms with given discriminant, Compositio Math. 79 (1991), 169-204.

[7] J. H. Evertse, K. Győry, C. L. Stewart and R. Tijdeman, S-unit equations and their applications, in: New Advances in Transcendence Theory, A. Baker (ed.), Cambridge University Press, 1988, 110-174.

[8] K. Győry, Sur les polynômes à coefficients entiers et de discriminant donné, Acta Arith. 23 (1973), 419-426.

[9] -, On polynomials with integer coefficients and given discriminant, $V, \mathfrak{p}$-adic generalizations, Acta Math. Acad. Sci. Hungar. 32 (1978), 175-190.

[10] - On the number of solutions of linear equations in units of an algebraic number field, Comment. Math. Helv. 54 (1979), 583-600.

[11] - On S-integral solutions of norm form, discriminant form and index form equations, Studia Sci. Math. Hungar. 16 (1981), 149-161. 
[12] K. Györy, Effective finiteness theorems for polynomials with given discriminant and integral elements with given discriminant over finitely generated domains, J. Reine Angew. Math. 346 (1984), 54-100.

[13] G. J. Janusz, Algebraic Number Fields, Academic Press, New York and London 1973.

[14] I. Kaplansky, Modules over Dedekind rings and valuation rings, Trans. Amer. Math. Soc. 72 (1952), 327-340.

[15] S. Lang, Algebraic Number Theory, Springer, 1970.

[16] K. Mahler, Über die Annäherung algebraischer Zahlen durch periodische Algorithmen, Acta Math. 68 (1937), 109-144.

[17] T. Nagell, Contributions à la théorie des modules et des anneaux algébriques, Ark. Mat. 6 (1965), 161-178.

[18] W. Narkiewicz, Elementary and Analytic Theory of Algebraic Numbers, Polish Scientific Publishers, Warszawa 1974.

[19] H. M. Stark, Some effective cases of the Brauer-Siegel theorem, Invent. Math. 23 (1974), 135-152.

[20] O. Zariski and P. Samuel, Commutative Algebra, Vol. I, D. Van Nostrand Co., Toronto-New York-London 1958.

DEPARTMENT OF MATHEMATICS

AND COMPUTER SCIENCE

UNIVERSITY OF LEIDEN

P.O. BOX 9512

2300 RA LEIDEN, THE NETHERLANDS
MATHEMATICAL INSTITUTE LAJOS KOSSUTH UNIVERSITY H-4010 DEBRECEN, HUNGARY

Received on 21.8.1990

and in revised form on 13.3.1991 